Florida International University FIU Digital Commons

\title{
The HI Chronicles of LITTLE THINGS Blue Compact Dwarf Galaxies
}

Trisha L. Ashley

trisha.ashley@gmail.com

DOI: $10.25148 /$ etd.FI14071102

Follow this and additional works at: https://digitalcommons.fiu.edu/etd

\section{Recommended Citation}

Ashley, Trisha L., "The HI Chronicles of LITTLE THINGS Blue Compact Dwarf Galaxies" (2014). FIU Electronic Theses and Dissertations. 1441.

https://digitalcommons.fiu.edu/etd/1441 


\title{
FLORIDA INTERNATIONAL UNIVERSITY
}

\author{
Miami, Florida
}

THE H I CHRONICLES OF LITTLE THINGS BLUE COMPACT DWARF GALAXIES

A dissertation submitted in partial fulfillment of the

requirements for the degree of

DOCTOR OF PHILOSOPHY

in

PHYSICS

by

Trisha Lynn Ashley

2014 
To: Interim Dean Michael R. Heithaus

College of Arts and Sciences

This dissertation, written by Trisha Lynn Ashley, and entitled The H I Chronicles of LITTLE THINGS Blue Compact Dwarf Galaxies, having been approved in respect to style and intellectual content, is referred to you for judgement.

We have read this dissertation and recommend that it be approved.

Deidre Hunter

$\begin{array}{r}\hline \text { James Webb } \\ \hline \text { Rosemary Hickey-Vargas } \\ \hline \text { Walter Van Hamme }\end{array}$

Caroline Simpson, Major Professor

Date of Defense: May 27, 2014

The dissertation of Trisha Lynn Ashley is approved.

Interim Dean Michael R. Heithaus

College of Arts and Sciences

Dean Lakshmi N. Reddi

University Graduate School

Florida International University, 2014 
(C) Copyright 2014 by Trisha Lynn Ashley

All rights reserved. 


\section{ACKNOWLEDGMENTS}

I would first like to acknowledge the LITTLE THINGS team, without their hard work, advice, and dedication to science this dissertation would not have been possible and I would not be the scientist that I am today. Specifically I would like to recognize Megan Johnson, who allowed me to use some of her GBT data in this dissertation, has been a great help in teaching me about the GBT data, and suggested that I search the environments of BCDs for nearby companions. I would also like to single out LITTLE THINGS team member Bruce Elmegreen, who has helped me expand my research knowledge greatly by always providing thoughtful comments. I would also like to acknowledge the LITTLE THINGS team's fearless leader and my committee member, Deidre Hunter; she always has the right answers and the best advice on my research. I would also like to thank my adviser, Caroline Simpson, for her help, sending me to amazing conferences, helping me advance my career, and overall being a wonderful mentor. I would also like to thank my committee members for putting in the time and hard work of getting through my dissertation and listening to my talks.

I would like to also acknowledge my friends and fellow graduate students at FIU for their support, advice, and help. Ramona Perez, Sarah Dhalla, Nauraj Pokhrel, Yuehai (and Lian) Yang, Gopal Bhatta, and Mr. Scrunchies, you have all helped me improve my research talks, kept me mostly sane through the rough spots in graduate school, and have been amazing friends overall. Without you, I probably would have cracked long ago. I would also like to acknowledge coffee, chocolate, candy, and donuts; you have helped me get through it all and without you I would not have been able to complete this dissertation.

This project was funded in part through the FIU Dissertation Year Fellowship. This project was funded in part by the National Science Foundation under grant Nos. AST0707563 AST-0707426, AST-0707468, and AST 0707835 to Deidre A. Hunter, Bruce G. Elmegreen, Caroline E. Simpson, and Lisa M. Young. The GALEX work was funded 
by NASA through grant NNX07AJ36G and by cost-sharing from Lowell Observatory. This research has made use of the NASA/IPAC Extragalactic Database (NED) which is operated by the Jet Propulsion Laboratory, California Institute of Technology, under contract with the National Aeronautics and Space Administration (NASA). 


\section{ABSTRACT OF THE DISSERTATION \\ THE H I CHRONICLES OF LITTLE THINGS BLUE COMPACT DWARF GALAXIES by}

Trisha Lynn Ashley

Florida International University, 2014

Miami, Florida

Professor Caroline Simpson, Major Professor

Star formation occurs when the gas (mostly atomic hydrogen; H I) in a galaxy becomes disturbed, forming regions of high density gas, which then collapses to form stars. In dwarf galaxies it is still uncertain which processes contribute to star formation and how much they contribute to star formation. Blue compact dwarf (BCD) galaxies are low mass, low shear, gas rich galaxies that have high star formation rates when compared to other dwarf galaxies. What triggers the dense burst of star formation in BCDs but not other dwarfs is not well understood. It is often suggested that BCDs may have their starburst triggered by gravitational interactions with other galaxies, dwarf-dwarf galaxy mergers, or consumption of intergalactic gas. However, there are BCDs that appear isolated with respect to other galaxies, making an external disturbance unlikely.

Here, I study six apparently isolated BCDs from the LITTLE THINGS ${ }^{1}$ sample in an attempt to understand what has triggered their burst of star formation. LITTLE THINGS is an H I survey of 41 dwarf galaxies. Each galaxy has high angular and velocity resolution H I data from the Very Large Array (VLA) telescope and ancillary stellar data. I use these data to study the detailed morphology and kinematics of each galaxy, looking for signatures of starburst triggers. In addition to the VLA data, I have collected Green Bank Telescope data for the six BCDs. These high sensitivity, low resolution data are used to

\footnotetext{
${ }^{1}$ Local Irregulars That Trace Luminosity Extremes, The H I Nearby Galaxy Survey; https://science.nrao.edu/science/surveys/littlethings
} 
search the surrounding area of each galaxy for extended emission and possible nearby companion galaxies.

The VLA data show evidence that each BCD has likely experienced some form of external disturbance despite their apparent isolation. These external disturbances potentially seen in the sample include: ongoing/advanced dwarf-dwarf mergers, an interaction with an unknown external object, and external gas consumption. The GBT data result in no nearby, separate $\mathrm{H}$ I companions at the sensitivity of the data. These data therefore suggest that even though these BCDs appear isolated, they have not been evolving in isolation. It is possible that these external disturbances may have triggered the starbursts that defines them as BCDs. 


\section{TABLE OF CONTENTS}

CHAPTER

PAGE

1 Introduction 1

2 Sample 5

2.1 Haro $29 \ldots \ldots \ldots \ldots \ldots \ldots \ldots$

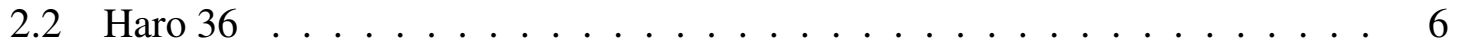

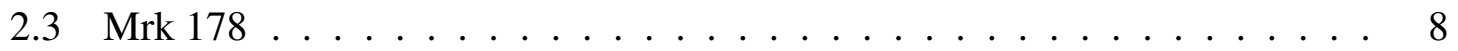

2.4 VII Zw $403 \ldots \ldots \ldots \ldots$

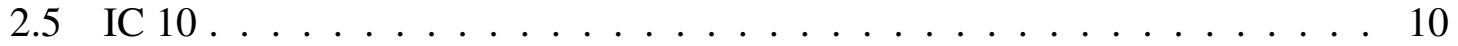

2.6 NGC $3738 \ldots \ldots \ldots \ldots \ldots \ldots$

3 Observations and Data Reduction 13

3.1 The Very Large Array Telescope . . . . . . . . . . . . . . . . . . . . 13

3.2 The Green Bank Telescope . . . . . . . . . . . . . . . . . . . . . 18

4 Results: Haro $29 \quad 25$

4.1 Stellar Component . . . . . . . . . . . . . . . . 25

4.2 VLA H I Morphology . . . . . . . . . . . . . . . . . . . . 25

4.3 VLA H I Velocity and Velocity Dispersion Field . . . . . . . . . . . . . . . 28

4.4 GBT H I Morphology and Velocity Field . . . . . . . . . . . . . . . 28

4.5 H I Mass . . . . . . . . . . . . . . . . . . . . . . . . . . . . . . . . 29

5 Discussion: Haro 29

6 Results: Haro $36 \quad 35$

6.1 Stellar Component . . . . . . . . . . . . . . . . . 35

6.2 VLA H I Morphology . . . . . . . . . . . . . . . . . . . . 35

6.3 VLA H I Velocity and Velocity Dispersion Field . . . . . . . . . . . . . . 37

6.4 GBT H I Morphology and Velocity Field . . . . . . . . . . . . . . . . 38

6.5 H I Mass . . . . . . . . . . . . . . . . . . . . . . . . . . . . 38

7 Discussion: Haro 36

8 Results: Mrk $178 \quad 49$

8.1 Stellar Component . . . . . . . . . . . . . . . . . . . . . . . . . . . . 49

8.2 VLA H I Morphology . . . . . . . . . . . . . . . . . . . . . . . . . . . . . . . . . 50

8.3 VLA H I Velocity and Velocity Dispersion Field . . . . . . . . . . . . . 52

8.4 GBT H I Morphology and Velocity Field . . . . . . . . . . . . . . . . . 52

8.5 H I Mass . . . . . . . . . . . . . . . . . . . . . . . 53

9 Discussion: Mrk 178 
10 Results: VII Zw 403

10.1 Stellar Component . . . . . . . . . . . . . . . . . 61

10.2 VLA H I Morphology . . . . . . . . . . . . . . . . . . . . . 61 . . . . . . . . 62

10.3 VLA H I Velocity and Velocity Dispersion Field . . . . . . . . . . . . 62

10.4 GBT H I Morphology and Velocity Field . . . . . . . . . . . . . . . 62

10.5 H I Mass . . . . . . . . . . . . . . . . . . . . . . 664

11 Discussion: VII Zw 403

12 Results: IC 10

12.1 Stellar Component . . . . . . . . . . . . . . . . . 76

12.2 VLA H I Morphology . . . . . . . . . . . . . . . . . . 76

12.3 VLA H I Velocity and Velocity Dispersion Field . . . . . . . . . . . 77

12.4 GBT H I Morphology and Velocity Field . . . . . . . . . . . . . . 78

12.5 H I Mass . . . . . . . . . . . . . . . . . . . . . . . . 81

13 Discussion: IC $10 \quad 81$

13.1 A Galaxy Still in Formation . . . . . . . . . . . . . . . . . . 82

13.2 A Galaxy with a Northern Bridge . . . . . . . . . . . . . . . . . . . . . . . . . . 83

13.3 An Advanced Merger . . . . . . . . . . . . . . . . . . . . . . 84

14 Results: NGC $3738 \quad 93$

14.1 Stellar Component . . . . . . . . . . . . . . . . . 93

14.2 VLA H I Morphology . . . . . . . . . . . . . . . . . . . . . . . . . . . . . . . . . . . . . . . .

14.3 VLA H I Velocity and Velocity Dispersion Field . . . . . . . . . . . . . . . . . . . . 94

14.4 GBT H I Morphology and Velocity Field . . . . . . . . . . . . . . . 95

14.5 H I Mass . . . . . . . . . . . . . . . . . . . . . . . . 96

15 Discussion: NGC 3738

$\begin{array}{ll}16 \text { Conclusion } & 100\end{array}$

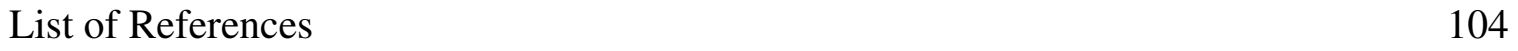

$\begin{array}{ll}\text { Appendices } & 109\end{array}$

$\begin{array}{ll}\text { Vita } & 122\end{array}$ 


\section{LIST OF TABLES}

\section{TABLE}

PAGE

1 Basic Galaxy Information . . . . . . . . . . . . . . . 7

2 VLA Observing Information $\ldots \ldots \ldots \ldots$

$3 \quad$ VLA Map Information . . . . . . . . . . . . . . . . . 17

4 GBT Observing Information . . . . . . . . . . . . 23

5 GBT Map Information . . . . . . . . . . . . . . . 24

6 VLA Configuration Information . . . . . . . . . . . 111 


\section{LIST OF FIGURES}

FIGURE

PAGE

1 Haro 29 Left: FUV.; Right: V-band . . . . . . . . . . . . . . 25

2 Haro 29's integrated H I natural-weighted moment maps. Upper left: Integrated H I intensity map; contour levels are $1 \sigma \times(2,5,8,11,14,17,20,23$, 26, and 29) where $1 \sigma=8.47 \times 10^{19}$ atoms $\mathrm{cm}^{-2}$. Upper right: Velocity field; contour levels are $256.25 \mathrm{~km} \mathrm{~s}^{-1}$ to $306.25 \mathrm{~km} \mathrm{~s}^{-1}$ separated by 6.25 $\mathrm{km} \mathrm{s}^{-1}$. Bottom: Velocity dispersion field; contour levels are 5.18 to 20.72 $\mathrm{km} \mathrm{s}^{-1}$ separated by $2.59 \mathrm{~km} \mathrm{~s}^{-1}$. . . . . . . . . . . . . . 26

3 Haro 29's integrated H I robust-weighted moment maps. Upper left: Integrated H I intensity map; contour levels are $1 \sigma \times(2,4,6,8,10,12,14)$ where $1 \sigma=2.37 \times 10^{20}$ atoms $\mathrm{cm}^{-2}$. Upper right: Integrated $\mathrm{H}$ I intensity map colorscale and FUV contours. The single FUV source to the west of the other contours is a foreground star. Bottom: Integrated H I intensity map colorscale and V-band contours. The large sources to the west of the main V-band emission are foreground stars. . . . . . . . . . . . . . 27

4 Haro 29 Left: GBT H I column density; contour levels are $1 \sigma \times(5,10,15$, 20, 25, 30) where $1 \sigma=4.48 \times 10^{17}$ atoms $\mathrm{cm}^{-2}$. Right: The blanked GBT H I velocity field. . . . . . . . . . . . . . . . . . . . . . 29

5 Haro 29: The left column contains the P-V diagram and the right column contains the natural-weighted integrated $\mathrm{H}$ I intensity map with a red arrow indicating the location of the corresponding slice through the galaxy and pointing in the direction of positive offset. The slice is taken to go through

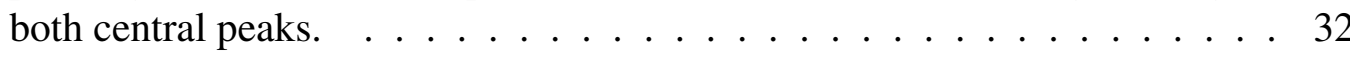

6 Haro 36 Left: FUV.; Right: V-band; the streak coming from the top-right of the image is due to a foreground star in the image. . . . . . . . . . 35

7 Haro 36's integrated H I natural-weighted moment maps. Upper left: Integrated H I intensity map; contour levels are $1 \sigma \times(2,4,6,8,10,12$, $14,16,18,20,22,24,26,28,30,32,34,36,38)$ where $1 \sigma=9.15 \times$ $10^{19}$ atoms $\mathrm{cm}^{-2}$. Upper right: Intensity weighted velocity field; contour levels are $445.5 \mathrm{~km} \mathrm{~s}^{-1}$ to $539 \mathrm{~km} \mathrm{~s}^{-1}$ separated by $5.5 \mathrm{~km} \mathrm{~s}^{-1}$. Bottom: Velocity dispersion field; contour levels are 11.28 to $25.38 \mathrm{~km} \mathrm{~s}^{-1}$ at intervals of $2.82 \mathrm{~km} \mathrm{~s}^{-1}$. 
8 Haro 36's integrated H I robust-weighted moment maps. Upper left: Integrated H I intensity map; contour levels are $1 \sigma \times(2,4,6,8,10,12,14,16$, 18) where $1 \sigma=2.79 \times 10^{20}$ atoms $\mathrm{cm}^{-2}$. Upper right: Integrated $\mathrm{H} \mathrm{I}$ intensity map colorscale and FUV contours. Bottom: Integrated H I intensity map colorscale and V-band contours. . . . . . . . . . . . . . . . . . . 37

9 Haro 36 Left: GBT H I column density; contour levels are $1 \sigma \times(4,5,10$, $15,20,25,30,35)$ where $1 \sigma=5.34 \times 10^{17}$ atoms cm ${ }^{-2}$. Right: GBT H I velocity field. . . . . . . . . . . . . . . . . 39

10 Haro 36: The left column contains the P-V diagrams and the right column contains the natural-weighted integrated H I map with a red arrow indicating the location of the corresponding slice through the galaxy and pointing in the direction of positive offset. The first row is a slice through both central peaks, the second row corresponds to the optical major axis. . . . .

11 Haro 36: The left column contains the P-V diagrams and the right column contains the natural-weighted integrated H I map with a red arrow indicating the location of the corresponding slice through the galaxy and pointing in the direction of positive offset. The first row is a slice through the northern extension and the northeast peak. The second row is a slice through the northern extension and the southwest peak. . . . . . . . . . . . . . 42

12 Haro 36: The top row contains the natural-weighted (left) and robustweighted (right) integrated H I maps with red lines indicating the approximate locations of the slices through the galaxy used in the P-V diagrams (a-d) located below. . . . . . . . . . . . . . . . . . . . . . 44

13 Haro 36: A labeled version of P-V diagram $\mathrm{b}$ from Figure 12. . . . . . . . 45

14 Haro 36: The V-band emission seen in Figure 6; the red circles indicate the location of features that may be a result of the tidal tail in Haro 36. . . . . . 48

15 Mrk 178 Left: FUV.; Right: H $\alpha$; Bottom: V-band. . . . . . . . . . . . 49

16 Mrk 178's natural-weighted moment maps. Upper left: Integrated H I intensity map; contour levels are $1 \sigma \times(2,4,6,8,10,12,14,16,18,20)$ where $1 \sigma=6.68 \times 10^{19}$ atoms $\mathrm{cm}^{-2}$. Upper right: Intensity weighted velocity field; contour levels are $235 \mathrm{~km} \mathrm{~s}^{-1}$ to $260 \mathrm{~km} \mathrm{~s}^{-1}$ separated by $2.5 \mathrm{~km} \mathrm{~s}^{-1}$. Bottom: Velocity dispersion field; contour levels are $9,11.5,14 \mathrm{~km} \mathrm{~s}^{-1}$. . . 50 
17 Mrk 178's robust-weighted moment maps. Upper left: Integrated H I intensity map; contour levels are $1 \sigma \times(2,4,6,8,10,12)$ where $1 \sigma=$ $1.95 \times 10^{20}$ atoms $\mathrm{cm}^{-2}$. Upper right: Integrated $\mathrm{H}$ I intensity map colorscale and FUV contours. Bottom left: Integrated H I intensity map colorscale and $\mathrm{H} \alpha$ contours. Bottom right: Integrated $\mathrm{H}$ I intensity map colorscale and V-band contours. . . . . . . . . . . . . . .

18 Mrk 178's GBT moment maps. Upper left: Integrated H I intensity map; contour levels are $1 \sigma \times(5,8,11,14,17,20,23,26,29,32,35,38,41$, $44,47)$ where $1 \sigma=2.89 \times 10^{17}$ atoms $\mathrm{cm}^{-2}$. Upper right: Intensity weighted velocity field Bottom: A comparison between Mrk 178's GBT data and natural weighted VLA data; the GBT data are the colorscale and grey contour of $1.5 \times 10^{18}$ atoms $\mathrm{cm}^{-2}$, and the VLA data are outlined by the red contour of $1.3 \times 10^{20}$ atoms $\mathrm{cm}^{-2}$. . . . . . . . . . 54

19 Mrk 178: The left column contains the P-V diagrams and the right column contains the natural-weighted integrated $\mathrm{H}$ I map with a red arrow indicating the location of the corresponding slice through the galaxy and pointing in the direction of positive offset. The first row is a slice through the hole in Mrk 178, the second row is a slice through the southern H I peak, and the third row is a slice through the northern H I peak. . . . . . . . . . . 56

20 VII Zw 403 Left: FUV.; Right: V-band. . . . . . . . . . . . . . . 61

21 VII Zw 403's natural-weighted moment maps. Upper left: Integrated H I intensity map; contour levels are $1 \sigma \times(2,6,10,14,18,22,26,30,34,38$, $42,46,50,54,58,62,66,70)$ where $1 \sigma=3.14 \times 10^{19}$ atoms $\mathrm{cm}^{-2}$. Upper right: Intensity weighted velocity field; contour levels are $90 \mathrm{~km} \mathrm{~s}^{-1}$ to 130 $\mathrm{km} \mathrm{s}^{-1}$ separated by $2.5 \mathrm{~km} \mathrm{~s}^{-1}$. Bottom: Velocity dispersion field; contour levels are $3 \mathrm{~km} \mathrm{~s}^{-1}$ to $15.5 \mathrm{~km} \mathrm{~s}^{-1}$ separated by $2.5 \mathrm{~km} \mathrm{~s}^{-1}$. . . . . . . . .

22 VII Zw 403's robust-weighted moment maps. Upper left: Integrated H I intensity map; contour levels are $1 \sigma \times(2,6,10,14,18,22,26)$ where $1 \sigma=1.59 \times 10^{20}$ atoms $\mathrm{cm}^{-2}$. Upper right: Integrated $\mathrm{H} \mathrm{I}$ intensity map colorscale and FUV contours. Bottom: Integrated H I intensity map colorscale and V-band contours. . . . . . . . . . . . . . . . 64

23 VII Zw 403 Left: GBT H I column density; contour levels are $1 \sigma \times(7,17$, $27,37,47,57,67,77,87)$ where $1 \sigma=1.59 \times 10^{17}$ atoms $\mathrm{cm}^{-2}$. Right: GBT H I velocity field. . . . . . . . . . . . . . . . . . 65

24 VII Zw 403's $25^{\prime \prime} \times 25^{\prime \prime}$ convolved map. . . . . . . . . . . . . . 66 
25 VII Zw 403's channel maps from the natural weighted VLA data cube where some overlap of VII Zw 403's and the Milky Way's velocity range occurs. . . . . . . . . . . . . . . . . . . . . . 67

26 The channel maps from the natural weighted VLA data cube from which the extra cloud emission south of VII Zw 403's main body comes. . . . . . 68

27 Left: The colorscale of VII Zw 403's VLA H I velocity field and contours from the VLA H I velocity dispersion map from Figure 21. Right: The colorscale of VII Zw 403's robust weighted VLA integrated H I intensity map overlaid with the velocity field contours from Figure $21 . \ldots . .$.

28 VII Zw 403: The left column contains the P-V diagrams and the right column contains the natural weighted integrated $\mathrm{H}$ I map with a red arrow indicating the location of the corresponding slice through the galaxy and pointing in the direction of positive offset. The slice goes through the velocity disturbance in VII Zw $403 . \ldots \ldots \ldots$. . . . . . . . . 70

29 VII Zw 403: Upper Left: Intensity weighted velocity field of VII Zw 403 without the emission from foreground gas cloud. The contours are -127.5 $\mathrm{km} \mathrm{s}^{-1}$ to $-85.0 \mathrm{~km} \mathrm{~s}^{-1}$ in intervals of $2.5 \mathrm{~km} \mathrm{~s}^{-1}$. Upper Right: The intensity weighted velocity field of the foreground gas cloud. The contours are $-125.0 \mathrm{~km} \mathrm{~s}^{-1}$ to $-92.5 \mathrm{~km} \mathrm{~s}^{-1}$ in intervals of $2.5 \mathrm{~km} \mathrm{~s}^{-1}$. Bottom: The contours from the velocity field in the upper left and colorscale of the velocity field in the upper right.

30 VII Zw 403: The left column contains the P-V diagram and the right column contains the robust-weighted integrated H I map with a red arrow indicating the location of the corresponding slice through the galaxy and pointing in the direction of positive offset. The slice goes through a potential hole (outlined in black). . . . . . . . . . . . . . . . . . 73

31 VII Zw 403: Left: The colorscale of the integrated H I intensity map of the disk without the foreground gas cloud with contours of the V-band data. Right: The colorscale of the integrated H I intensity map of the foreground gas cloud with contours of the integrated H I intensity map of the rest of the disk . . . . . . . . . . . . . . . . . . . 74

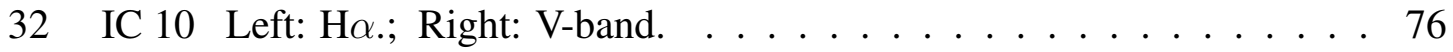

33 IC 10's natural-weighted moment maps. Upper left: Integrated H I intensity map blanked at a $2 \sigma$ level where $1 \sigma=14.7 \times 10^{19}$ atoms $\mathrm{cm}^{-2}$. Upper right: Intensity weighted velocity field. Bottom: Velocity dispersion field. . 77 
34 A colorscale map of IC 10's natural-weighted, main disk H I column density overlaid with stellar contours (note this map is on a different scale than those in Figure 33): Left: H $\alpha$. Right: V-band. . . . . . . . . . . . . . . . . 78

35 IC 10 Upper Left: GBT integrated H I intensity map; contour levels are $1 \sigma \times(8,20,60,100,140,180,220,260,300,340,380,420,460,500,540$, 580, 620) where $1 \sigma=4.76 \times 10^{17}$ atoms $\mathrm{cm}^{-2}$. Upper Right: GBT integrated $\mathrm{H}$ I intensity map from the upper left with the $2 \sigma$ contour of the VLA natural weighted column density map in red. Bottom Left: GBT H I velocity field. Contours are $-360 \mathrm{~km} \mathrm{~s}^{-1}$ to $-299.5 \mathrm{~km} \mathrm{~s}^{-1}$ separated by $5.5 \mathrm{~km} \mathrm{~s}^{-1}$. Bottom Right: The GBT velocity field in the bottom left with the $2 \sigma$ contour of the VLA natural weighted column density map in black. . 79

36 IC 10 Left: GBT integrated H I intensity map of the northern extension; the velocity range used for integration is -406.8 to $-373.0 \mathrm{~km} \mathrm{~s}^{-1}$. Contour levels are $1 \sigma \times(6,20,35,50,65,80,95,110,125,140,155,170,185,200)$ where $1 \sigma=1.1 \times 10^{17}$ atoms $\mathrm{cm}^{-2}$. Right: GBT H I velocity field of the northern extension map. Contours are -393 to $-382.5 \mathrm{~km} \mathrm{~s}^{-1}$ separated by $1.5 \mathrm{~km} \mathrm{~s}^{-1} \ldots \ldots$. . . . . . . . . . . . . . . . . 80

37 A labeled version of Figure 36 showing the location of the features in the case that the northern extension is a bridge and companion galaxy. . . . . 83

38 A labeled version of Figure 36 showing the location of the features in the case that the northern extension is a tidal tail and the eastern $\mathrm{H}$ I peak is a

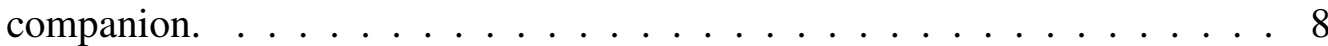

39 Velocity channels from IC 10's GBT data cube that clearly show the eastern peak. In the velocity range shown, these channels are every fifth channel in the data cube. . . . . . . . . . . . . . . . . . . 86

40 IC 10: The left column contains the P-V diagram and the right column contains the GBT integrated H I map from Figure 36 with a red arrow indicating the location of the corresponding slice through the galaxy and pointing in the direction of positive offset. This slice goes through both central peaks seen in the GBT data. . . . . . . . . . . . . . . 87

41 Velocity field of IC 10's GBT data without the southern plume or most of the western peak. . . . . . . . . . . . . . . . . 88

42 A schematic of a potential viewing angle of IC 10 . . . . . . . . . . . 89

43 Velocity channel maps for IC 10's southern plume at intervals of 12 channels. 91

44 NGC 3738 Left: FUV.; Right: V-band. . . . . . . . . . . . . . . . . 93 
45 NGC 3738's natural-weighted moment maps. Upper left: Integrated H I intensity map; contour levels are $1 \sigma \times(2,8,14,20,26,32)$ where $1 \sigma=$ $1.01 \times 10^{20}$ atoms $\mathrm{cm}^{-2}$. Upper right: Intensity weighted velocity field; contour levels are $165 \mathrm{~km} \mathrm{~s}^{-1}$ to $275 \mathrm{~km} \mathrm{~s}^{-1}$ separated by $10 \mathrm{~km} \mathrm{~s}^{-1}$. Bottom: Velocity dispersion field; contour levels are $5 \mathrm{~km} \mathrm{~s}^{-1}$ to $35 \mathrm{~km} \mathrm{~s}^{-1}$ separated by $5 \mathrm{~km} \mathrm{~s}^{-1}$. . . . . . . . . . . . . . . . . . . . 94

46 NGC 3738's natural-weighted moment maps. Upper left: Integrated H I intensity map; contour levels are $1 \sigma \times(2,6,10,14,18)$ where $1 \sigma=3.27 \times$ $10^{20}$ atoms $\mathrm{cm}^{-2}$. Upper right: Integrated $\mathrm{H}$ I intensity map colorscale and FUV contours. Bottom: Integrated H I intensity map colorscale and V-band contours.

47 NGC 3738's GBT moment maps. Left: Integrated H I intensity map; contour levels are $1 \sigma \times(7,17,27,37,47,57,67,77,87,97,107,117,127)$ where $1 \sigma=2.731 \times 10^{17}$ atoms $\mathrm{cm}^{-2}$. Right: Intensity weighted velocity

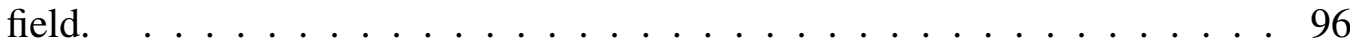

48 NGC 3738's $25^{\prime \prime} \times 25^{\prime \prime}$ convolved map. . . . . . . . . . . . . . . . 97

49 NGC 3738: The left column contains the P-V diagram and the right column contains the natural-weighted integrated H I map with a red arrow indicating the location of the corresponding slice through the galaxy and pointing in the direction of positive offset. This slice shows a counter rotating gas cloud found in the line of sight of NGC 3738's body. The location of the counter-rotating gas is indicated on the red arrow by a white line and circled in the $\mathrm{P}-\mathrm{V}$ diagram. . . . . . . . . . . . . . . . . . . 98

50 The Earth is represented by the largest circle. Two antennae, the blue and purple circles, have different baselines (the white line) as the earth rotates. . 111

51 Sine wave sampling rate example, where the pink circles represent Nyquist sampling and the blue circles represent undersampling. The simplest fits to these sampling rates are shown in the corresponding colors of the circles and the black sine wave represents the original sine wave. . . . . . . . 115

52 The emission from a source that was reflected into NGC 3738's GBT target frequency range. . . . . . . . . . . . . . . . . 117

53 Top: An example of a bad baseline fit using channels (0-250; 674-720; 1070-1380; 1820-2062). Bottom: An example of a good baseline fit using channels $(0-250 ; 674-900 ; 1820-2062)$. . . . . . . . . . . . . 118

54 The averaged spectra of NGC 3738 from the GBT data. . . . . . . . . 120 
55 The averaged spectra of a second galaxy that is reflected into the target frequency range of NGC $3738 \ldots \ldots \ldots 121$ 


\section{LIST OF SYMBOLS}

D: Distance of the galaxy

$\mathrm{E}_{0}$ : The initial energy needed to create an $\mathrm{H}$ I hole in units of $10^{50} \mathrm{ergs}$

$\mathrm{E}_{51}$ : The energy emitted by a supernova explosion in units of $10^{51} \mathrm{ergs}$

$\mathrm{M}\left(\mathrm{M}_{\odot}\right): \mathrm{H}$ I mass in units of solar masses

$\mathrm{M}_{V}: \mathrm{V}$-band magnitude

$\mathrm{N}$ : Number of velocity channels with signal

number $_{\text {boxcar }}$ : Number of channels averaged in a boxcar function

number $_{n c}$ : Number of new channels after boxcar smoothing

number $_{o c}$ : Number of original channels before boxcar smoothing

$\mathrm{N}_{*}$ : Number of stars needed to create an $\mathrm{H}$ I hole

$\mathrm{n}_{0}$ : The initial volumetric density of the gas

off: Telescope readout when the telescope is off frequency

on: Telescope readout when the telescope is off frequency

R: Radius of an $\mathrm{H}$ I hole

$\mathrm{R}_{D}$ : The V-band disk scale length

$\mathrm{S}_{i}$ : Flux per a velocity channel

$\mathrm{SFR}_{D}$ : The star formation rate, measured from FUV data (or $\mathrm{H} \alpha$ data), normalized to an area of $\pi \mathrm{R}_{D}^{2}$.

$\mathrm{t}_{7}$ : timescale for an $\mathrm{H}$ I hole to be created in units of $10^{7}$ years

$\mathrm{T}_{a}$ : Antenna temperature 
$\mathrm{T}_{i}$ : Temperature per a velocity channel

$\mathrm{T}_{\text {ISPEC }}$ : The output temperature from ISPEC

$\mathrm{T}_{\text {sys }}:$ System temperature

$\mathrm{v}_{s h}$ : The velocity of an H I hole's expansion

$\Delta \mathrm{V}$ : The velocity channel width

$\Delta \mathrm{x}$ : The $\mathrm{x}$-dimension of the GBT pixel size

$\Delta y$ : The y-dimension of the GBT pixel size

$\Delta \alpha:$ FWHM of the major axis of the beam

$\Delta \delta$ : FWHM of the minor axis of the beam

$\sigma:$ Integrated noise level

$\sigma_{\text {chan }}$ : Average noise per a velocity channel 


\section{LIST OF ABBREVIATIONS AND ACRONYMS}

AIPS: Astronomical Image Processing System

BCD: Blue Compact Dwarf

dIrr: Dwarf Irregular

DSS: Digitized Sky Survey

EVLA: Extended VLA

FIGGS: Faint Irregular Galaxies GMRT Survey

FUV: Far-Ultraviolet

FWHM: Full-Width Half-Maximum

GALEX: Galaxy Evolution Explorer

GBT: Green Bank Telescope

GMRT: Giant Metrewave Radio Telescope

H I: Atomic Hydrogen

IGM: Intergalactic Medium

ISM: Interstellar Medium

LITTLE THINGS: Local Irregulars That Trace Luminosity Extremes, The H I Nearby Galaxy Survey

M-S: Multi-Scale

NED: NASA/IPAC Extragalactic Database

PA: Position angle

PNe: Planetary nebulae 
P-V: Position-Velocity

RA: Right Ascension

RFI: Radio Frequency Interference

RMS: Root Mean Square

VLA: Very Large Array

WR: Wolf-Rayet

WSRT: Westerbork Synthesis Radio Telescope 


\section{Introduction}

Dwarf galaxies are low mass, low shear galaxies that have solid body rotation. There are several types of dwarf galaxies including gas rich dwarf irregular (dIrr), and gas poor dwarf elliptical galaxies. Blue compact dwarf (BCD) galaxies are undergoing a burst of star formation that gives them bluer colors and a more compact appearance on photographic plates than other dwarf galaxies. Yet, dwarf galaxies tend to be inefficient star formers (Leroy et al. 2008), so what triggers the burst of star formation seen in BCDs but not other dwarf galaxies? In general, star formation occurs when the gas in the galaxy (mostly atomic hydrogen; H I) is disturbed enough to create regions of high density in the gas. These high density regions of gas become cold molecular clouds that can then collapse under gravity to form stars. Gas rich, low metallicity, low shear dwarf galaxies, such as dIrr and BCD galaxies, are thought to rely heavily upon local processes to regulate their star formation (e.g., turbulence and previous star formation injecting energy into the interstellar medium). Yet, how much each process contributes to regulating star formation in dwarf galaxies is currently unknown. What then goes on to trigger the burst of star formation in BCDs is also not well understood.

It has been suggested that the high star formation rates in BCDs are a result of interactions with other galaxies, dwarf-dwarf galaxy mergers, or consumption of intergalactic gas (Taylor 1997; Wilcots \& Miller 1998; Noeske et al. 2001; Pustilnik et al. 2001; Martínez-Delgado et al. 2012). Simulations of these processes have shown that they will produce high rates of star formation and dense regions of star formation such as those observed in BCDs (Noguchi 1988a,b; Bekki 2008). However, there are BCDs that are thought to be isolated (Taylor 1997; Nicholls et al. 2011; Simpson et al. 2011; Ashley et al. 2013) making an encounter with another galaxy or gas cloud unlikely. 
Finding the trigger mechanism for high star formation rates in BCDs could allow us to better understand galaxy evolution. Past research has had little success in placing BCDs on evolutionary tracks that allow them to evolve from or into other types of dwarf galaxies (Papaderos et al. 1996; van Zee 2001; Tajiri \& Kamaya 2002; Gil de Paz \& Madore 2005). Understanding what has triggered the starburst in BCDs should help us model how BCDs evolve and their relationship to other types of dwarf galaxies. Also, in the bottom-up theory of galaxy evolution, massive galaxies are built up by cold accretion and possibly dwarf mergers (for a review of current galaxy formation theory see Benson 2010). Therefore, if BCDs are the result of mergers or accretion, then they may be useful analogs for studying early galaxy formation.

Distinguishing properties of mergers and interactions include bridges and tidal tails. Toomre \& Toomre (1972) explained these features by considering a disk galaxy and a point source perturber. As the perturber comes within close proximity to the disk galaxy, its gravitational force will begin to pull material from the outskirts of the disk. The extracted material can create a temporary bridge between the disk and the perturber (see Figure 4 of Toomre \& Toomre 1972). As the perturber gets further from the disk galaxy, the bridge between the two galaxies will dissipate. Because tidal forces are two-sided (e.g., the tides of the ocean), the side of the disk opposite of the perturber's location will also have material pulled out from it, creating a feature called a tidal tail. Bridges and tidal tails are clear signatures of an interaction or merger and can appear in both of the galaxies involved. The properties of the tidal features such as length, lifetime, and angle to the disk depend greatly on the properties of the two galaxies interacting and the properties of the interaction. For example: relative mass of the galaxies, the angle of incidence, and direction of passage vs. the direction of the galaxies' rotation (retrograde/prograde). If the galaxies interacting have disks that are counter-rotating with respect to one another, 
and they accrete gas from one another or go on to merge, then they will also have counter-rotating gas in their system.

Understanding the effects of star formation on the gas is also important for understanding the evolution of galaxies, especially in galaxies with high star formation rates like BCDs. For example, massive stars can give off a significant amount of radiation called a stellar wind that can ionize and exert an outward force on the surrounding interstellar medium (ISM). Many of these massive stars in one area, combined with supernova explosions ${ }^{2}$ can create large holes in the ISM surrounding the stellar population. As the ISM is cleared out of the area surrounding the stellar population, a snow plow effect can occur, where the gas that used to be in the hole is being pushed outwards into the surrounding gas, building a high density shell around the hole. To an observer, the shell will have a circular appearance because the observer is seeing a bubble shape in projection. Therefore, the observer will be looking through the most gas at the edges of the projected circle and these edges will have a higher column density in the $\mathrm{H}$ I maps. The shell that is left surrounding the hole may then be dense enough to form its own stars after it has had enough time to cool. Holes can even break through the disk of the galaxy. When a hole breaks through the disk material can flow out of the disk of the galaxy creating a galactic chimney or fountain and may even reach escape velocity, becoming part of the intergalactic medium (IGM). The ejection of material from dwarf galaxy disks has been suggested as a means of releasing metals into the IGM, thereby keeping the metallicity of dwarf galaxies low despite having had previous generations of stars which should pollute the ISM with metals (Marlowe et al. 1995; Mac Low \& Ferrara 1999).

\footnotetext{
${ }^{2}$ Supernova explosions occur two ways: when a white dwarf in a binary system accretes too much mass from its companion and has a runaway fusion event creating an explosion, or when massive stars run out of fuel for fusion. In the latter case, the massive star will no longer be able to produce enough outward force to prevent the gravitational collapse of the star. When this collapse occurs, the outer layers of the star bounce off of the dense inner core creating an explosion.
} 
In this dissertation I will be studying six apparently isolated BCDs from the LITTLE THINGS ${ }^{3}$ sample to try and understand what has triggered their bursts of star formation. I will analyze the gas kinematics and morphology of their high angular and velocity resolution Very Large Array ${ }^{4}$ (VLA) H I data to look for signatures of interactions, advanced mergers, impacting gas clouds, and other external disturbances. Signatures of these could include a tidal tail resulting from a past interaction, counter-rotating gas clouds being accreted onto the galaxy, gas kinematics that depart significantly from solid body rotation (dwarf galaxies tend to be solid body rotators), or gas morphology that appears disturbed. I then use low resolution, high sensitivity Robert C. Byrd Green Bank Telescope $^{4}$ (GBT) H I data to search for nearby companions, intergalactic gas clouds, or previously unseen tenuous extensions of these BCDs. ${ }^{5}$

\footnotetext{
${ }^{3}$ Local Irregulars That Trace Luminosity Extremes, The H I Nearby Galaxy Survey; https://science.nrao.edu/science/surveys/littlethings

${ }^{4}$ The National Radio Astronomy Observatory is a facility of the National Science Foundation operated under cooperative agreement by Associated Universities, Inc.

${ }^{5}$ Two papers have already been published that include some of the results in this dissertation: Ashley et al. (2013) and Nidever et al. (2013).
} 


\section{Sample}

The sample in this dissertation is a subsample from LITTLE THINGS. LITTLE THINGS is a VLA H I dwarf galaxy survey that includes 41 dIrr and BCD galaxies. The purpose of the survey is to understand star formation in dwarf galaxies. Each galaxy in the LITTLE THINGS sample is thought to be relatively isolated from other galaxies, as they were chosen to be galaxies that are not obviously interacting with other galaxies. The subsample contains six BCDs: Haro 29, Haro 36, Mrk 178, VII Zw 403, IC 10, and NGC 3738. Basic information for each BCD is listed in Table 1.

\subsection{Haro 29}

Haro 29 (=Mrk 209=I Zw 36=UGCA 281) is located roughly in the Canes Venetici I group of galaxies (Kaisin et al. 2008); a loosely gravitationally bound group of galaxies. The closest known neighbor to Haro 29 within $\pm 150 \mathrm{~km} \mathrm{~s}^{-1}$ is NGC 4144 (Hunter \& Elmegreen 2004) at a distance of $410 \mathrm{kpc}$ and a velocity difference of $16 \mathrm{~km} \mathrm{~s}^{-1}$. Companions that are currently interacting are not likely to be more than $\sim 100 \mathrm{kpc}$ away from each other and are likely to be close in velocity (Taylor et al. 1995; Pustilnik et al. 2001; Thilker et al. 2004; Westmeier et al. 2008; Chynoweth et al. 2011). Haro 29's H I data has been observed twice before with the Westerbork Synthesis Radio Telescope (WSRT; Viallefond \& Thuan 1983; Stil \& Israel 2002). The integrated H I intensity maps from Viallefond \& Thuan (1983) have an angular resolution of 37'.2 and show a clumpy core-halo structure with an organized inner velocity field. The integrated H I intensity maps from Stil \& Israel (2002) have a higher angular resolution of $13^{\prime \prime}$ and show two H I peaks in the central region of the galaxy. Neither of the studies made conclusions about what may have triggered Haro 29's starburst. 
Haro 29's stellar population has also been extensively studied by Schulte-Ladbeck et al. (2001). In that study, Hubble Space Telescope data were used to obtain a distance for Haro 29 and a star formation history. Schulte-Ladbeck et al. (2001) found that over the past Gyr Haro 29 has likely had an increase in its star formation rate by 13 times to $2.5 \times 10^{-2} M_{\odot} \mathrm{yr}^{-1}$ when compared to its modeled star formation rate prior to $1 \mathrm{Gyr}$. Haro 29's youngest stellar population has an age of only a few Myr, which is evident by the Wolf-Rayet (WR) stars in Haro 29 (Izotov et al. 1997). Wolf-Rayet stars are massive stars $\left(\geq 20 \mathrm{M}_{\odot}\right)$ that are very luminous and have strong stellar winds. Because of their mass, these stars quickly consume their fuel for fusion and leave the WR stage of their lives within a few Myr of having formed; they are therefore signatures of star formation having occurred in the past few Myr.

\subsection{Haro 36}

At $9.3 \mathrm{Mpc}$, Haro 36 (=UGC 7950) is the most distant galaxy in my sample. The closest known companion to Haro 36 is NGC 4707 at a distance of $\sim 530 \mathrm{kpc}$ and a velocity difference of $34 \mathrm{~km} \mathrm{~s}^{-1}$ (Hunter \& Elmegreen 2004). Haro 36 has been included in several large surveys (e.g., Kennicutt et al. 2008; Dale et al. 2009; Lee et al. 2011), however, very few studies have studied it as an individual galaxy. Simpson \& Gottesman (2000) included Haro 36 in an H I study that used VLA data with an angular resolution of 26 ".7 and velocity resolution of $12.5 \mathrm{~km} \mathrm{~s}^{-1}$. They concluded that Haro 36 has an $\mathrm{H}$ I disk with an elongated, unresolved central peak which Simpson \& Gottesman (2000) suggested may be two separate, unresolved peaks. Hunter \& Elmegreen (2006) also briefly mention Haro 36 in their optical dwarf galaxy survey, noting that it has an elongated blue stellar component along the stellar major axis. 


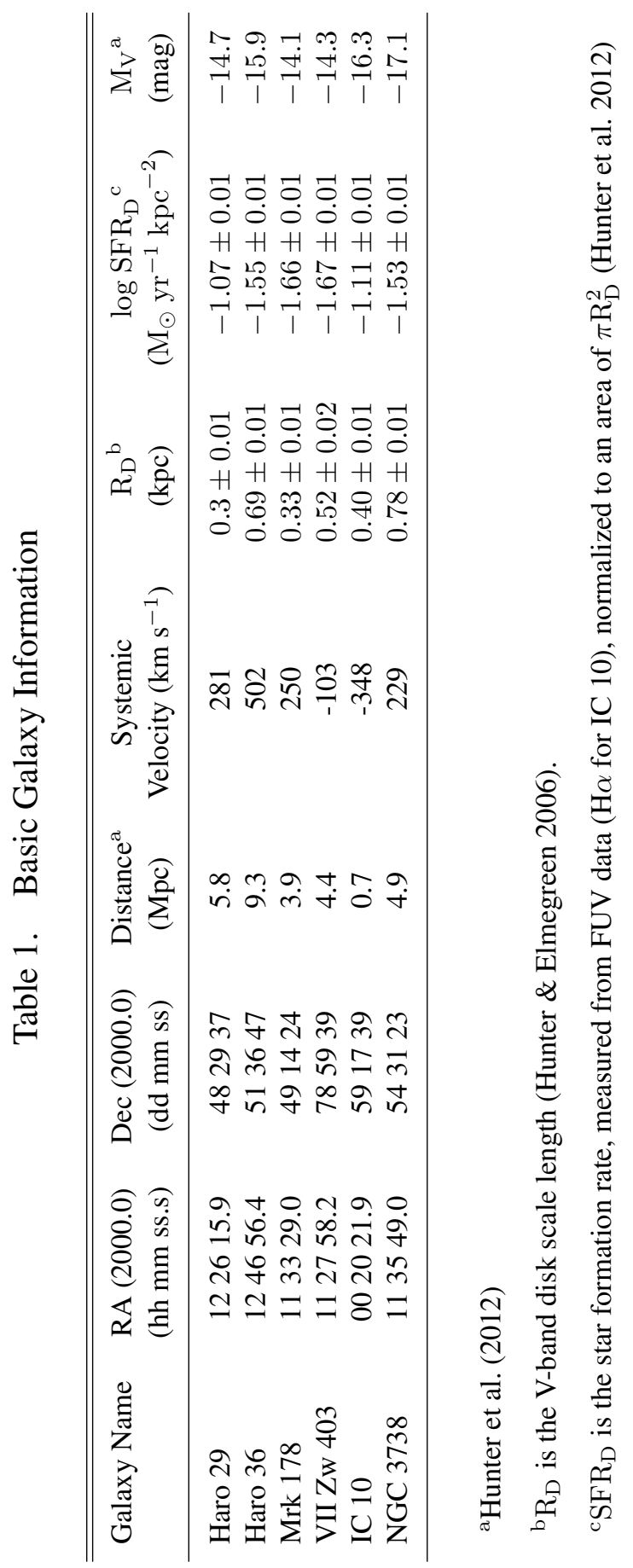




\section{3. $\quad$ Mrk 178}

Mrk 178 (=UGC 6541) is a galaxy that has a confusing classification record in journal papers; it has been classified as a merger (Pustilnik et al. 2001) and two separate articles have suggested that Mrk 178 has a nearby companion (a different companion in each paper) at a velocity close to its own velocity (Peterson 1979; Thuan \& Martin 1981). Upon further investigation, none of these claims can be verified. Pustilnik et al. (2001) appear to cite Mazzarella et al. (1991) to support the idea that Mrk 178 is a merger, however, Mazzarella et al. (1991) do not claim that it is a merger, merely a dwarf irregular. It is possible that the confusion over Mrk 178's classification in Pustilnik et al. (2001) came from Mazzarella et al. (1991) searching for mergers in their paper and having a very large sample size. Thuan \& Martin (1981) suggest that UGC 6538 is a companion to Mrk 178, however, the velocity difference of these two galaxies is almost $2800 \mathrm{~km} \mathrm{~s}^{-1}$ (velocities taken from $\mathrm{NED}^{6}$ ), making UGC 6538 a very unlikely companion galaxy and more likely a background galaxy. Peterson (1979) suggest that Mrk 178 is a close pair with UGC 6549 , however, the velocity difference between these two galaxies is more than $9000 \mathrm{~km}$ $\mathrm{s}^{-1}$ (velocities taken from NED), making it a very unlikely candidate for a companion to Mrk 178 and more likely a spatially coincident background galaxy. The classification of Mrk 178 in Peterson (1979) has led NED to label Mrk 178 as a galaxy in a pair. Hunter \& Elmegreen (2004) find, from a NED search, that the closest galaxy to Mrk 178 within $\pm 150 \mathrm{~km} \mathrm{~s}^{-1}$ is NGC 3741 at a distance of $410 \mathrm{kpc}$ and a velocity difference of $20 \mathrm{~km}$ $\mathrm{s}^{-1}$. Mrk 178 is located roughly in the Canes Venetici I group of galaxies (Karachentsev et al. 2003).

The stellar and gas components of Mrk 178 have been previously studied (Stil \& Israel 2002; Roychowdhury et al. 2009; Lelli 2013; Kehrig et al. 2013). Stil \& Israel

\footnotetext{
${ }^{6}$ NASA/IPAC Extragalactic Database (NED) http://ned.ipac.caltech.edu/
} 
(2002) studied Mrk 178's H I data using WSRT. Their integrated H I intensity map, at a resolution of $13^{\prime \prime}$, shows a broken ring-like structure. Mrk 178 is also part of a survey that uses the Giant Metrewave Radio Telescope (GMRT) called the Faint Irregular Galaxies GMRT Survey (FIGGS). Roychowdhury et al. (2009) present Mrk 178's FIGGS data, but do not discuss the morphology or kinematics of Mrk 178 as an individual galaxy. Their integrated H I intensity maps, at resolutions of $22.68^{\prime \prime}$ and $11.71^{\prime \prime}$, show that Mrk 178 has a highly irregular shape to its gaseous disk.

Mrk 178's stellar population is well known for its young WR features (SchulteLadbeck et al. 2000; Guseva et al. 2000; Brinchmann et al. 2008; Stevens \& Strickland 1998; Kehrig et al. 2013). A detailed study of Mrk 178's WR population by Kehrig et al. (2013) revealed a large number of WR stars in its brightest stellar component. Mrk 178's stellar population was studied in detail by Schulte-Ladbeck et al. (2000). Their results indicate that Mrk 178 had a higher star formation rate $\sim 0.5$ Gyr ago when compared to its current star formation rate. Their results also indicate that Mrk 178 has an old underlying stellar population.

\subsection{Zw 403}

VII Zw 403 (=UGC 6456) is a well known isolated BCD without obvious signatures of tidal interaction (Schulte-Ladbeck \& Hopp 1998; Noeske et al. 2001; Pustilnik et al. 2001; Simpson et al. 2011). It sits just beyond the M81 group and is accelerating back towards the M81 group (Karachentsev et al. 2002). Hunter \& Elmegreen (2004) found that the closest galaxy to VII Zw 403 within $\pm 150 \mathrm{~km} \mathrm{~s}^{-1}$ is KDG 073 at a distance of 900 kpc and a velocity difference of $32 \mathrm{~km} \mathrm{~s}^{-1}$. VII Zw 403's $\mathrm{H}$ I has been studied using the GBT, Nançay, the GMRT, and the VLA (Tully et al. 1981; Paturel et al. 2003; Begum et

al. 2008; Roychowdhury et al. 2009; Thuan et al. 2004; Simpson et al. 2011). The H I data 
reveal a galaxy that has a disturbed velocity field and irregular morphology. The VLA data presented in Simpson et al. (2011) reveal a break in the major axis of the isovelocity contours and a possible H I hole. Simpson et al. (2011) conclude that VII Zw 403 may have experienced an H I accretion event in its past, which is now difficult to detect.

Schulte-Ladbeck et al. (2001) modeled the star formation history of VII Zw 403, concluding that its star formation has been continuous and not episodic, with an increased star formation rate over the past Gyr. Lynds et al. (1998) also modeled VII Zw 403's star formation history and showed a starburst occurring in the 600-800 Myr interval of the VII Zw 403's past, followed by a lower star formation rate. X-ray emission has also been detected in VII Zw 403 using the satellite ROSAT and its instrument PSPC (Papaderos et al. 1994). There was apparent extended X-ray emission in the form of three diffuse arms, which all emanated from a central source. A point source located at the central X-ray source was later confirmed by two studies (Lira et al. 2000; Ott et al. 2005), but the diffuse emission seen in Papaderos et al. (1994) was not detected by Chandra or ROSAT's HRI instrument.

\subsection{IC 10}

IC 10 (=UGC 192) is the closest galaxy to the Milky Way in my sample at a distance of $0.7 \mathrm{Mpc}$, and with currently over 400 published journal papers (listed in NED), it is also the most well-studied galaxy of my sample. Hunter \& Elmegreen (2004) indicate that its closest companion within $\pm 150 \mathrm{~km} \mathrm{~s}^{-1}$ is $\mathrm{M} 31$ at a distance of $250 \mathrm{kpc}$ and velocity difference of $48 \mathrm{~km} \mathrm{~s}^{-1}$. IC 10 is the only known starburst galaxy in the Local Group. It is a distant M31 satellite (Ibata et al. 2013; Tully 2013) and is close enough to have proper motion measurements from masers (Brunthaler et al. 2007). Its close proximity makes it an excellent subject to study, however, its observational proximity to the Galactic plane 
make it difficult to determine extinction. IC 10's extinction has led to some dispute over IC 10's classifaction since the colors of the galaxy are dependent upon the extinction of the galaxy. Richer et al. (2001) completed a detailed study of IC 10's extinction and concluded that IC 10 should be classified as a BCD. Therefore, for the purpose of not leaving out any possible BCDs in my sample, it has been included in the current sample.

The H I of IC 10 has been well studied (Huchtmeier 1979; Shostak \& Skillman 1989; Wilcots \& Miller 1998; Manthey \& Oosterloo 2008; Nidever et al. 2013), revealing an extended $\mathrm{H}$ I pool in the lower resolution high sensitivity data. The higher resolution, lower sensitivity data reveals a southern plume, three spurs around the galaxy, and many holes/shells. The H I data also reveal disturbed velocity fields in IC 10; the outer regions of the H I are counter-rotating with respect to the inner gas (Shostak \& Skillman 1989; Wilcots \& Miller 1998). Shostak \& Skillman (1989) and Wilcots \& Miller (1998) suggest that the counter-rotating gas and apparent isolation of IC 10 point to it being a galaxy that is an advanced merger or a galaxy that is still in formation and is still collecting the primordial gas surrounding it. Yin et al. (2010) modeled the chemical evolution of IC 10. They concluded that slow gas accretion on the timescale of $8 \mathrm{Gyr}$ is consistent with their models.

IC 10 also has a well studied stellar component. Massey \& Armandroff (1995) and Jarrett et al. (2003) measured a stellar disk diameter of 6-7', but Sanna et al. (2010) show that the disk diameter may be $20^{\prime}-46^{\prime}$, possibly larger. Massey \& Armandroff (1995) also detect an unusually high number of WR stars in IC 10; its disk contains at least $15 \mathrm{WR}$ stars while $1 \mathrm{WR}$ star is typical for a star-forming dwarf galaxy. The number of WR stars in IC 10 indicates that IC 10 is forming massive stars at the rate of a star-forming region in a spiral galaxy, such as M33, throughout its disk. Gonçlaves et al. (2012) used the Subaru telescope to study the kinematics of 35 planetary nebulae in IC 10 that traced the stellar motions in the outer H I. They concluded that the planetary nebulae generally follow the 
same kinematic trends as the gas as measured by VLA, including the kinematics of some of the outer gas.

\subsection{NGC 3738}

NGC 3738 (=UGC 6565) is another galaxy that has not received a significant amount of individual attention. NGC 3738 is not always classified as a BCD, however, Vaduvescu et al. (2006) suggest that the light profile properties of NGC 3738 are those of a BCD and Hunter \& Elmegreen (2006) also support this idea, with their light profile of NGC 3738 being most similar to the other eight BCDs in their sample. Therefore, it has been included in my BCD sample. NGC 3738 has been included in several other large H I surveys (Stil \& Israel 2002; Paturel et al. 2003), however, it is not discussed in detail. The map from Stil \& Israel (2002) reveals a very clumpy and irregular H I morphology at a resolution of 13.5". Hunter \& Elmegreen (2004) find that the closest companion to NGC 3738 within \pm $150 \mathrm{~km} \mathrm{~s}^{-1}$ is NGC 4068, at a distance of $490 \mathrm{kpc}$ and a velocity difference of $19 \mathrm{~km} \mathrm{~s}^{-1}$. NGC 3738 is located roughly in the Canes Venetici I group of galaxies (Karachentsev et al. 2003). 


\section{Observations and Data Reduction}

\subsection{The Very Large Array Telescope}

The LITTLE THINGS H I data were obtained using the VLA. The VLA is a radio interferometry telescope composed of up to twenty-seven dishes, each with a diameter of twenty-five meters. Together the 27 dishes simulate a large radio telescope (for more information on radio telescopes, radio interferometry, and the VLA as an interferometer see Appendices 1, 2, and 2.1). The LITTLE THINGS survey was granted $367 \mathrm{hr}$ at the VLA to obtain new data and also made use of archival VLA H I data when possible. All LITTLE THINGS galaxies have B, C, and D configuration data of high velocity $(\leq 2.6 \mathrm{~km}$ $\left.\mathrm{s}^{-1}\right)$ and angular $\left(\sim 6^{\prime \prime}\right)$ resolution. Basic information on the observational parameters for the BCD sample are given in Table 2.

The LITTLE THINGS data were taken during the upgrade of the VLA to the Extended VLA (EVLA). The upgrade improved observing capabilities by increasing the frequency coverage, increasing the sensitivity, and allowing for finer velocity resolution (for details see Perley et al. 2011). In order to keep the VLA in operation during the upgrade, which took several years, antennas were progressively upgraded, leaving some VLA antennas and some EVLA antennas during observations. The differences between the VLA and EVLA correlators caused unique problems discussed in detail in Hunter et al. (2012). One of the problems encountered was the inability to Doppler track; Doppler tracking corrects for the Doppler shift in a spectrum associated with the rotation of the earth. To make sure the data taken without Doppler tracking were still useful, checks were made during observations to make sure that the spectrum of each galaxy did not drift outside the observed frequency range (a.k.a. bandwidth).

A second problem that was encountered was deemed the "aliasing problem." The aliasing problem was caused by data from EVLA antennas being fed into the 
VLA correlator which then aliased power from outside the bandpass into the data over the beginning of the observed frequency range. The aliased power resulted in a lower signal-to-noise ratio in the data. For more details on the aliasing problem, see https://science.nrao.edu/facilities/vla/obsolete/aliasing. The aliasing problem significantly affected the EVLA-EVLA baselines. As a result all EVLA-EVLA baselines were removed from the data and more time was added to observations to compensate for loss of data, retaining the signal-to-noise.

The H I data were calibrated and mapped in Astronomical Image Processing System ${ }^{7}$ (AIPS) using the LITTLE THINGS calibration and mapping recipes discussed in Hunter et al. (2012). The mapping was done using the Multi-Scale (M-S) CLEAN in AIPS, a nonstandard CLEAN algorithm that is useful for recovering tenuous emission. Standard CLEAN recovers flux by looking for point sources with the highest flux, "cleaning" that point source by placing the components with the highest flux into an image file, then re-imaging and looking for the next point source of highest flux. CLEAN will continue to do this until it reaches a user-set limit of flux or runs out of components to clean ${ }^{8}$. In the nonstandard M-S CLEAN the data are first convolved ${ }^{9}$ to user-input synthesized beam sizes. For the LITTLE THINGS data these beam sizes are 0 (or no convolution), 15, 45, and 135 arcsec (for more information about beams, see Appendix 3). The CLEAN algorithm then searches for the region of highest flux amongst all of the convolved maps and then recovers clean components from the map with the region of highest flux. Larger beam sizes imply higher sensitivity and lower resolution, whereas smaller beams imply lower sensitivity

\footnotetext{
${ }^{7}$ AIPS is a program that consists of many subprograms that are designed for calibrating and mapping radio data. It is often used to calibrate and map VLA data. Documentation on AIPS can be found at http://www.aips.nrao.edu/index.shtml.

${ }^{8}$ For more information about standard CLEAN see Högbom (1974).

${ }^{9}$ Convolving here refers to the process of smoothing data spatially to a lower resolution and higher sensitivity with a gaussian function, a.k.a. a convolved beam.
} 
and higher resolution. Since M-S CLEAN uses multiple synthesized beams to retrieve clean components for the final maps, it is able to recover the high resolution structure like the standard CLEAN algorithm does, but with the added benefit of recovering clean components of higher sensitivity from the larger synthesized beams. For more information about the benefits of M-S CLEAN see Cornwell (2008).

In AIPS, natural weighted and robust weighted cubes ${ }^{10}$ of the $\mathrm{H}$ I data were made using IMAGR. The natural weighting scheme has a larger synthesized beam than the robust maps and thus have a higher sensitivity and lower resolution than the robust maps (Robust value $=0.5$; for details on natural vs. robust weighting, see Appendix 4). The cubes are then integrated along the velocity axis to make a set of 2D maps including an integrated $\mathrm{H} \mathrm{I}$ intensity map proportional to the column density of the $\mathrm{H} \mathrm{I}$, an intensity weighted $\mathrm{H} \mathrm{I}$ velocity field, and an H I velocity dispersion map. For basic information on the individual VLA maps, see Table 3.

\footnotetext{
${ }^{10} \mathrm{~A}$ cube here refers to a 3D map, where two of the dimensions are spatial and the third, discrete dimension is velocity.
} 
Table 2. VLA Observing Information

\begin{tabular}{lcccc}
\hline \hline Galaxy Name & Configuration & Date Observed & Project ID & Time on Source (hours) \\
\hline \multirow{5}{*}{ Haro 29 } & B & 08 Jan 28, 08 Jan 30 & AH927 & 9.2 \\
& CnB & 08 Feb 7 & AH927 & 1.7 \\
& C & 08 Mar 23, 08 Apr 19 & AH927 & 5.9 \\
\hline \multirow{3}{*}{ Haro 36 } & D & 08 Jul 6, 08 Jul 29 & AH927 & 1.7 \\
& B & 08 Jan 15, 08 Jan 21, 08 Jan 27 & AH927 & 10.3 \\
& C & 08 Mar 23, 08 Apr 15 & AH927 & 5.7 \\
Mrk 178 & D & 08 Jul 8, 08 Jul 24, 08 Jul 25 & AH927 & 2.2 \\
& B & 08 Jan 15, 08 Jan 21, 08 Jan 27 & AH927 & 10.4 \\
& C & 08 Mar 23, 08 Apr 15 & AH927 & 5.75 \\
VII Zw 403 & D & 08 Jul 8, 08 Jul 24, 08 Jul 25 & AH927 & 1.75 \\
& B & 06 Sep 10 & AH907 & 8.55 \\
\hline \multirow{3}{*}{ IC 10 } & C & 92 Apr 11 & AH453 & 3.7 \\
& D & 97 Nov 10 & AH623 & 4 \\
\hline \multirow{2}{*}{ NGC 3738 } & B & 08 Jan 15, 08 Jan 21, 08 Jan 27 & AH927 & 11.2 \\
& C & 08 Mar 23, 08 Apr 15 & AH927 & 5.85 \\
& D & 08 Jul 8, 08 Jul 24, 08 Jul 25 & AH927 & 0.1 \\
\hline \hline
\end{tabular}




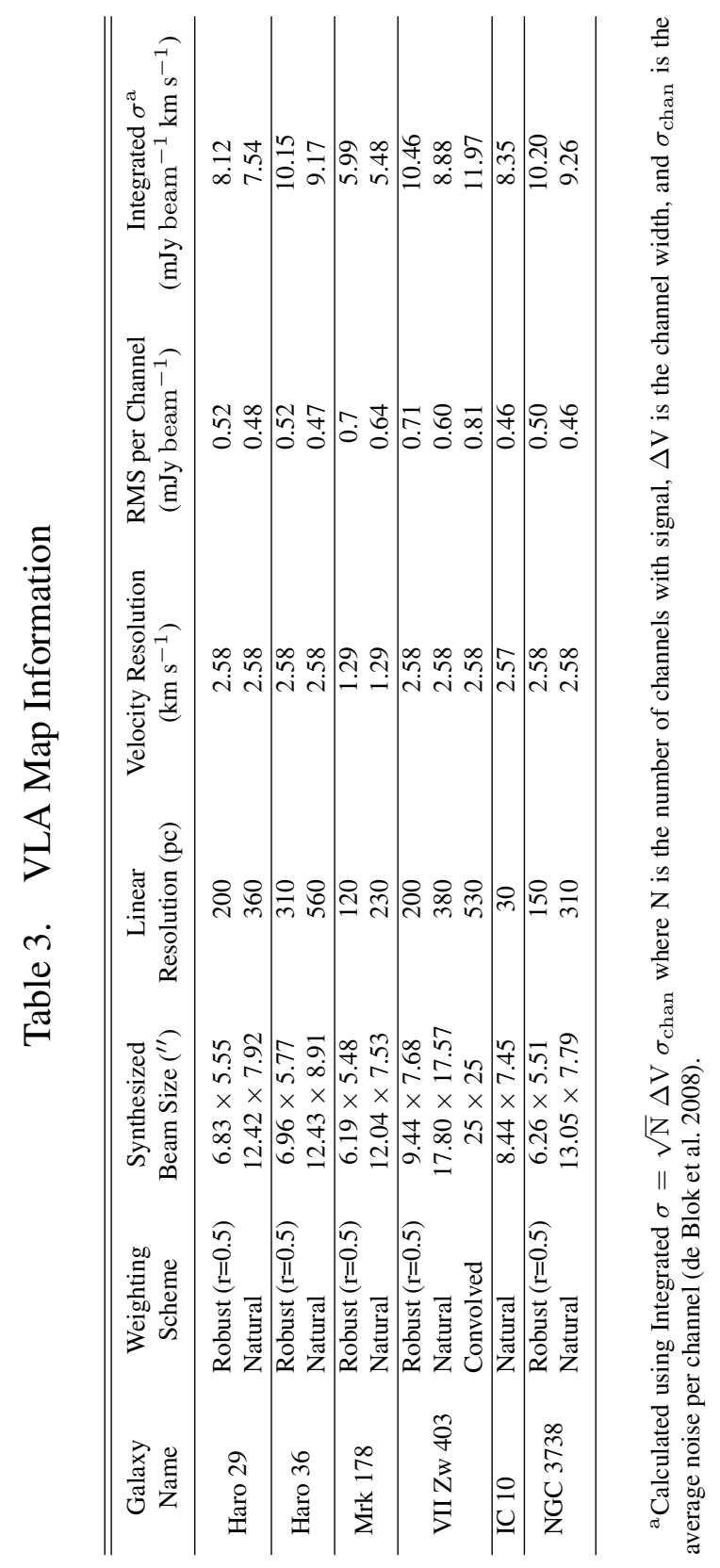

I 


\subsection{The Green Bank Telescope}

The BCD sample in this dissertation was also observed for a total of 203 hours with the GBT (proposal ID GBT/13A-430). For basic GBT observing information, see Table 4. These data were combined with existing GBT data of each galaxy that covered a $2^{\circ} \times 2^{\circ}$ field around each galaxy (Proposal ID GBT/12B-312; P.I. Johnson). The GBT is a single dish radio telescope with a diameter of 100 meters, making it the world's largest fully steerable telescope. However the GBT is small in size when compared to the synthesized telescope of the VLA, therefore, its beam is larger than the synthesized beam of the VLA. The GBT is therefore capable of higher sensitivity measurements at a lower resolution than the VLA.

A technique called "on-the-fly" mapping was implemented to collect the data efficiently; the map was made by scanning across the sky in a raster pattern ${ }^{11}$. The GBT spectra were sampled at the Nyquist rate (for details on Nyquist sampling see Appendix 5). Each galaxy field was approximately $200 \times 200 \mathrm{kpc}^{2}$ in size (at the distance of the galaxy), so that any nearby companions would be found (Taylor et al. 1995; Pustilnik et al. 2001; Thilker et al. 2004; Westmeier et al. 2008; Chynoweth et al. 2011). An exception to this was IC 10 ; IC 10 is only $0.7 \mathrm{Mpc}$ away, therefore a $200 \times 200 \mathrm{kpc}^{2}$ region would cover a $16^{\circ} \times 16^{\circ}$ region of the sky. A region this size would take too long to map at our desired sensitivity. Instead a $2^{\circ} \times 4^{\circ}$ field was mapped for IC 10 . The longer side of IC 10 's map was chosen to show any possible emission extending along the north-south direction. The north-south direction was chosen in order to look for extended emission associated with the southern plume in the VLA integrated H I intensity maps (see section 12 for

\footnotetext{
${ }^{11}$ The raster scan pattern consists of dividing the area of sky to be mapped into rows. The observations are then taken by scanning horizontally one row at a time, and moving vertically after a row of horizontal observations is complete to begin the next horizontal row. This continues until the entire vertical length of the desired region has been mapped.
} 
more details on the VLA H I data). The GBT map for Haro 36 also deviates from the $200 \times 200 \mathrm{kpc}^{2}$ field size. The initial mapping done for this galaxy was a $200 \times 200 \mathrm{kpc}^{2}$ field that had its center shifted up in Galactic Latitude by $\sim 0.23^{\circ}$ to look for evidence of an interaction between Haro 36 and NGC 4707. Then, upon initial mapping of Haro 36's data, it was discovered that the noise in the maps was higher than expected, so extra time was requested and allotted in order to increase the signal-to-noise of the final map. After the full $200 \times 200 \mathrm{kpc}^{2}$ field had been observed several times again, a small amount of the extra time allotted went towards mapping the region below Haro 36 that was missed by shifting the map up in Galactic Latitude, resulting in a $200 \times 240 \mathrm{kpc}^{2}$ field.

Frequency switching was implemented in order to calibrate the data. Frequency switching removes instrumental and atmospheric effects from the data: two observations are made, one with the object of interest at the central frequency of the bandwidth and a second with the central frequency of the bandwidth shifted far enough away from the object of interest that there will be enough blank sky (no emission at those specific frequencies) with which to calibrate the image ${ }^{12}$. In-band frequency switching is when the frequency range centered on the object of interest has some overlap with the second, shifted frequency range, and can be done if the bandwidth of the observations is large enough. The advantage of in-band frequency switching over other observing techniques is that you can always be observing the target source, decreasing the amount of observing time needed. With a $12.5 \mathrm{MHz}$ bandwidth, I was able to use in-band frequency switching with a central frequency switch of $3.5 \mathrm{MHz}$. The data are then initially calibrated and scaled to the temperatures using the following equation:

$$
T_{a}=T_{\text {sys }} \times \frac{o n-o f f}{o f f}
$$

\footnotetext{
${ }^{12}$ Blank sky is used to estimate the zero emission level in the map.
} 
where $T_{\text {sys }}$ is a system temperature that was calculated using a noise diode set at a known temperature readout, and on and off are the readouts when the telescope is on and off frequency (Braatz 2009).

Once collected, the data first have to be corrected for stray radiation, which is emission picked up by the sidelobes of the receiver that appears as real emission (for more information on sidelobes, see Appendix 3). Stray radiation is commonly noticed when the Galactic Plane appears in the frequency range of interest. The Milky Way will not only appear in the main beam, but being a strong, wide angular sized source, it will also show up in the sidelobes. Since the final spectrum does not distinguish between sidelobes and the main beam, this will make the Milky Way spectrum appear wider in frequency and higher in flux than if it was only being picked up by the main beam. The frequency widening can become problematic for later fitting a baseline for removal from the data since there will be fewer zero-level emission channels available for baseline fitting because of channels containing stray Milky Way emission. Stray radiation was a problem for all of my sources. A stray radiation removal program, written by GBT staff, helps reduce the effect of stray radiation by estimating the contribution of the sidelobes based in-part on the date of observations and the location of the Milky Way in the sky. It was run on each spectrum separately so that the correction could be made for each position in the sky. After removing stray radiation, the data were Hanning smoothed in frequency to reduce their noise by approximately one-third (for further details about smoothing, see Appendix 6).

Next, radio frequency interference (RFI) was removed by hand from the data. Radio frequency interference appears as a spike in intensity in the frequency vs. intensity graphs of the data and is caused by manmade objects that emit radio waves in the vicinity of the GBT (e.g., cell phones, microwaves, and wireless internet routers). The frequencies of RFI spikes were recorded for later removal from all spectra in that particular observing session. The RFI spikes were removed by replacing their intensity values with the average 
of the intensity of the frequency channel two channels to the right of an RFI spike and the intensity of the frequency channel two channels to the left of an RFI spike. Boxcar smoothing over three channels at a time was then performed in order to further decrease noise, thereby boosting the signal-to-noise ratio.

The last calibration step taken was fitting a baseline to each spectrum to remove residual instrumental effects. These residual effects appear as low-order polynomials in the frequency vs. intensity graphs of each spectrum and are removed by fitting low-order polynomials to emission-free channels using GBTIDL ${ }^{13}$ (3rd-4th order polynomials were used for the data in this dissertation). The program will take emission-free channels (given by the user) and the polynomial order (also provided by the user) and fit each spectrum individually. Since the purpose of these observations was to look for faint, nearby emission, the emission-free regions for baseline fitting were usually chosen to be far away in frequency/velocity space from the target galaxy because nearby emission associated with the target galaxy will likely have a similar velocity. Baseline subtraction can become challenging if you happen to frequency switch onto another source instead of blank sky. During calibration the off-frequency spectrum is shifted to the on-frequency spectrum's central frequency and subtracted from the on-spectrum. If a source is within $\sim 3.5 \mathrm{MHz}$ of the target's usable frequency range (also $\sim 3.5 \mathrm{MHz}$ in width, therefore the other source can be up to $5.25 \mathrm{MHz}$ away from the central frequency of the target source), then the unwanted source that was originally $1.75-5.25 \mathrm{MHz}$ away from the spectrum, now appears closer in frequency space to the target source as a negative dip in intensity. The unwanted source reduces the amount of frequency space that can be used to estimate the zero-emission baseline for subtraction. NGC 3738's spectra had two sources being

\footnotetext{
${ }^{13}$ GBTIDL is an IDL package written for calibrating and analyzing GBT data. Resources and documentation on GBTIDL can be found at http://gbtidl.nrao.edu/.
} 
reflected into the data. A detailed description of these sources and how they were handled is discussed in Appendix 7.

After the baseline subtraction, the data were then converted to an AIPS-friendly format and read into AIPS for mapping. In AIPS, DBCON was used to combine all of the data sets from different days of observing that corresponded to one galaxy. SDGRD then took the combined data, spatially gridded them, and convolved the data to a spherical Bessel function making a data cube similar to those of the VLA data. The data cube was then integrated along the velocity axis to make a set of maps containing information on the $\mathrm{H}$ I like those of the VLA maps. The GBT H I intensity maps and velocity maps will be discussed throughout this dissertation (the low resolution of the GBT data renders the velocity dispersion maps useless). In order to match the existing data with which my data were combined, my GBT data were taken on a different coordinate system than the VLA data. The different coordinate systems often lead to an orientation difference between the GBT and VLA maps. For basic information on the individual GBT maps, see Table 5. 
Table 4. GBT Observing Information

\begin{tabular}{lcc}
\hline \hline Galaxy Name & Total Time Observing $^{\mathrm{a}}$ (hours) & Angular Size Observed $^{\mathrm{b}}(\mathrm{degr})$ \\
\hline Haro 29 & 25.5 & $2 \times 2$ \\
Haro 36 & 25 & $1.2 \times 1.43$ \\
Mrk 178 & 58.5 & $2.9 \times 2.9$ \\
VII Zw 403 & 43.5 & $2.6 \times 2.6$ \\
IC 10 & 50.5 & $2 \times 4$ \\
NGC 3738 & 34.25 & $2.3 \times 2.3$ \\
\hline
\end{tabular}

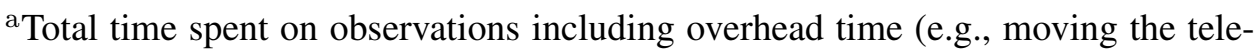
scope, getting set up for observations, and time on calibrators.)

${ }^{b}$ These angular sizes represent the $200 \times 200 \mathrm{kpc}^{2}$ fields of the galaxies with the exceptions of IC 10 and Haro 36. However, for observing purposes these fields had 0.1 degrees added horizontally and vertically to them as a buffer. This was done to account for the time that the telescope would start/stop moving vs. the time that the telescope began recording data. 


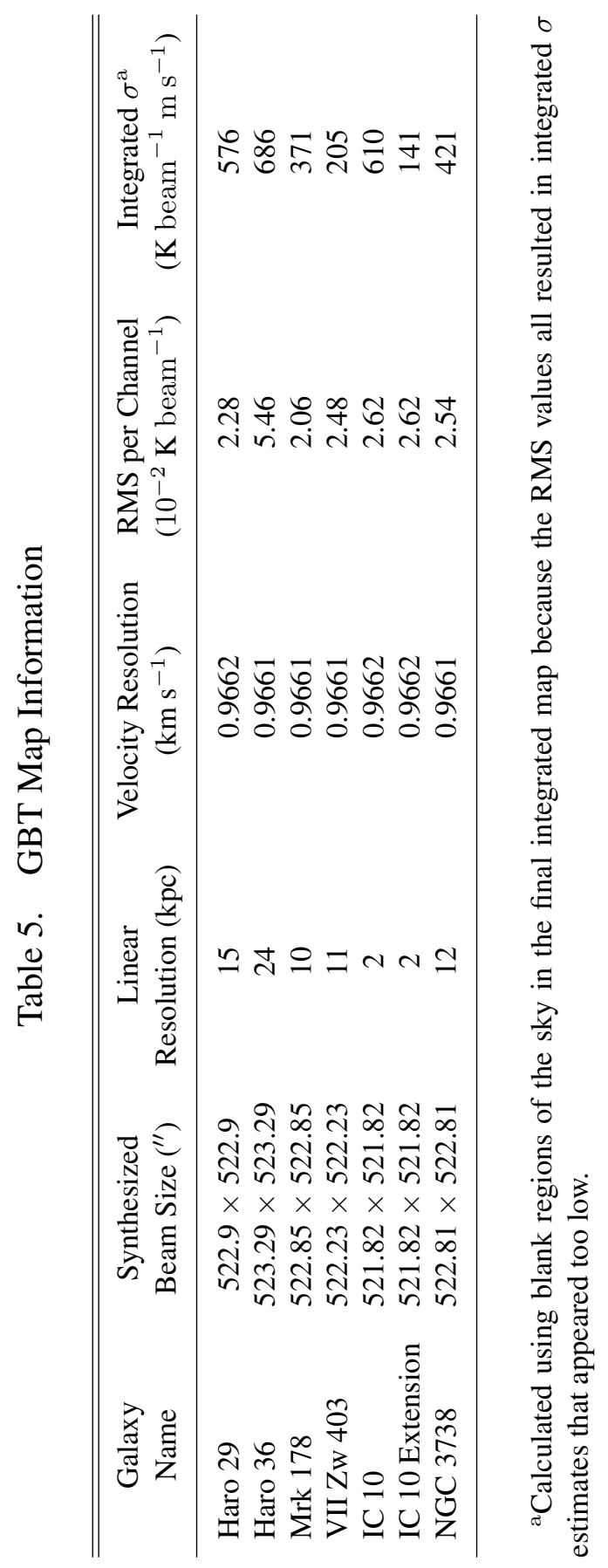


4. Results: Haro 29

\subsection{Stellar Component}

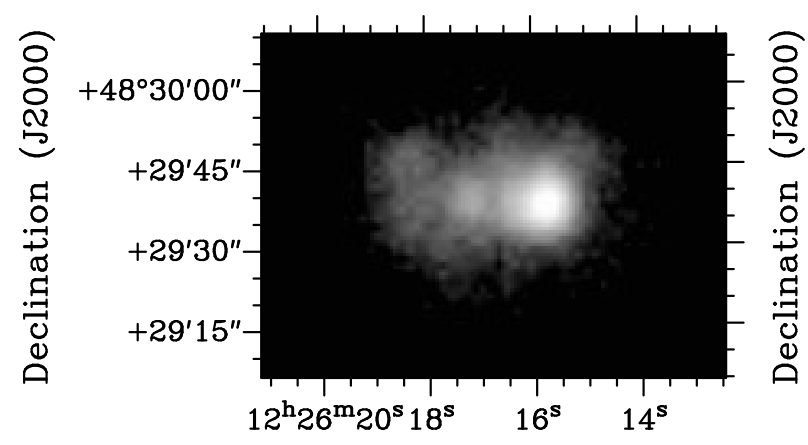

Right Ascension (J2000)

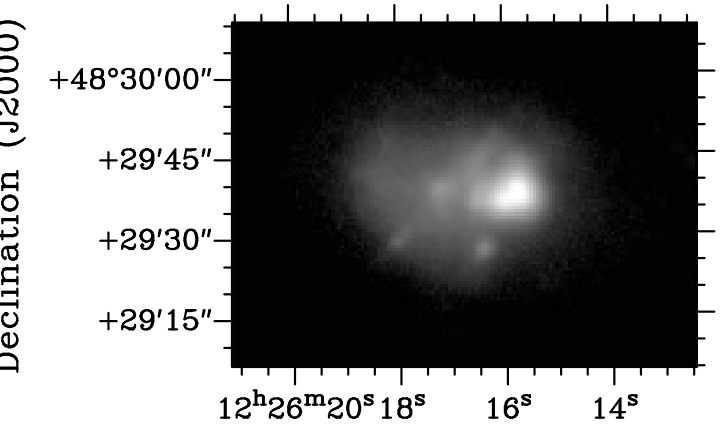

Right Ascension (J2000)

Fig. 1.- Haro 29 Left: FUV.; Right: V-band

Haro 29's FUV and V-band images are shown in Figure 1. The FUV data were taken with Galaxy Evolution Explorer (GALEX) and provide information about the star formation that has occurred over the past 100-200 Myr (Hunter et al. 2010). The V-band data were taken with the Lowell Observatory $1.8 \mathrm{~m}$ Perkins Telescope (Hunter \& Elmegreen 2006) and provide information about the star formation that has taken place over the past Gyr (Hunter \& Elmegreen 2006). Haro 29's FUV and V-band maps show nearly the same morphology.

\subsection{VLA H I Morphology}

Haro 29's natural weighted integrated H I intensity map as measured by the VLA is shown in the top-left of Figure 2. Haro 29's H I morphology can be separated into two parts: the region of high column density and the tenuous, outer emission. The tenuous, outer gas generally sits off to the west (right) of the region of high column density and has two components: one southern extension and a curved extension that begins to the north 

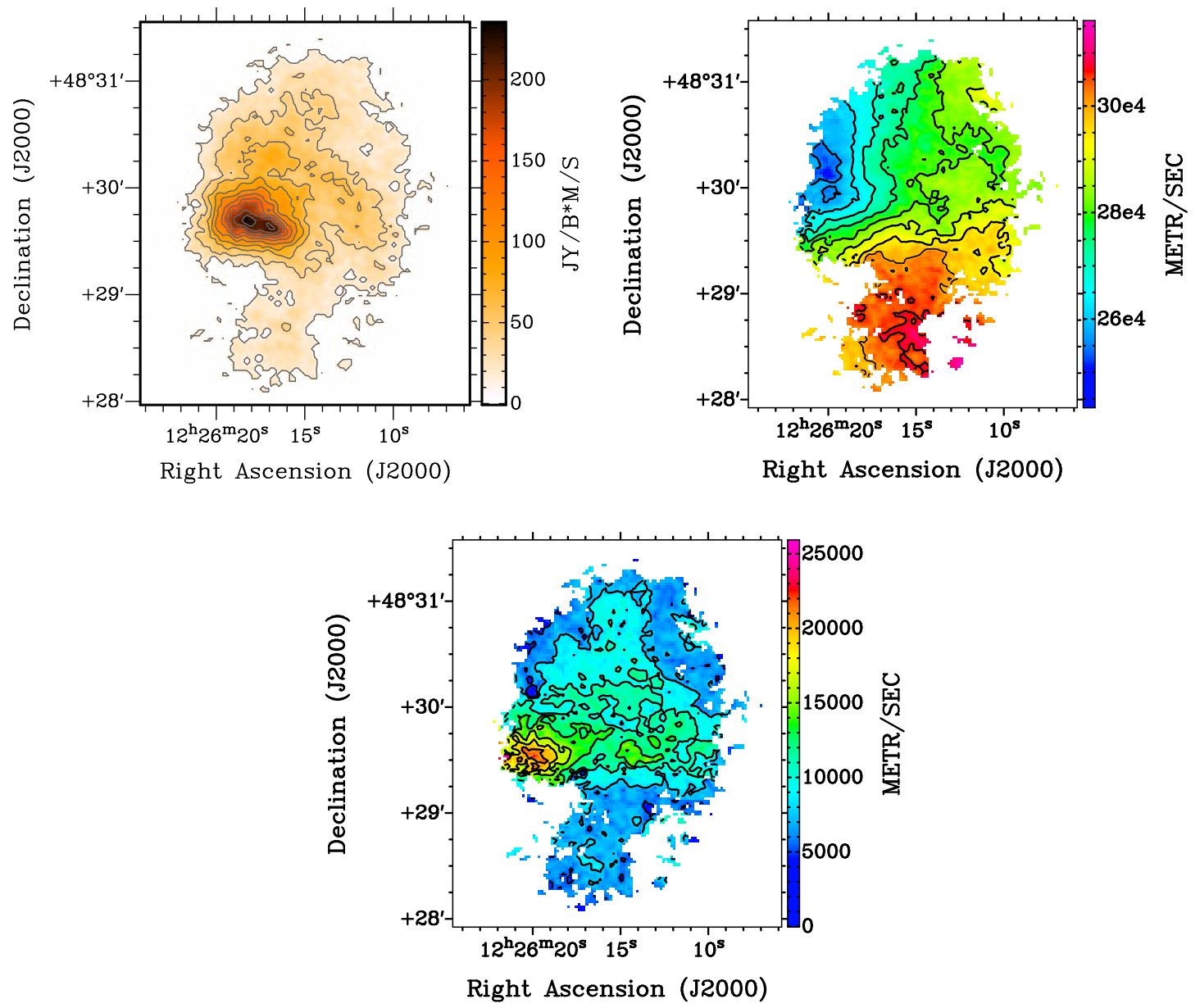

Fig. 2.- Haro 29's integrated H I natural-weighted moment maps. Upper left: Integrated $\mathrm{H}$ I intensity map; contour levels are $1 \sigma \times(2,5,8,11,14,17,20,23,26$, and 29) where $1 \sigma=8.47 \times 10^{19}$ atoms $\mathrm{cm}^{-2}$. Upper right: Velocity field; contour levels are $256.25 \mathrm{~km}$ $\mathrm{s}^{-1}$ to $306.25 \mathrm{~km} \mathrm{~s}^{-1}$ separated by $6.25 \mathrm{~km} \mathrm{~s}^{-1}$. Bottom: Velocity dispersion field; contour levels are 5.18 to $20.72 \mathrm{~km} \mathrm{~s}^{-1}$ separated by $2.59 \mathrm{~km} \mathrm{~s}^{-1}$.

of the region of high column density, curves to the west, and then down to the south. The region of high column density has two central H I peaks.

Haro 29's robust weighted H I map, shown in the top-left of Figure 3, is higher resolution and lower sensitivity than the natural weighted map. In the robust weighted map, the two central peaks in the region of high column density become more defined. The projected distance between the two central peaks is measured to be $\sim 500 \mathrm{pc}$ by taking 

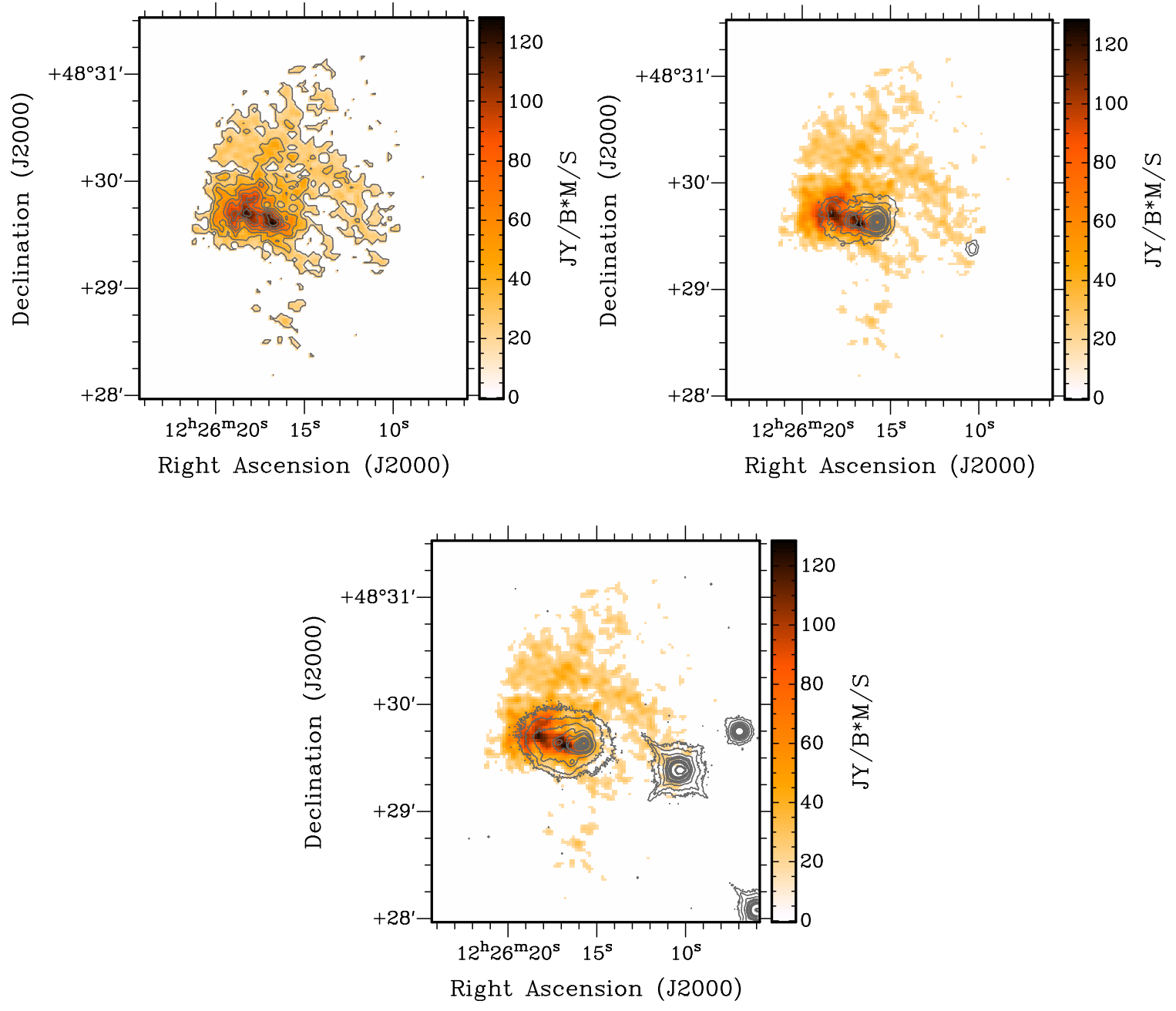

Fig. 3.- Haro 29's integrated H I robust-weighted moment maps. Upper left: Integrated $\mathrm{H}$ I intensity map; contour levels are $1 \sigma \times(2,4,6,8,10,12,14)$ where $1 \sigma=2.37 \times$ $10^{20}$ atoms $\mathrm{cm}^{-2}$. Upper right: Integrated $\mathrm{H} \mathrm{I}$ intensity map colorscale and FUV contours. The single FUV source to the west of the other contours is a foreground star. Bottom: Integrated $\mathrm{H}$ I intensity map colorscale and V-band contours. The large sources to the west of the main V-band emission are foreground stars.

the distance between the two brightest pixels in each peak in the robust weighted map. The FUV and V-band contours have been plotted over the colorscale of the H I column density, robust weighted map in order to compare the stellar and gaseous components of Haro 29, in the top-right and bottom of Figure 3 respectively. The most luminous stellar 
components of both the FUV and V-band data are slightly offset to the west of the peaks in the highest density region.

\subsection{VLA H I Velocity and Velocity Dispersion Field}

Haro 29's VLA H I velocity field is shown in the top-right of Figure 2. The region of high $\mathrm{H}$ I column density has nearly parallel isovelocity contours, indicating that this region is participating in solid-body rotation with a kinematic major axis at a position angle (PA) of roughly $30-35^{\circ}$ (estimated by eye). The tenuous $\mathrm{H} \mathrm{I}$ emission just to the north of the dense $\mathrm{H} \mathrm{I}$ is rotating on a different kinematic major axis with a kinematic major axis of roughly $90^{\circ}$ (estimated by eye).

The $\mathrm{H}$ I velocity dispersion field as measured by the VLA is located at the bottom of Figure 2. The velocity dispersions reach nearly $20 \mathrm{~km} \mathrm{~s}^{-1}$ in the area southeast of the high $\mathrm{H} \mathrm{I}$ density region. The outer, tenuous gas has velocity dispersions of $10 \mathrm{~km} \mathrm{~s}^{-1}$ and below.

\subsection{GBT H I Morphology and Velocity Field}

Haro 29's integrated H I intensity map as measured with the GBT is shown in the left side of Figure 4. The GBT H I map is at a much higher sensitivity and lower resolution than the VLA maps (the VLA maps would fit in approximately one pixel of the GBT map). Haro 29 is the circular emission at the center of the map. The small pieces of emission surrounding Haro 29 are from noise in the map. At the sensitivity of the map, there are no

other H I objects in the region. The velocity field of the GBT H I data is shown in the right side of Figure 4. The velocity field map has been blanked to include only the velocities of Haro 29 at $5 \sigma$ and above (noise in the maps also have calculated velocities which can 

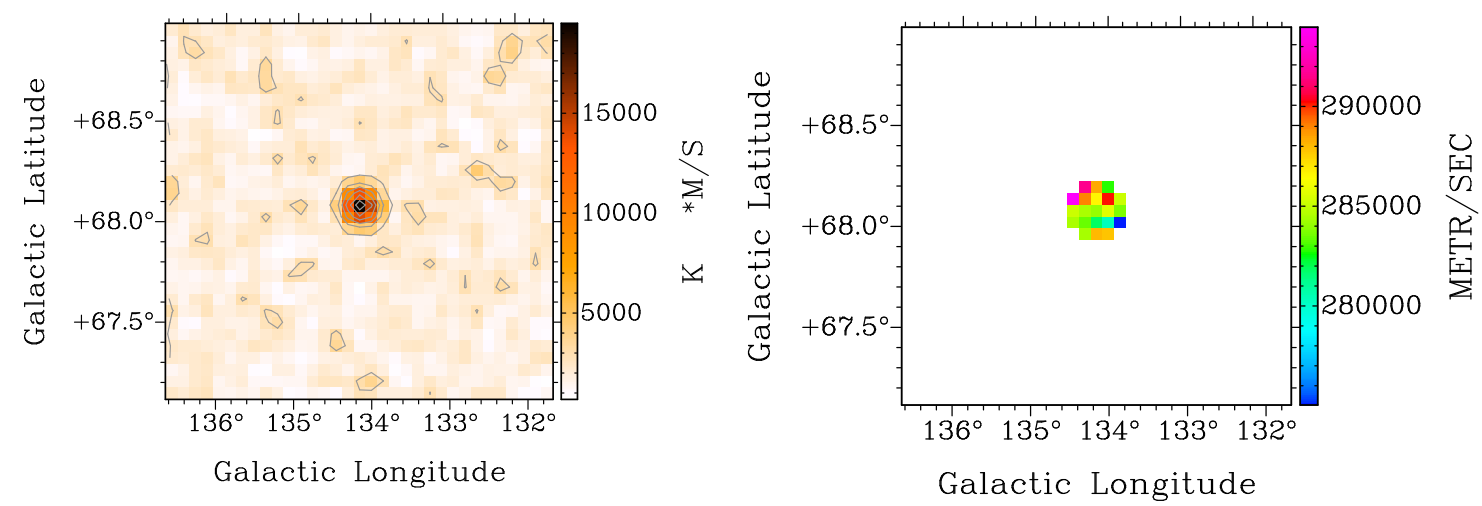

Fig. 4.- Haro 29 Left: GBT H I column density; contour levels are $1 \sigma \times(5,10,15,20,25$, 30) where $1 \sigma=4.48 \times 10^{17}$ atoms $\mathrm{cm}^{-2}$. Right: The blanked GBT H I velocity field.

make velocity maps look confusing if blanking is not done). There is a negative velocity gradient in Haro 29 from top to bottom.

\subsection{H I Mass}

The H I masses (in units of solar masses) for the VLA data were calculated using the following equation:

$$
M\left(M_{\odot}\right)=235.6 D^{2} \sum_{i} S_{i} \Delta V
$$

where $D$ is the distance of the galaxy in units of $\mathrm{Mpc}, S_{i}$ is the flux in $\mathrm{mJy}^{14}$ in channel $\mathrm{i}$, and $\Delta V$ is the channel width in $\mathrm{km} \mathrm{s}^{-1}$. In all of my data sets $\Delta \mathrm{V}$ is a constant. The sum of the flux is determined using the task ISPEC or the task BLSUM in AIPS. The task ISPEC allows the user to define a box in the spatial plane of the galaxy image. Within the boxed region ISPEC sums the flux for the user-specified velocity channel range to give a total flux in units of Jy. The same process is completed with BLSUM, however, the user is able

\footnotetext{
${ }^{14} \mathrm{Jansky}(\mathrm{Jy})$ is a unit of flux commonly used in radio astronomy. $1 \mathrm{Jy}=10^{-26} \mathrm{~W} \mathrm{~m}^{-2} \mathrm{~Hz}^{-1}$
} 
to define a region of any shape for the flux summation by tracing it out on a map of the galaxy.

The H I masses of the GBT data are given by a different equation; the units of the GBT data are given in temperature brightness or Kelvin, as opposed to Jy. Using the following conversion:

$$
S=1.65 \times 10^{-3} \Delta \alpha \Delta \delta T
$$

(private communications, E. Brinks) where flux is given in mJy, $\alpha$ is the Full-Width Half-Maximum (FWHM) of the major axis of the beam in arcsec, $\delta$ is the FWHM of the minor axis of the beam in arcsec, and $\mathrm{T}$ is the temperature in Kelvin, we can substitute the flux in equation 2 to get:

$$
M\left(M_{\odot}\right)=1.2 \times 10^{5} D^{2} \sum_{i} T_{i} \Delta V
$$

where FWHM of the beam is $\sim 9^{\prime}$ or $\sim 540^{\prime \prime}$. The same methods as those used for the VLA data can then be used to get the sum of the temperature from the GBT data. However, the ISPEC output will be given in units of Kelvin/(beam pixel $\left.{ }^{-1}\right)$, therefore the following equation was used to convert the output into units of Kelvin:

$$
\sum_{i} T_{i}=T_{\mathrm{ISPEC}} \frac{\Delta x \Delta y / \text { pixel }}{1.13 \Delta \alpha \Delta \delta / \text { beam }}
$$

(private communications, E. Brinks) where $T_{\text {ISPEC }}$ is the output from ISPEC and $\Delta \mathrm{x}$ and $\Delta y$ are the dimensions of the pixel sizes given in arcseconds. The 1.13 comes from the geometric difference between a gaussian beam and a square pixel.

Haro 29's mass from the GBT is $7.4 \times 10^{7} M_{\odot}$ and from the VLA is $5.8 \times 10^{7} M_{\odot}$, giving the VLA data $78 \%$ the mass of the GBT data. From the VLA data, the mass of the inner, dense $\mathrm{H}$ I emission was measured using BLSUM to be $1.4 \times 10^{7} M_{\odot}$ or $24 \%$ 
of the total VLA H I mass. The inner region was chosen to be the $\mathrm{H}$ I within the contour that is fourth from the bottom in the natural weighted map at $9.32 \times 10^{20}$ atoms $\mathrm{cm}^{-2}$. The $9.32 \times 10^{20}$ atoms $\mathrm{cm}^{-2}$ contour was chosen because it is the lowest contour that does not appear to have any curved features of the outer, tenuous gas. This means that the outer region composes $76 \%$ of Haro 29's total H I mass from the VLA data.

\section{Discussion: Haro 29}

Haro 29 is a morphologically and kinematically disturbed galaxy. Haro 29's region of high H I column density has solid body rotation. The outer, tenuous gas has a curved overall shape and is also rotating about a different axis than the dense $\mathrm{H}$ I region. The outer, tenuous gas also has low velocity dispersion of $\sim 10 \mathrm{~km} \mathrm{~s}^{-1}$, which is typical for an undisturbed dwarf irregular galaxy (Tamburro et al. 2009; Warren et al. 2012). The dense $\mathrm{H}$ I region appears to have more disturbed gas with dispersions of $\sim 20 \mathrm{~km} \mathrm{~s}^{-1}$.

The GBT data do not show any possible companions in the vicinity at the sensitivity of the map, therefore Haro 29 is unlikely to have recently interacted with a companion galaxy. It is possible that Haro 29 is an advanced merger. If the two galaxies that merged to form Haro 29 were not rotating in the same direction, then the remnants of these two components may be discernible through a detailed study of the gas kinematics. The two central $\mathrm{H}$ I peaks in the region of high $\mathrm{H}$ I column density, for example, could be the two cores of galaxies that have merged and may still be kinematically distinct. However, if the gas components were rotating in the same direction or the merger is so advanced that the gas has had time to settle into a regular rotation pattern, then it may not be possible to distinguish the two components.

One way to distinguish gas that is rotating against a galaxy's rotation pattern is to use position-velocity $(\mathrm{P}-\mathrm{V})$ diagrams. Position-velocity diagrams are made by placing a line 

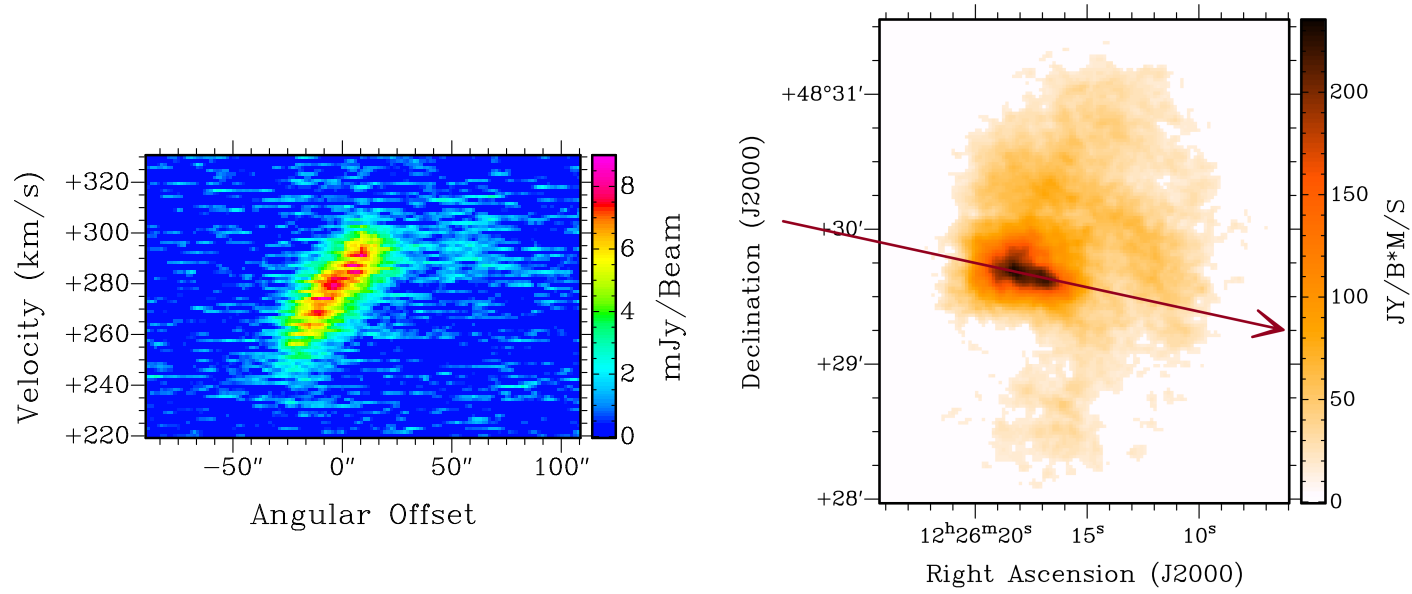

Fig. 5.- Haro 29: The left column contains the P-V diagram and the right column contains the natural-weighted integrated $\mathrm{H}$ I intensity map with a red arrow indicating the location of the corresponding slice through the galaxy and pointing in the direction of positive offset. The slice is taken to go through both central peaks.

(a.k.a. a slice) on an image of the galaxy. The position along the slice is then plotted vs. all the velocities in the data cube. The intensity of the emission with a specific velocity and position along the slice is then represented in the $\mathrm{P}-\mathrm{V}$ diagram as a colorscale. Karma is an interactive program used for this work that produces P-V diagrams for user-drawn slices. The slice in Karma can be freely rotated about the center by the user, placed anywhere over the galaxy, and redrawn with an automatic update of the P-V diagram. A P-V diagram for Haro 29's two central H I peaks is shown in Figure 5. On the left side is the P-V diagram and on the right side is the slice used for the P-V diagram (the red arrow) plotted on top of Haro 29's integrated H I intensity. The arrow's direction represents the direction of increasing angular offset towards positive offsets on the P-V diagram.

The P-V diagram shows that the two central peaks are rotating together in a solid body fashion and are not kinematically distinct from each other. Their solid body like rotation can be seen in the $\mathrm{P}-\mathrm{V}$ diagram as they smoothly transition into each other with constantly increasing velocities towards higher offsets. The two $\mathrm{H}$ I peaks may be the result of lumpy gas morphology or the stellar population affecting the gas through 
consumption, ionization, stellar winds, and supernovae. Schwartz et al. (2006) found that the brightest stellar regions of Haro 29 were associated with large ionized gas outflow speeds of $-191 \pm 92 \mathrm{~km} \mathrm{~s}^{-1}$. These outflow speeds are from the movement of the warm and cold gas surrounding the stellar population, as measured by the Doppler shift absorption features in the ultraviolet. These large outflows indicate that the stellar population may be having a large effect on the surrounding gas, perhaps helping to form the two central peaks in the $\mathrm{H}$ I.

The rest of the $\mathrm{H}$ I emission in Haro 29 was checked for kinematic peculiarities using P-V diagrams, but no new features were found. These results, however, do not mean that Haro 29 is not a merger, only that if it is a merger, then the gas has probably had time to settle into a regular rotation pattern in the dense $\mathrm{H}$ I inner region. The two different kinematic axes may be a result of the outer gas still being in the process of settling into orbit with the rest of the gas.

Haro 29 may have also recently accreted the outer gas. Accretion of gas could explain the two different kinematic major axes. The mass of the outer tenuous gas, however, is $6 \times 10^{7} M_{\odot}$ or $76 \%$ of Haro 29's total VLA H I mass, which is a large amount of gas to simply accrete from the intergalactic medium (IGM). Sancisi et al. (2008) state that currently no starless $\mathrm{H}$ I clouds have been detected with masses above $\sim 10^{7} M_{\odot}$ in galaxy group surveys. Therefore, if the outer H I is from accretion of the IGM, then it would have been a very large amount of gas that was accreted or several gas clouds combined to make up the outer tenuous region.

Haro 29's lopsided morphology, with the dense H I region offset to the east and the tenuous gas to the west, could also be a result of ram pressure stripping ${ }^{15}$. If Haro 29 is

\footnotetext{
${ }^{15}$ Ram pressure stripping is when a galaxy moving through dense IGM has its own gas stripped by the impact of the IGM.
} 
experiencing ram pressure stripping, then the leading edge of the galaxy moving through the IGM would be the dense $\mathrm{H}$ I region, and the trailing end of the galaxy would be the tenuous gas. However, the curved appearance of the northwestern, tenuous gas is difficult to explain in this case; ram pressure stripping would likely leave straight tails of gas behind the galaxy. Ram pressure stripping also usually occurs in regions where the density of galaxies is high since high galaxy density suggests larger amounts of IGM, but Haro 29 is part of the Canis Venatici Group, a low galaxy density group. It is therefore unlikely that Haro 29 is experiencing ram pressure stripping, leaving the most likely scenario of Haro 29 being an advanced merger, where the two peaks may be a result of the merger or the result of star formation. 
6. Results: Haro 36

\subsection{Stellar Component}
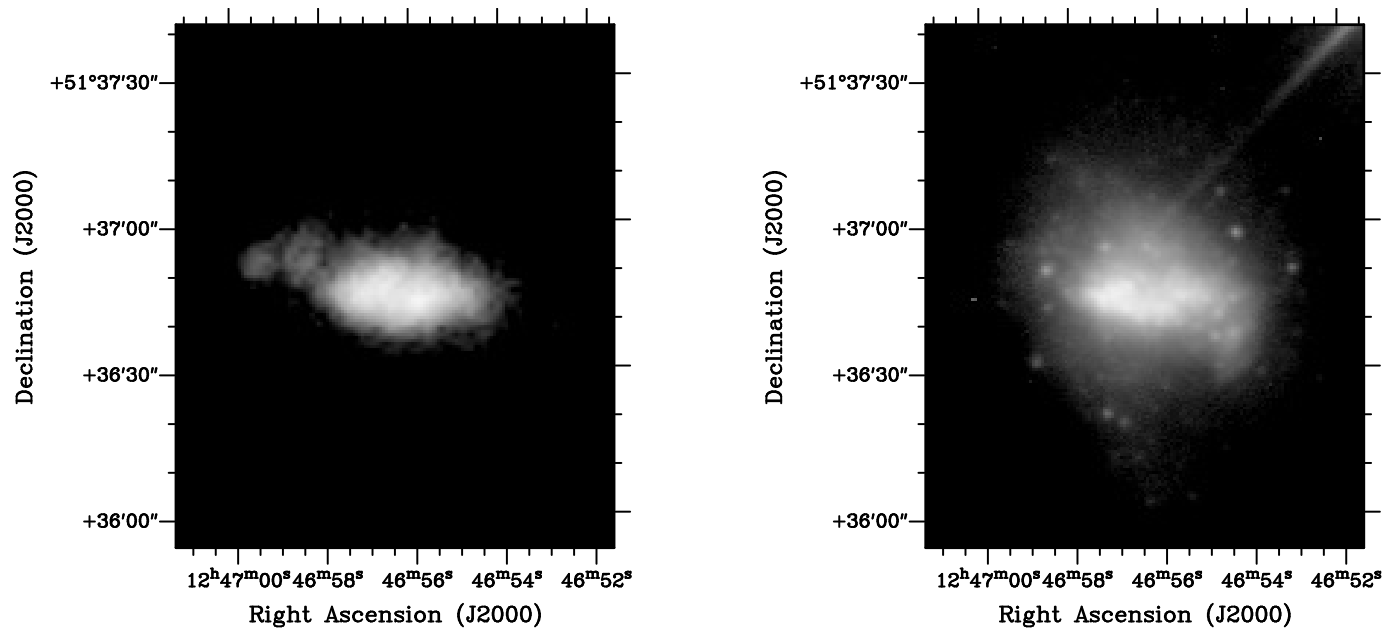

Fig. 6.- Haro 36 Left: FUV.; Right: V-band; the streak coming from the top-right of the image is due to a foreground star in the image.

Haro 36's V-band and FUV images are shown in Figure 6. The FUV data were taken with GALEX and the V-band data were taken with the Lowell Observatory 1.8m Perkins Telescope. Both of these images have a similar inner morphology, however, the V-band image shows an extended disk in the north-south direction. The extended disk in the V-band led Hunter \& Elmegreen (2006) to define the major axis of Haro 36's stellar disk as having a PA of $1.2^{\circ}$ with an inner stellar bar at the PA of $76^{\circ}$.

\subsection{VLA H I Morphology}

The integrated $\mathrm{H}$ I intensity map of the natural weighted data is shown in the top-left of Figure 7. This map has two distinct H I peaks. The galaxy appears to have a disk-like morphology with an added extension in the north from $51^{\circ} 37^{\prime} 15^{\prime \prime}$ to $51^{\circ} 38^{\prime} 15^{\prime \prime}$. 

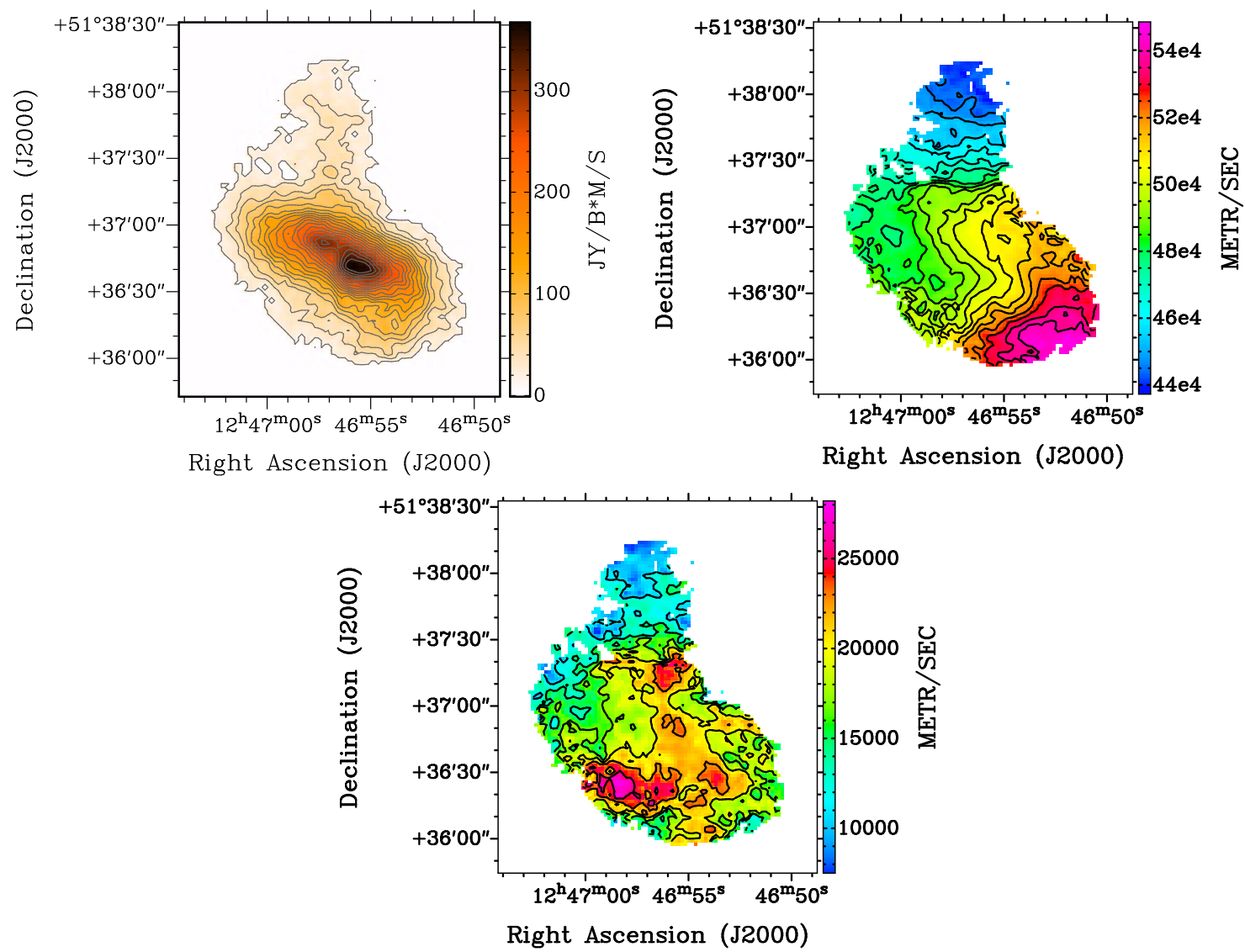

Fig. 7.- Haro 36's integrated H I natural-weighted moment maps. Upper left: Integrated H I intensity map; contour levels are $1 \sigma \times(2,4,6,8,10,12,14,16,18,20,22,24,26,28$, $30,32,34,36,38)$ where $1 \sigma=9.15 \times 10^{19}$ atoms $\mathrm{cm}^{-2}$. Upper right: Intensity weighted velocity field; contour levels are $445.5 \mathrm{~km} \mathrm{~s}^{-1}$ to $539 \mathrm{~km} \mathrm{~s}^{-1}$ separated by $5.5 \mathrm{~km} \mathrm{~s}^{-1}$. Bottom: Velocity dispersion field; contour levels are 11.28 to $25.38 \mathrm{~km} \mathrm{~s}^{-1}$ at intervals of $2.82 \mathrm{~km} \mathrm{~s}^{-1}$.

The integrated H I intensity map of the robust weighted data is in the top-left of Figure 8. This map shows the two central peaks again, and some emission from the northern extension. The centers of the two central peaks are separated by a projected distance of $\sim 700 \mathrm{pc}$. The separation between the two peaks was measured in the robust map by taking the projected distance between the brightest pixels in the peaks. The V-band and FUV contours have been plotted over the colorscale of the robust weighted, H I intensity map in the top-right and bottom (respectively) of Figure 8. Most of the ongoing 

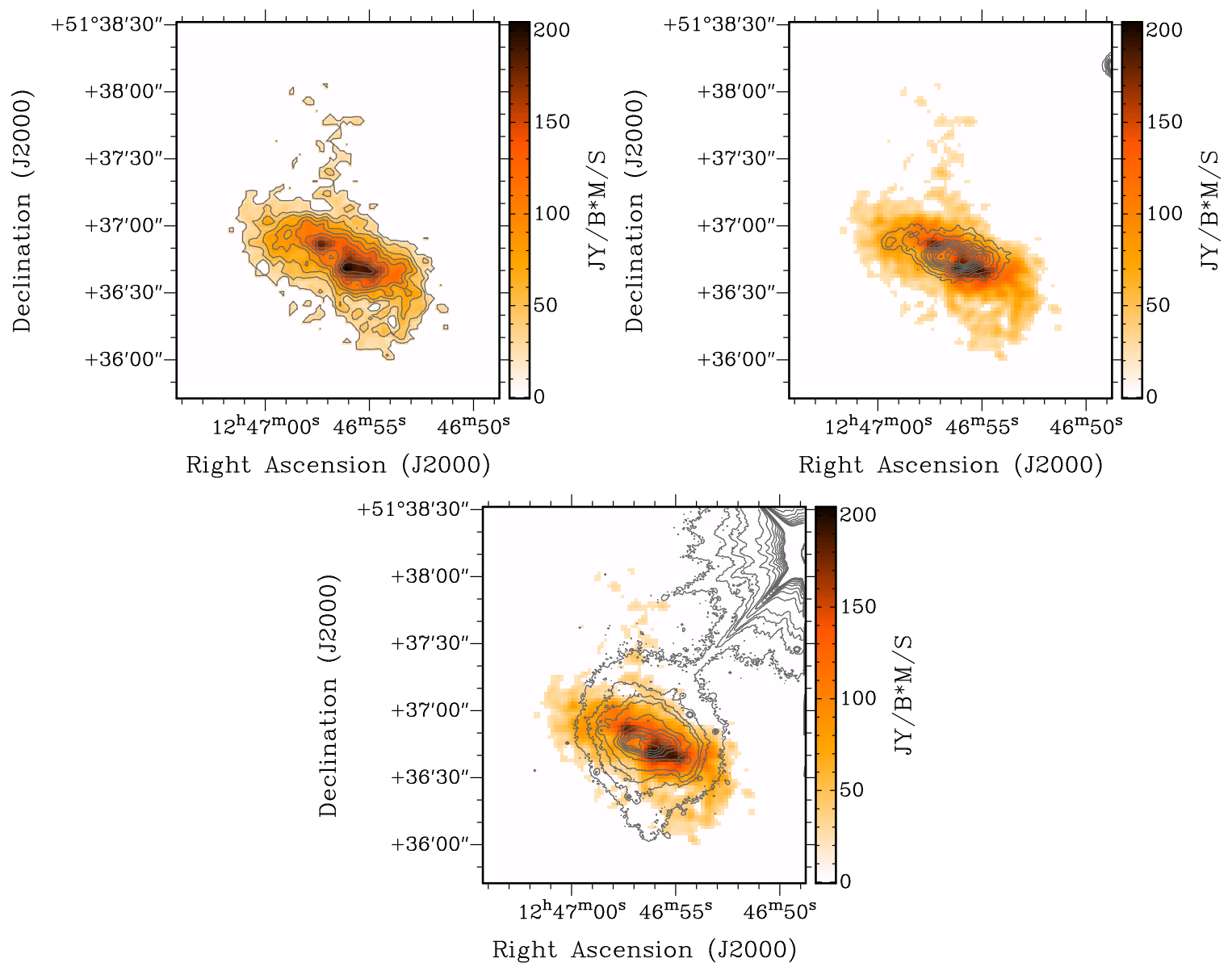

Fig. 8.- Haro 36's integrated H I robust-weighted moment maps. Upper left: Integrated $\mathrm{H}$ I intensity map; contour levels are $1 \sigma \times(2,4,6,8,10,12,14,16,18)$ where $1 \sigma=$ $2.79 \times 10^{20}$ atoms $\mathrm{cm}^{-2}$. Upper right: Integrated $\mathrm{H}$ I intensity map colorscale and FUV contours. Bottom: Integrated H I intensity map colorscale and V-band contours.

star formation (FUV) and previous star formation (V-band) has, in projection, occurred on and between the two central $\mathrm{H}$ I peaks.

\subsection{VLA H I Velocity and Velocity Dispersion Field}

The H I velocity field of Haro 36's VLA data is shown in the top-right of Figure 7. The velocity field across the main body is remniscent of solid body rotation, but there are many large kinks in the isovelocity contours. Interestingly, the isovelocity contours 
of the northern extension are nearly perpendicular to the isovelocity contours in the main body. The northern extension has a projected length of $2.2 \mathrm{kpc}$. The length of the northern extension was calculated in the natural weighted map from the northern tip of the $2 \sigma \mathrm{H} \mathrm{I}$ column density contour to the approximate center of the $473 \mathrm{~km} \mathrm{~s}^{-1}$ isovelocity contour. The $473 \mathrm{~km} \mathrm{~s}^{-1}$ contour was chosen because it is the most southern contour that appears related to the kinematics of the northern extension, in that it is nearly perpendicular to the isovelocity contours seen in the main body.

The H I velocity dispersion of Haro 36's VLA data is shown in the bottom of Figure 7. A significant amount of the main body has velocity dispersions near $20 \mathrm{~km} \mathrm{~s}^{-1}$, while the northern extension appears to have low velocity dispersions around $10 \mathrm{~km} \mathrm{~s}^{-1}$.

\subsection{GBT H I Morphology and Velocity Field}

Haro 36's integrated H I intensity map as measured with the GBTis shown in the left side of Figure 9. Haro 36 appears at a galactic latitude of $65.5^{\circ}$. A second galaxy, NGC 4707 , appears at a galactic latitude of almost $66^{\circ}$. There does not appear to be any physical $\mathrm{H}$ I connection between the two galaxies at the sensitivity of the map. The blanked GBT velocity field in the right of Figure 9 shows a small gradient across both galaxies.

\subsection{H I Mass}

Haro 36's total H I mass detected by the VLA is $1.3 \times 10^{8} M_{\odot}$ and the total H I mass detected by the GBT is $1.9 \times 10^{8} M_{\odot}$. The VLA recovered $68 \%$ of the GBT mass. From the natural weighted VLA H I data, the masses of individual features were obtained using BLSUM. The masses of the two peaks were calculated in the areas defined by the $\mathrm{H}$ I column density contour of $2.9 \times 10^{21}$ atoms $\mathrm{cm}^{-2}$ in the natural weighted map. The 

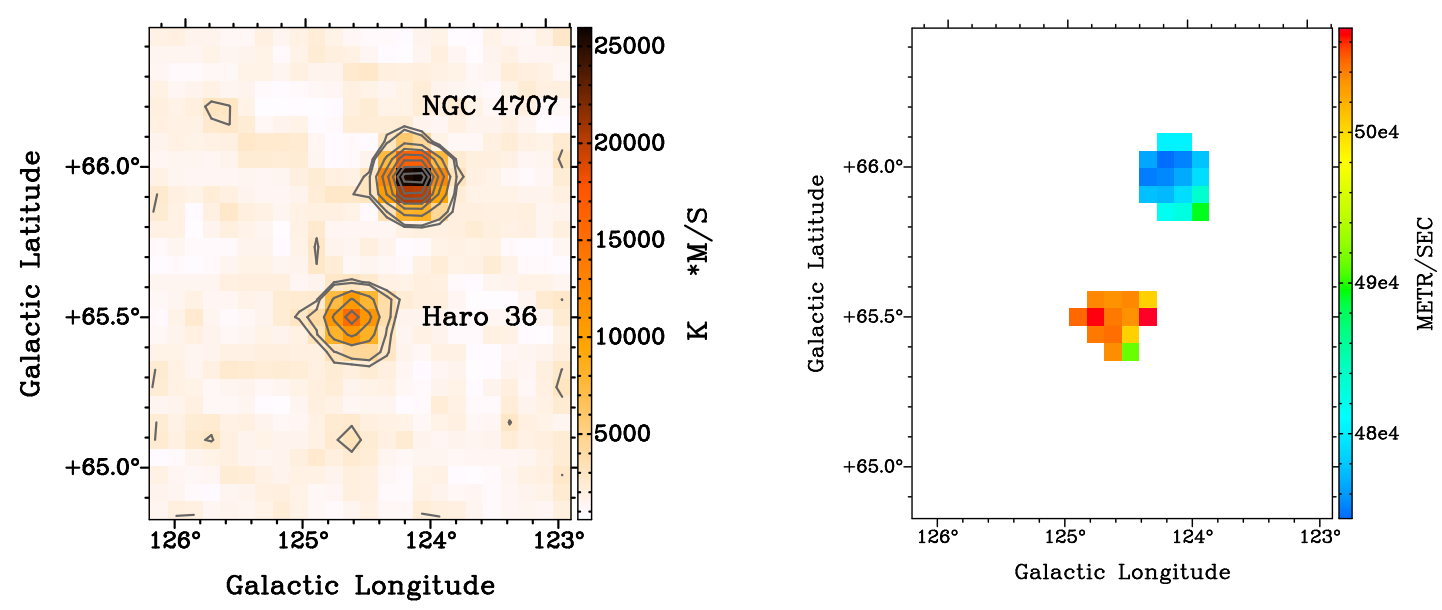

Fig. 9.- Haro 36 Left: GBT H I column density; contour levels are $1 \sigma \times(4,5,10,15,20$, $25,30,35)$ where $1 \sigma=5.34 \times 10^{17}$ atoms $\mathrm{cm}^{-2}$. Right: GBT H I velocity field.

$2.9 \times 10^{21}$ atoms $\mathrm{cm}^{-2}$ contour was used because it is the lowest level contour where the peaks are still separate. The peak to the northeast has a mass of $2.0 \times 10^{6} M_{\odot}$ and the peak to the southwest has a mass of $1.1 \times 10^{7} M_{\odot}$. Therefore the $\mathrm{H}$ I mass along the line of sight of the northeast peak is $18 \%$ of the mass along the line of sight of the southwest peak. The northern extension's mass was calculated to be $10^{7} M_{\odot}$ or $\sim 8 \%$ of the total mass of Haro 36 , the $473 \mathrm{~km} \mathrm{~s}^{-1}$ contour again served as a border for the northern extension.

\section{Discussion: Haro 36}

The two central H I peaks in the main body of Haro 36 may have been created by the star formation that is occurring between them. The gas between the two peaks could once have had a higher column density, and the stellar population may then have consumed the gas during formation, and produced stellar winds to blow out even more gas from the region between them. If the star formation took place slowly, over a long period of time, then the gas between the peaks may have been consumed without having too much of an effect on the kinematics of the surrounding gas. However, if the star formation was 
vigorous and dense, the surrounding gas kinematics have likely been disturbed by the stellar component, creating a hole between the two H I peaks.

To see how the kinematics of these two gas clouds appear with respect to each other, a $\mathrm{P}-\mathrm{V}$ diagram was made by taking a slice through the approximate centers of each peak. The P-V diagram through both peaks and its respective slice are shown in the top row of Figure 10. The gas appears to be generally increasing in velocity with increasing angular offset in a solid body pattern. The overall appearance of the P-V diagram resembles a bird's eye view of a mouse; the ear of the mouse on the left side of the diagram is the northeast peak and the ear of the mouse on the right side of the diagram is the southwest peak, the pointed nose of the mouse is to the bottom-left of the P-V diagram, and the tail of the mouse is to the top-right of the P-V diagram. The velocities in the line of sight of the southwest peak cover a larger velocity range than those in the line of sight of the northeast peak; the southwest peak covers $\sim 460-540 \mathrm{~km} \mathrm{~s}^{-1}$, while the northeast peak covers $\sim 470-520 \mathrm{~km} \mathrm{~s}^{-1}$. The southwest peak is centered in the P-V diagram at $\sim 485 \mathrm{~km}$ $\mathrm{s}^{-1}$ and $\sim 8^{\prime \prime}$ and is offset from the general solid body motion of the rest of the main body. The northeast peak is centered at $505 \mathrm{~km} \mathrm{~s}^{-1}$ and about $-8^{\prime \prime}$ and appears to be rotating with the rest of the main body. It is possible that the southeast peak has been highly kinematically disturbed by stellar winds and supernovae explosions pushing the gas in the peak, but there is no apparent $\mathrm{H}$ I hole between the peaks in the mouse P-V diagram. If there is a hole, then it may be unresolved. It is also possible that one of the two $\mathrm{H}$ I peaks is an external gas cloud in the line of sight of the disk of the galaxy. If one of the peaks is an external gas cloud, then the gas cloud could even be impacting the disk, causing star formation to occur between the two gas clouds.

A P-V diagram at the angle of the major axis of the stellar disk was also made, and is shown in the second row of Figure 10. The P-V diagram through the stellar major axis happens to pass through the northern extension, which appears in the bottom-left of 


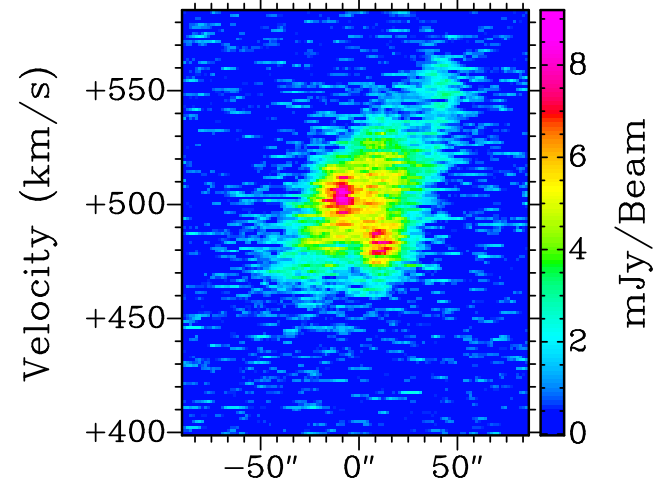

Angular Offset

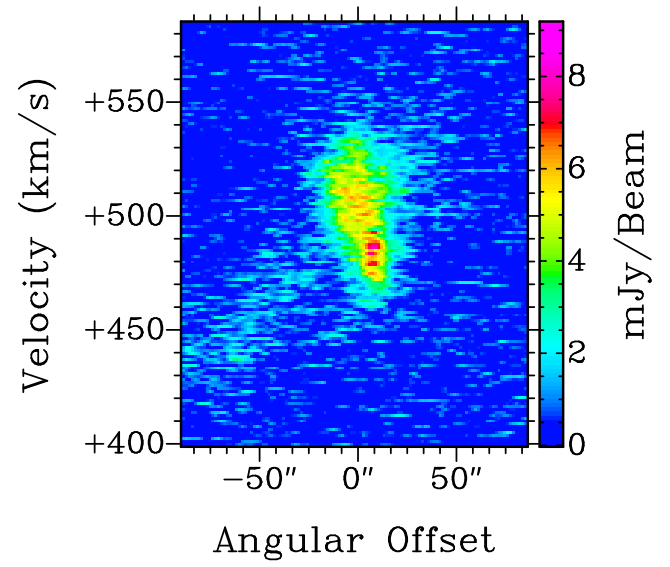

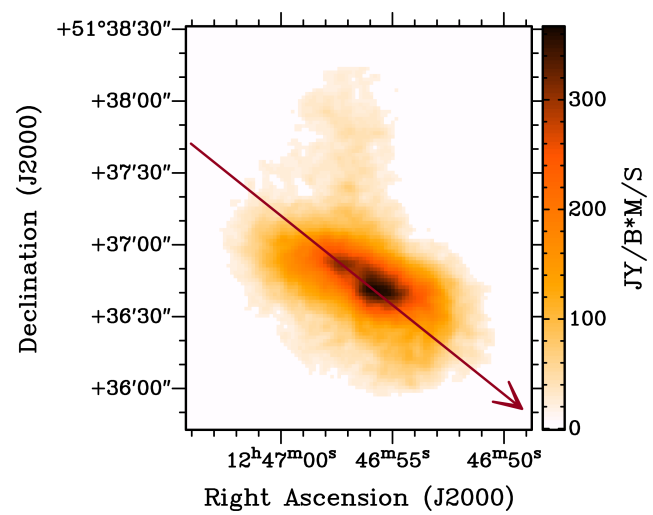

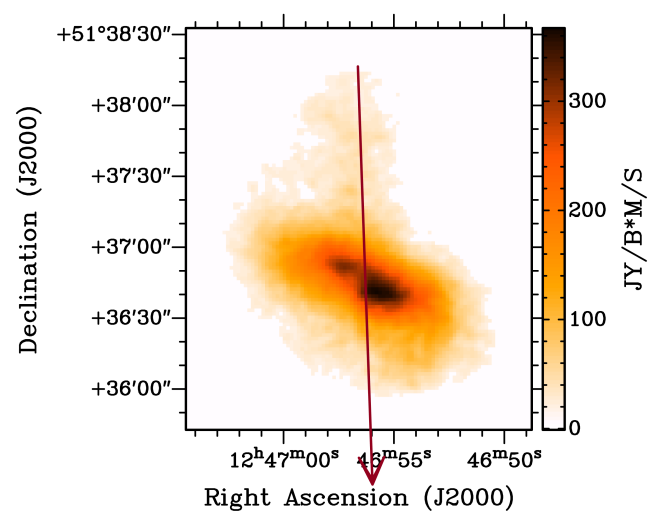

Fig. 10.- Haro 36: The left column contains the P-V diagrams and the right column contains the natural-weighted integrated $\mathrm{H}$ I map with a red arrow indicating the location of the corresponding slice through the galaxy and pointing in the direction of positive offset. The first row is a slice through both central peaks, the second row corresponds to the optical major axis.

the P-V diagram as faint emission. The faint northern extension emission begins at the far left of the P-V diagram and $\sim 430 \mathrm{~km} \mathrm{~s}^{-1}$ and meets the main body of the galaxy at $\sim 485 \mathrm{~km} \mathrm{~s}^{-1}$ and angular offset of $16^{\prime \prime}$. To see if either of the two central H I peaks was connected in velocity space with the northern extension, two P-V diagrams were made with slices through the northern extension, and the approximate centers of each H I peak; shown in the first and second rows of Figure 11. These P-V diagrams do not reveal any obvious connection in velocity space between either of the two H I peaks and the northern extension. However, there is an interesting feature that appears in the P-V diagram in the 

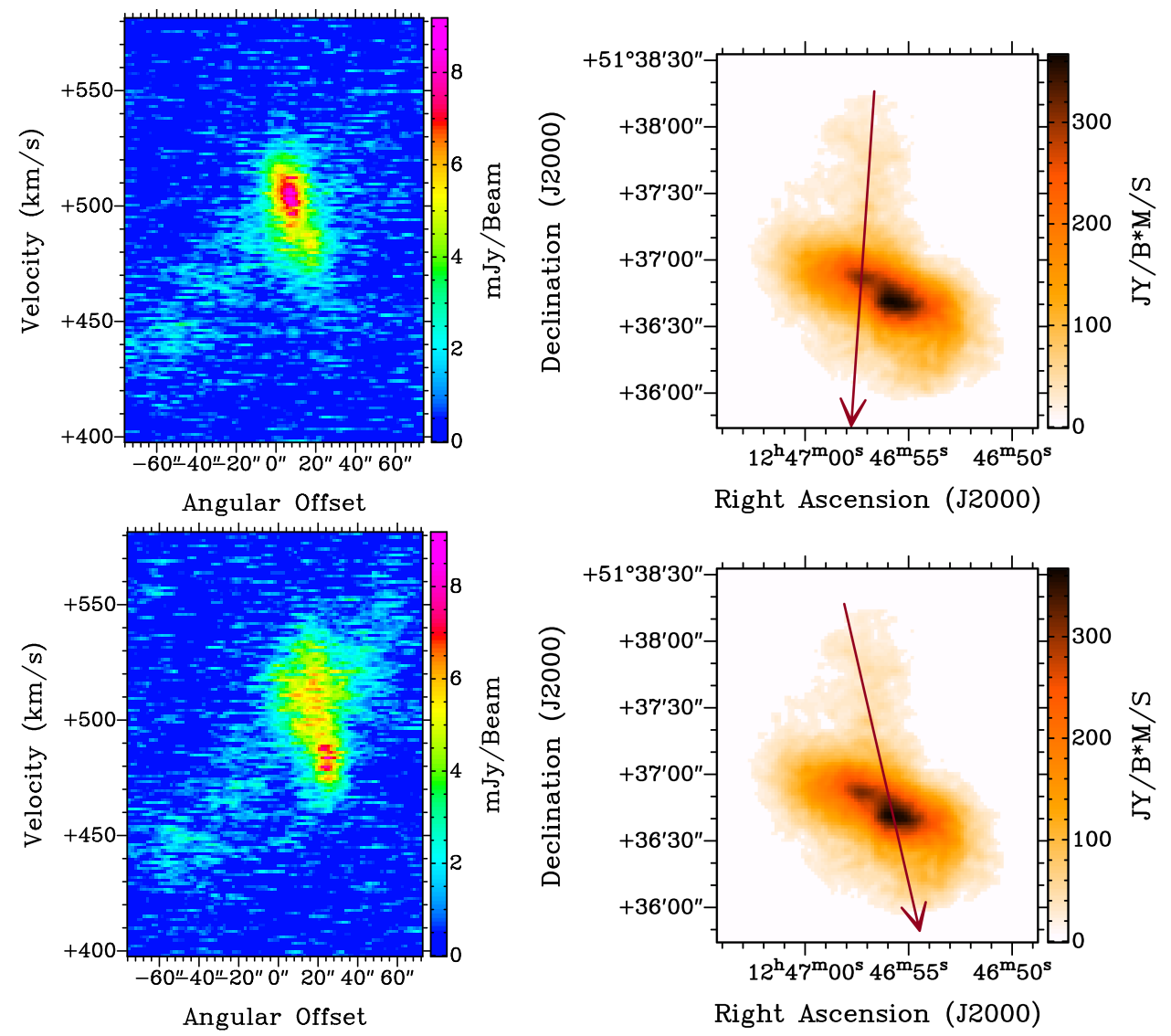

Fig. 11.- Haro 36: The left column contains the P-V diagrams and the right column contains the natural-weighted integrated H I map with a red arrow indicating the location of the corresponding slice through the galaxy and pointing in the direction of positive offset. The first row is a slice through the northern extension and the northeast peak. The second row is a slice through the northern extension and the southwest peak.

bottom row of Figure 11 through the northern extension and southwest peak; the northern extension can be seen starting in the bottom-left region of the P-V diagram and increasing in velocity until it meets the main body of the galaxy, then, on the other side of the main body emission, in the top-right corner of the P-V diagram, the trend of increasing velocity continues from $\sim 530 \mathrm{~km} \mathrm{~s}^{-1}$ at an offset of $40^{\prime \prime}$ to $\sim 560 \mathrm{~km} \mathrm{~s}^{-1}$ and at approximately the same slope that was seen in the emission of the northern extension.

To investigate this possible connection between the northern extension and the southeast region of Haro 36's main body further, I have made four P-V diagrams of 
identical position angle and central declination that change in central RA. These four diagrams and their associated slices are shown in Figure 12; each slice is labeled with a letter a-d. These four slices were chosen because they show the connection between the northern extension and the southeast region most clearly. To explain these graphs clearly, I have included a labeled version of diagram "b" in Figure 13 where the most important features of the P-V diagrams have been labeled. The northern extension is labeled " 3 " and it extends from $\sim 430-485 \mathrm{~km} \mathrm{~s}^{-1}$. There are two features associated with the southeastern tenuous emission that create a looping pattern in the top-right corner of Figure 13: the top of the loop is labeled "1" and covers velocities from $~ 545-570 \mathrm{~km} \mathrm{~s}^{-1}$ and angular offset of $16^{\prime \prime}-50^{\prime \prime}$, while the bottom part of the loop is labeled " 2 " and covers velocities from $\sim 525-570 \mathrm{~km} \mathrm{~s}^{-1}$ and angular offset of $42^{\prime \prime}-50^{\prime \prime}$. I will use these labels throughout the rest of the dissertation.

Beginning with diagram "a" the northern extension (Region 3) appears in the left side of the P-V diagram and Region 2 appears in the top-right of the P-V diagram. Region 1 is not visible in this diagram. Then, in diagram "b," as RA decreases, something interesting occurs in the top-right of the P-V diagram; Region 1 begins to appear. It is also interesting to note that in diagram "b," the emission from the main body of Haro 36 appears distorted between the velocities $\sim 490-510 \mathrm{~km} \mathrm{~s}^{-1}$, where the yellow and red regions of the main body emission appear to connect Region 3 and Region 2. In diagram "c" the emission in Region 1 becomes stronger. Then, in diagram "d", Region 2 becomes fainter than in previous P-V diagrams and the emission in Region 1 appears stronger. It is likely that that Regions 1, 2, and 3 form a tidal tail that is in the foreground of the main body of Haro 36, distorting the apparent velocity field of the main body.

Region 1 is associated with the tenuous emission to the southwest of the main body in Haro 36 and is strongest at lower RAs (positions further west). Region 2, which is also associated with the tenuous emission to the southwest of the main body, is strongest at 

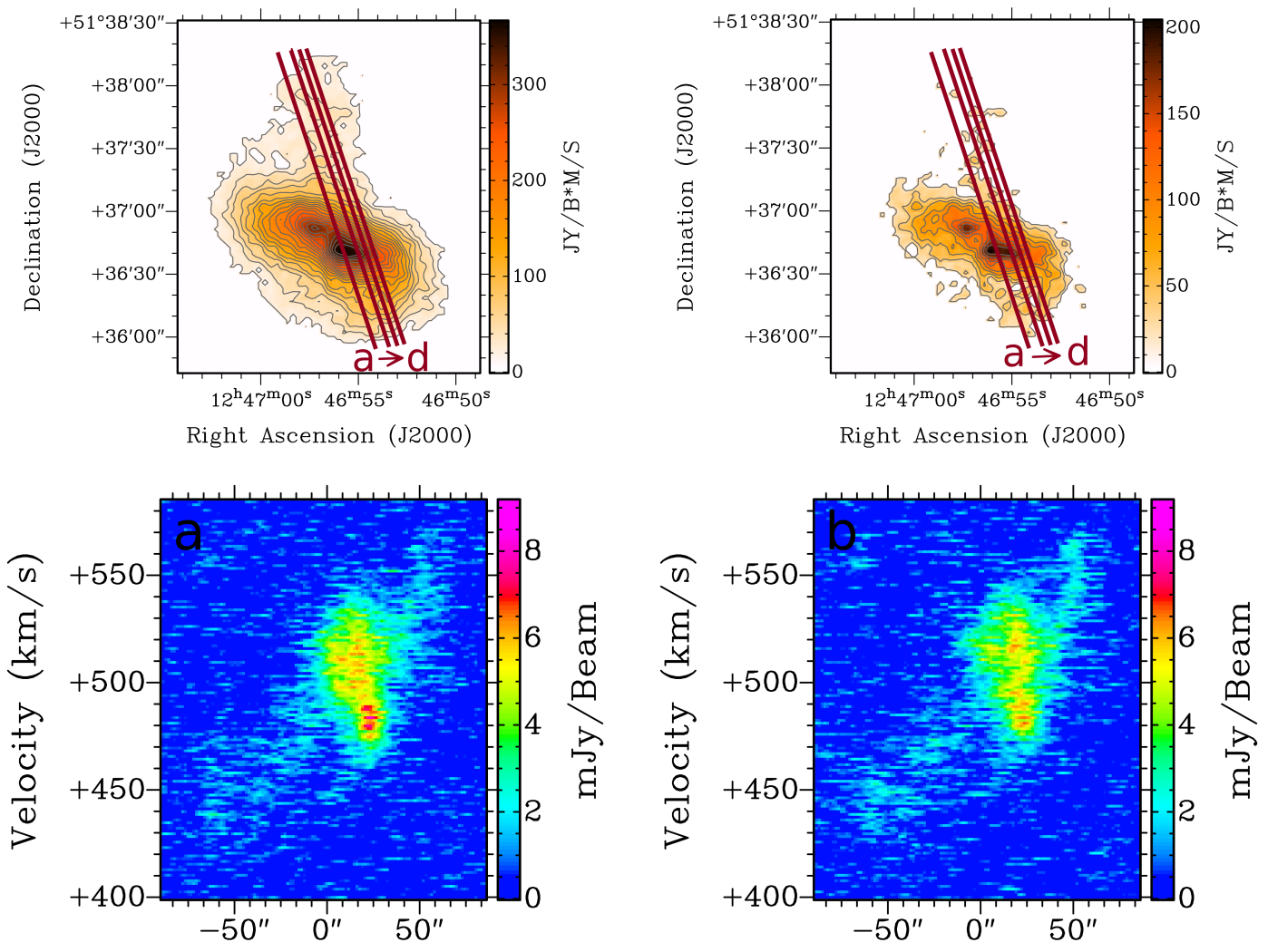

Angular Offset
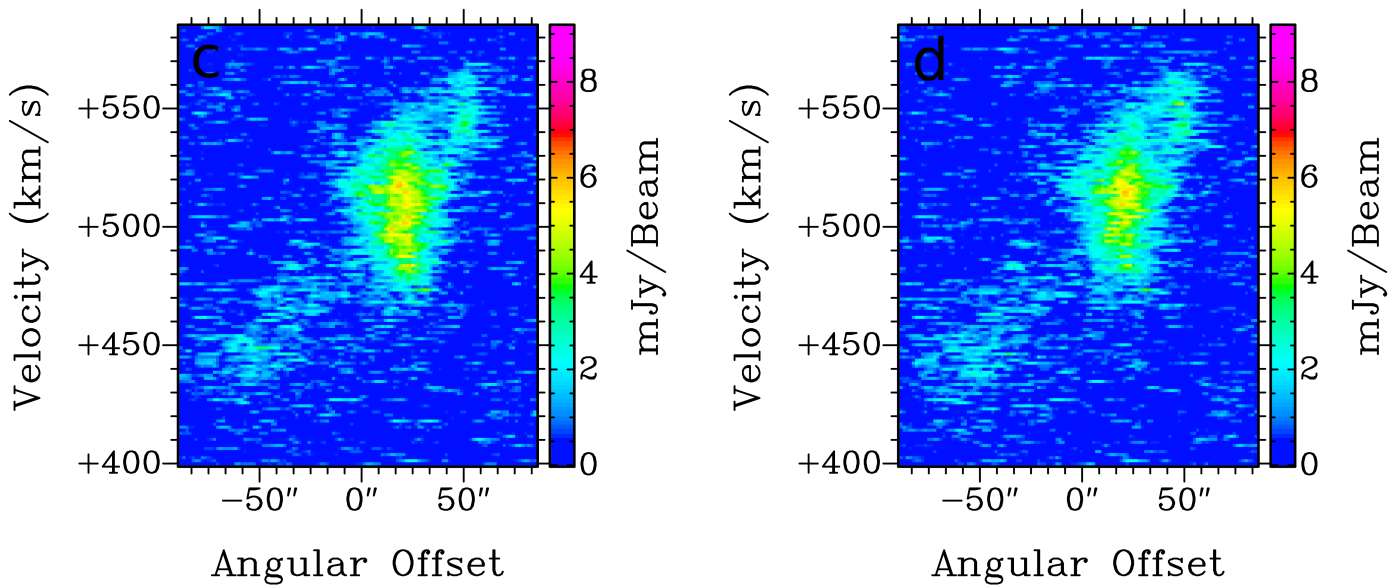

Fig. 12.- Haro 36: The top row contains the natural-weighted (left) and robust-weighted (right) integrated $\mathrm{H}$ I maps with red lines indicating the approximate locations of the slices through the galaxy used in the P-V diagrams (a-d) located below.

higher RAs. The P-V diagrams of Regions 1 and 2 show that they have slightly different velocities over the same positions. At an angular offset of $\sim 50^{\prime \prime}$ they both have a velocity 


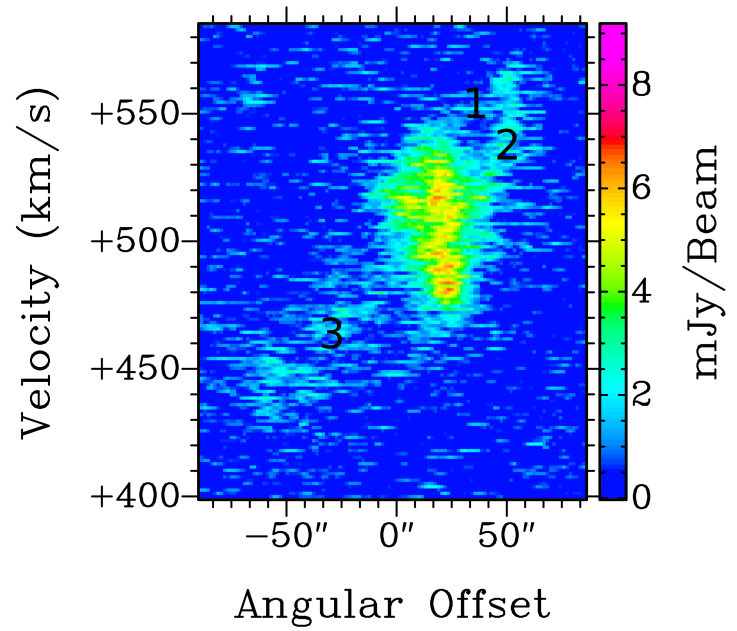

Fig. 13. - Haro 36: A labeled version of P-V diagram b from Figure 12.

of $\sim 565 \mathrm{~km} \mathrm{~s}^{-1}$. This overlap in velocity and position suggests that they could be part of the same structure. With Region 3 apparently having the same slope in the P-V diagram, these three regions, if physically connected, appear to create a structure that (with respect to the systemic velocity of the galaxy; $502 \mathrm{~km} \mathrm{~s}^{-1}$ ) is blueshifted in the north, and redshifted in the southwest. The main body of the galaxy is blueshifted in the northeast end of the disk and redshifted in the southwest of the disk. The tenuous structure formed by Regions 1,2, and 3 is thus following the same general kinematic trends as the main body, but covering a larger velocity range. Therefore, together the three regions may form a gas structure that has been pulled from the outer regions of the main disk to a higher velocity range, also known as a tidal tail.

In P-V diagram "b" from Figure 12, the yellow and red emission in the main body appear to continue the slope in Region 3, connecting it to Region 2 . This could be explained by the tidal tail being in the foreground of the galaxy and therefore disturbing the velocities of the main body in the P-V diagram "b." This connecting slope in Figure 12(b) also suggests that what we can see of the tidal tail begins in Region 1, which increases in 
velocity until it reaches its maximum angular offset and velocity from the disk, turns into Region 2, which decreases in velocity, eventually sweeping in front of the main body of the galaxy, and finally ending in Region 3.

The velocity dispersion map in bottom of Figure 7 supports this conclusion. High velocity dispersions (20 $\mathrm{km} \mathrm{s}^{-1}$ and above) across the main body of Haro 36 appear to connect the southwestern, tenuous emission associated with Regions 1 and 2 to the beginning of the northern extension. These high velocity dispersions are likely to be associated with the tidal tail being in the foreground of the main body, distorting the main body's velocity field.

The tidal tail crossing in front of the main body does not, however, appear to explain the kinematically distinct southwest H I peak. Evidence of this can be seen in Figure 12(a); slice "a" goes through the southwest H I peak, but the high H I density emission associated with the southwest $\mathrm{H}$ I peak on the P-V diagram appears in red at a velocity of $\sim 480 \mathrm{~km}$ $\mathrm{s}^{-1}$ and an angular offset of $\sim 25^{\prime \prime}$. That peak in emission in the P-V diagram is lower in velocity by $\sim 20 \mathrm{~km} \mathrm{~s}^{-1}$ from the line that would connect Regions 2 and 3 . Therefore it is not likely that the tidal tail is the reason for the disturbed kinematics seen in the southwest H I peak.

In Haro 36's robust weighted integrated H I intensity map from the VLA shown in Figure 8 , there is a looping structure in the southwest region of the disk, which may be associated with Regions 1 and 2 of the tidal tail. To see this clearly, slices a-d have been overlaid on the robust weighted map in the top-right of Figure 12. In slice "a" Region 2 is visible and not Region 1 , and in the robust column density map, slice "a" crosses the left side of the loop. In slice " $b$ " Region 1 begins to appear and in the robust column density map, slice "b" moves further west. In slices "c" and "d" Region 1 becomes prominent and in the robust column density map slices "c" and "d" cross through the right side of the 
loop. Therefore, the looping structure in the southwest region of the robust column density map could be a physical turnaround in the tidal tail (in the line of sight). Since Region 2 is always present in the P-V diagram, it is likely that tenuous emission from Region 2 crosses in front of the emission from Region 1 in the line of sight.

The V-band image of Haro 36 also shows possible signs of the tidal tail. Stellar features in the V-band that may be associated with Haro 36's tidal tail are enclosed in red circles in Figure 14. The two bottom circles indicate stellar features with sharp edges that appear to line up well with the looping structure seen in the robust maps (see the bottom map in Figure 8). These sharp stellar features could indicate a stellar component to the tidal tail. The top circle also appears to be a morphologically pointed feature and lines up well with the beginning of the $\mathrm{H}$ I northern extension. A tidal tail in the north-south direction would also explain the north-south elongation in the outer isophotes in the V-band map.

Haro 36's isolation with respect to other galaxies, apparent lack of connection to NGC 4707 (as seen in the GBT data in Figure 9) and likely tidal tail appear to indicate that Haro 36 may be a dwarf-dwarf merger. 


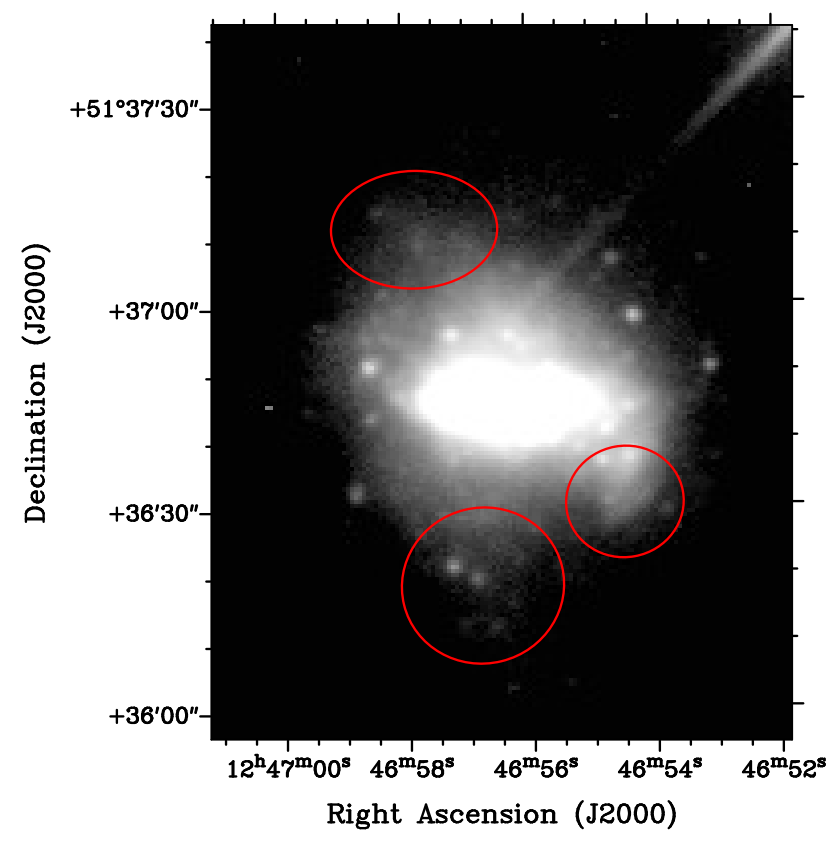

Fig. 14.- Haro 36: The V-band emission seen in Figure 6; the red circles indicate the location of features that may be a result of the tidal tail in Haro 36. 
8. Results: Mrk 178

\subsection{Stellar Component}
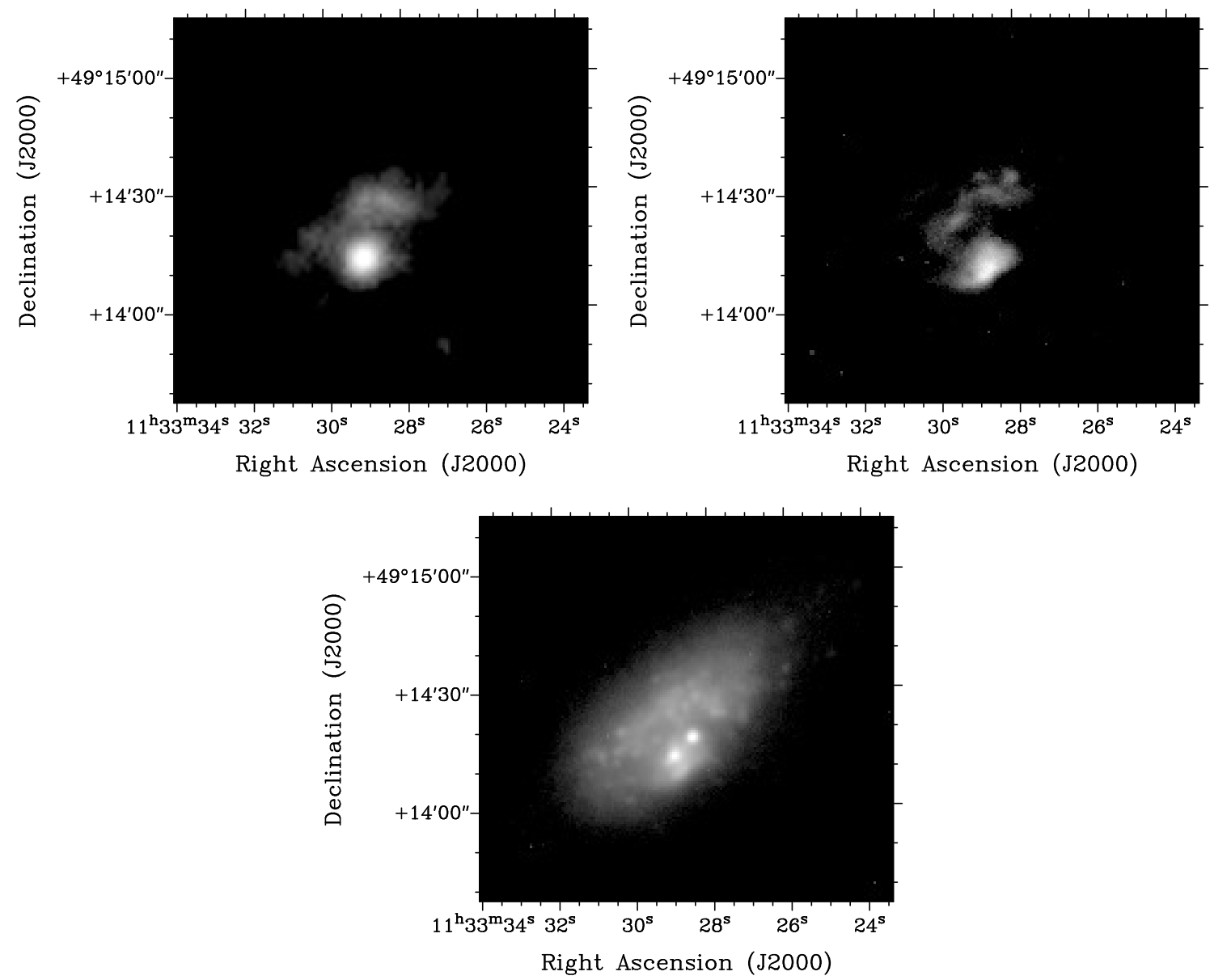

Fig. 15.—Mrk 178 Left: FUV.; Right: H $\alpha . ;$ Bottom: V-band.

The FUV, $\mathrm{H} \alpha$, and V-band maps of Mrk 178 are shown in Figure 15. The FUV data were taken with GALEX, and the $\mathrm{H} \alpha$ and V-band data were taken with the Lowell Observatory $1.8 \mathrm{~m}$ Perkins Telescope. The $\mathrm{H} \alpha$ traces star formation within the last $10 \mathrm{Myr}$ (Hunter \& Elmegreen 2006). All three of these maps share a common feature in their morphology; there appears to be a region of high stellar density to the south, then there is a curved structure of stars, north of the high stellar density region, that curves to the west. 

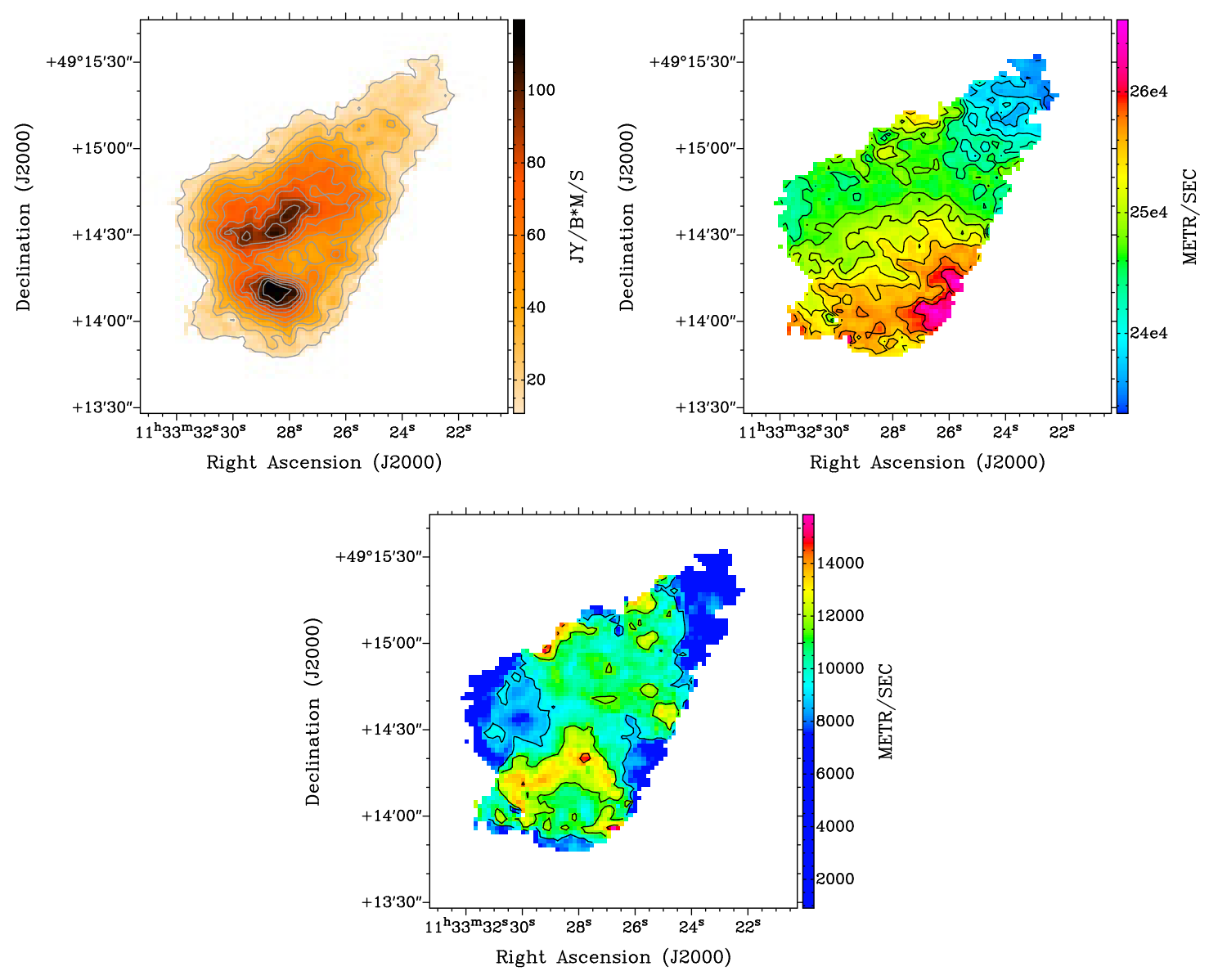

Fig. 16.- Mrk 178's natural-weighted moment maps. Upper left: Integrated H I intensity map; contour levels are $1 \sigma \times(2,4,6,8,10,12,14,16,18,20)$ where $1 \sigma=$ $6.68 \times 10^{19}$ atoms $\mathrm{cm}^{-2}$. Upper right: Intensity weighted velocity field; contour levels are $235 \mathrm{~km} \mathrm{~s}^{-1}$ to $260 \mathrm{~km} \mathrm{~s}^{-1}$ separated by $2.5 \mathrm{~km} \mathrm{~s}^{-1}$. Bottom: Velocity dispersion field; contour levels are $9,11.5,14 \mathrm{~km} \mathrm{~s}^{-1}$.

\subsection{VLA H I Morphology}

The natural weighted integrated H I intensity map as measured by the VLA is shown in Figure 16. There are two distinct regions of high density; one to the north and one to the south. The dense region to the north has three internal peaks, while the region to the south has one internal peak. These two high density regions appear to be part of a ring-like structure that was also seen in Stil \& Israel (2002). There is also tenuous H I to the northwest that creates an overall tadpole-like morphology in the map. 

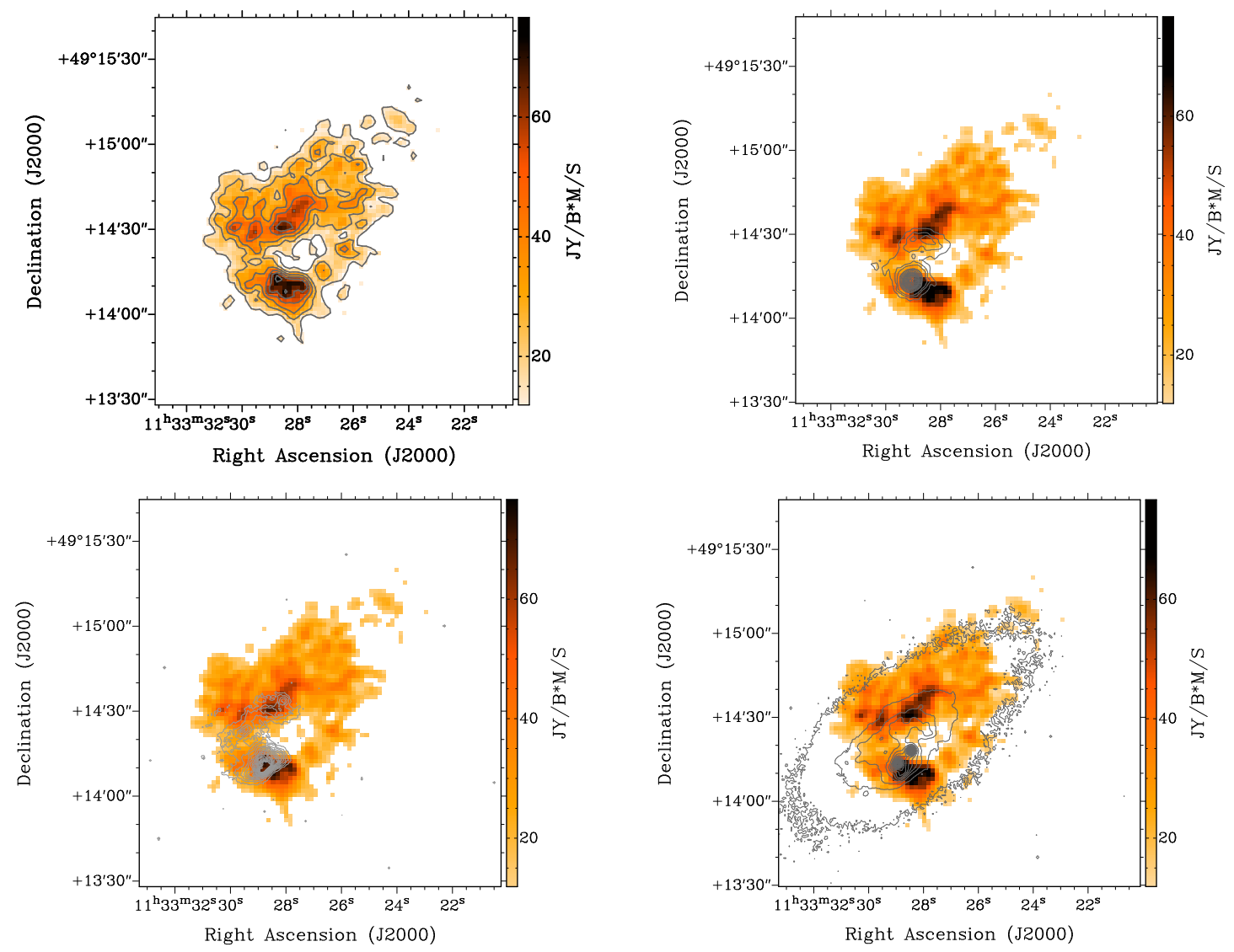

Fig. 17.- Mrk 178's robust-weighted moment maps. Upper left: Integrated H I intensity map; contour levels are $1 \sigma \times(2,4,6,8,10,12)$ where $1 \sigma=1.95 \times 10^{20}$ atoms cm $^{-2}$. Upper right: Integrated H I intensity map colorscale and FUV contours. Bottom left: Integrated $\mathrm{H}$ I intensity map colorscale and $\mathrm{H} \alpha$ contours. Bottom right: Integrated $\mathrm{H}$ I intensity map colorscale and V-band contours.

The integrated H I intensity map of the robust weighted data is shown in the top-left of Figure 17. This map shows the broken ring-like structure at a higher resolution. Plots of the FUV, $\mathrm{H} \alpha$, and V-band contours over the colorscale of the robust weighted integrated $\mathrm{H}$ I intensity map are also shown in Figure 17. Here we see that the curved feature in the stellar components follows the morphology of the broken ring-like structure in the $\mathrm{H} I$ and that the region of high stellar concentration occurs closer to the southern region of high density. 


\subsection{VLA H I Velocity and Velocity Dispersion Field}

The VLA H I velocity field for Mrk 178 can be seen in the top-right of Figure 16. If the tenuous gas creating an extension to the northwest is not included, the velocity field of Mrk 178 is reminiscent of solid body rotation with a kinematic major axis with a PA of roughly $30^{\circ}$ (estimated by eye). The tenuous extension to the northwest has isovelocity contours that are nearly perpendicular to the isovelocity contours of the rest of the H I with a kinematic major axis with a PA of roughly $130-135^{\circ}$ (estimated by eye). The northwest extension has a length of $\sim 920 \mathrm{pc}$. The length of the extension was taken from the natural weighted map from the tip of the northwest edge of the $2 \sigma$ contour to the southeast tip of the $245 \mathrm{~km} \mathrm{~s}^{-1}$ contour. The $245 \mathrm{~km} \mathrm{~s}^{-1}$ contour was chosen as a cutoff for the length because it is the most southeastern isovelocity contour with kinematic characteristics that appear related to those seen in the northwest extension, in that it is nearly perpendicular to the isovelocity contours in the main body.

The VLA H I velocity dispersion field for Mrk 178 is shown in the bottom of Figure 16. The velocity dispersions in Mrk 178 go up to $\sim 16 \mathrm{~km} \mathrm{~s}^{-1}$ but only in a few, small spots. A larger region of the velocity dispersion map in the southeast contains velocity dispersions of $\sim 13 \mathrm{~km} \mathrm{~s}^{-1}$.

\subsection{GBT H I Morphology and Velocity Field}

The integrated H I intensity map as measured with the GBT is shown in the top-left side of Figure 18. The morphology of the GBT H I is very similar to that of the VLA H I morphology. This can be seen in the map at the bottom of Figure 18, where the $5 \sigma$ contour of the GBT data are shown in black, and the $2 \sigma$ contour from the natural weighted VLA column density map is plotted in red. Both extend in the same direction. The GBT data 
are in galactic coordinates, as opposed to RA and Dec, which is why the orientation of the GBT data appear different from that seen in the VLA maps. The rest of the emission in the GBT map is likely to be from noise. The velocity field of the GBT H I data are shown in the top-right side of Figure 18 . There is no obvious velocity gradient in the GBT H I map, however, it may be that the same velocity trends seen in the VLA velocity field are seen again here, where the extension has a velocity field with isovelocity contours perpendicular to those seen in the main body of Mrk 178.

\subsection{H I Mass}

Mrk 178's total H I mass from the VLA is $8.7 \times 10^{6} M_{\odot}$, and from the GBT is $1.1 \times 10^{7} M_{\odot}$. The VLA recovered $79 \%$ of the GBT mass. The mass of the northwest extension in the VLA natural weighted map was also calculated using BLSUM. The border which separates the northwest extension from the rest of the H I in Mrk 178 was again defined by the isovelocity contour of $245 \mathrm{~km} \mathrm{~s}^{-1}$ that is furthest northwest in the map. The mass of the northwest extension is $7.5 \times 10^{5} M_{\odot}$ or $8.6 \%$ of the total VLA H I mass.

\section{Discussion: Mrk 178}

Previous claims of Mrk 178 being a merger (Pustilnik et al. 2001) appear morphologically possible if the two dense regions of $\mathrm{H}$ I are the remnants of two merging dwarfs. If they are the cores of two galaxies in the process of merging, then the gas would likely be quite disturbed with velocity dispersions $20 \mathrm{~km} \mathrm{~s}^{-1}$ and above. However, the $\mathrm{H} \mathrm{I}$ velocity dispersions in Mrk 178 are quite low; most of the gas has velocity dispersions of $\sim 10-13 \mathrm{~km} \mathrm{~s}^{-1}$, indicating that Mrk 178 is not likely two currently merging galaxies. A 

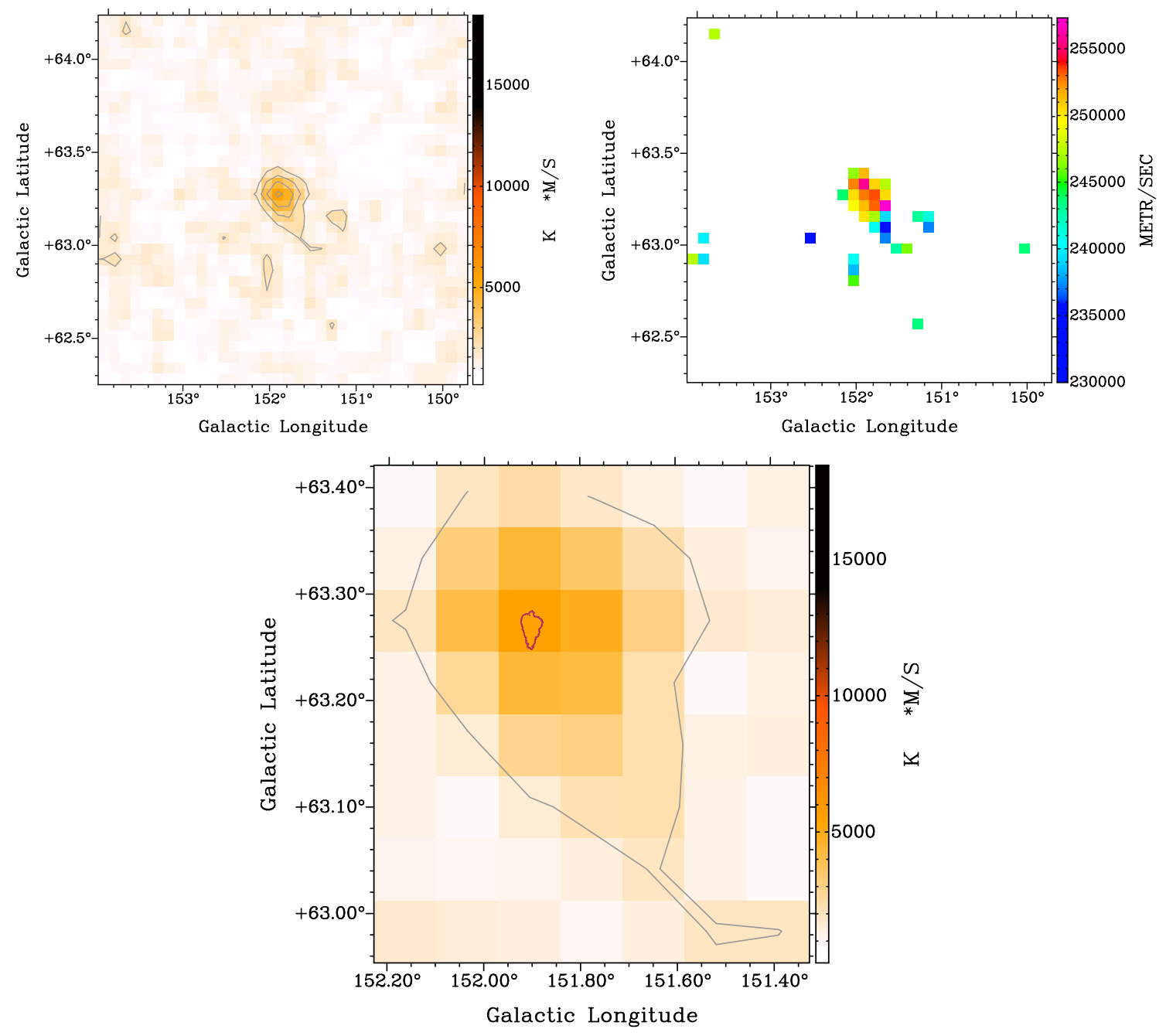

Fig. 18.- Mrk 178's GBT moment maps. Upper left: Integrated H I intensity map; contour levels are $1 \sigma \times(5,8,11,14,17,20,23,26,29,32,35,38,41,44,47)$ where $1 \sigma=2.89 \times 10^{17}$ atoms $\mathrm{cm}^{-2}$. Upper right: Intensity weighted velocity field Bottom: A comparison between Mrk 178's GBT data and natural weighted VLA data; the GBT data are the colorscale and grey contour of $1.5 \times 10^{18}$ atoms $\mathrm{cm}^{-2}$, and the VLA data are outlined by the red contour of $1.3 \times 10^{20}$ atoms cm $^{-2}$.

more likely explanation for the H I morphology of Mrk 178 is a hole-shell structure that has been formed by stellar winds and supernovae.

Holes can be identified using P-V diagrams; the emission around a hole will create an empty ring or partial ring appearance in the $\mathrm{P}-\mathrm{V}$ diagram when a hole is present. The ring appearance in $\mathrm{P}-\mathrm{V}$ diagrams occurs because the gas is moving like an expanding bubble; if 
the systemic velocity of the bubble's center is $0 \mathrm{~km} \mathrm{~s}^{-1}$, then the front of the bubble will be expanding toward the observer and the back of the bubble will be expanding away from the observer, while the edge ${ }^{16}$ of the bubble will expand into the plane of the projected map with no Doppler shift. So, when a slice for a P-V diagram is taken through an expanding hole, the slice will first go through an edge of the hole with no Doppler shift. Next, it will go through the front and the back of the projected hole at the same time, resulting in emission on the P-V diagram at two different, separate velocity ranges with little to no emission between them ${ }^{17}$. Finally, the slice will go through the other edge of the hole with no Doppler shift. On a P-V diagram this will create a ring-like shape. However, holes can break through one or both sides of the disk, therefore it is possible to be missing one or more sides of the ring on the $\mathrm{P}-\mathrm{V}$ diagram.

In the top row of Figure 19, a hole is visible in Mrk 178's P-V diagram of a slice taken between the two dense $\mathrm{H}$ I regions. The hole creates a partial ring defined by the offsets of $-4^{\prime \prime}$ and $12^{\prime \prime}$, and velocities of $\sim 250 \mathrm{~km} \mathrm{~s}^{-1}$ and $\sim 260 \mathrm{~km} \mathrm{~s}^{-1}$. The higher velocity side of the ring may be tenuous because of the gas on that side of the hole being very tenuous or that side of the hole having blown out of the $\mathrm{H}$ I disk. The P-V diagrams of the two dense $\mathrm{H}$ I regions in the second and third rows of Figure 19 show that they have approximately the same average velocity of $\sim 250-255 \mathrm{~km} \mathrm{~s}^{-1}$ and about the same average velocity as the edges of the hole seen in the P-V diagram in the first row of Figure 19. It is therefore possible that the regions of high $\mathrm{H}$ I column density in the VLA maps are part of a shell that has been created by the hole between them. The robust weighted integrated

\footnotetext{
${ }^{16}$ In the line of sight a bubble will look like a circle to the observer, since the bubble will be seen in projection and the observer will be looking through the most gas at the outer edge of the projected bubble. Therefore edge here refers to the outside edge of the projected circle.

${ }^{17}$ The hole itself will have little neutral gas inside since most of it will have been ionized by stellar radiation or swept out by stellar winds and/or supernovae explosions.
} 

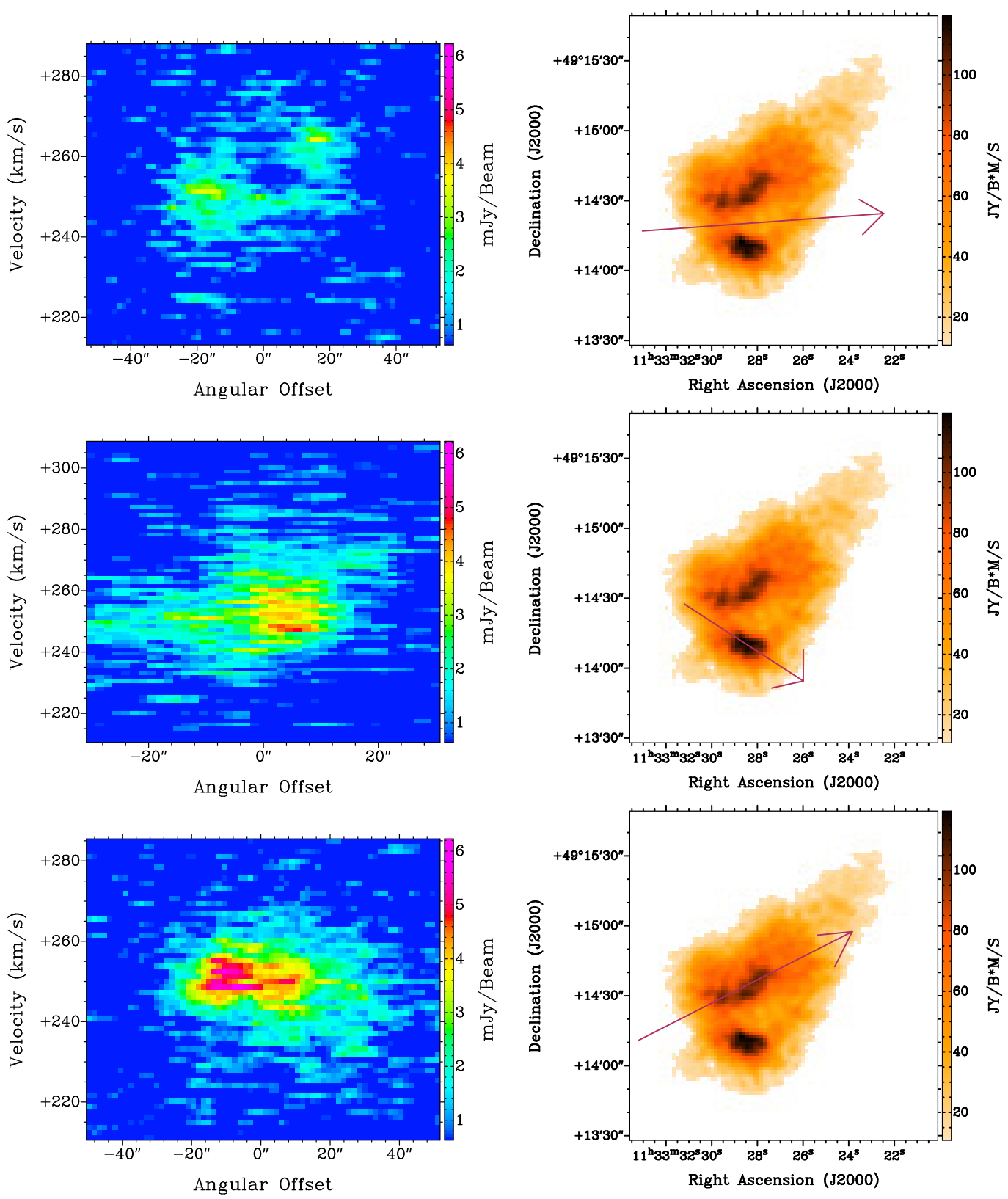

Fig. 19.- Mrk 178: The left column contains the P-V diagrams and the right column contains the natural-weighted integrated $\mathrm{H}$ I map with a red arrow indicating the location of the corresponding slice through the galaxy and pointing in the direction of positive offset. The first row is a slice through the hole in Mrk 178, the second row is a slice through the southern H I peak, and the third row is a slice through the northern H I peak.

H I intensity map as measured by the VLA shows the structure of the potential shell more clearly. 
With the higher velocity side of the shell not clearly defined, it is not possible to get an exact expansion rate for the hole, however the velocity of the dense edge of the shell can be used to get an estimate of the expansion. When one side of the ring is missing it is assumed that the approximate average velocity of the edge of the hole is the approximate velocity of the central region of the hole. The approximate central velocity can then be compared to the with the velocity of the front or back of the hole to get a current expansion velocity. When the expansion rate is estimated for Mrk 178, the velocity of the center of the hole is found to be $\sim 255 \mathrm{~km} \mathrm{~s}^{-1}$, and with the back side of the hole at $\sim 250 \mathrm{~km} \mathrm{~s}^{-1}$, this results in an expansion velocity of $5 \mathrm{~km} \mathrm{~s}^{-1}$. The expansion velocity of the hole in conjunction with the approximate size of the hole (estimated from the P-V diagram in the top row of Figure 19), provides a rough estimate of the age of the hole. With a major axis radius of $\sim 16^{\prime \prime}$ or $\sim 300 \mathrm{pc}$ (at a PA of $\sim 94^{\circ}$ ), the hole, at $5 \mathrm{~km} \mathrm{~s}^{-1}$, would have taken approximately $60 \mathrm{Myr}$ to form. The calculated age of the hole is an upper limit as the expansion rate of the hole may have been much faster when it first formed.

Using models from Chevalier (1974) it is possible to get a rough estimate of the energy needed to create Mrk 178's hole. The energy can be calculated using equation 26 from Chevalier (1974):

$$
E_{0}=5.3 \times 10^{-7} n_{0}^{1.12} v_{s h}^{1.40} R^{3.12}
$$

where $E_{0}$ is the initial energy in units of $10^{50} \mathrm{ergs}, n_{0}$ is the initial volumetric density of the gas in atoms $\mathrm{cm}^{-3}, v_{s h}$ is the velocity of the hole's expansion, and $R$ is the radius of the hole. For Mrk 178's hole, $n_{0}$ was taken to be the approximate density of the surrounding gas. The fourth contour from the bottom of the natural weighted contours was used to calculate $n_{0}$. That contour's column density is $5.34 \times 10^{20} \mathrm{~cm}^{-2}$ and, assuming a scale height of $200 \mathrm{pc}$ (reasonable for the inner region of a dwarf galaxy; see Banerjee et al. 2011), $n_{0}$ is approximately $0.5 \mathrm{~cm}^{-3}$. Using these parameters results in an energy of $\sim 10^{52}$ ergs. 
Using the energy calculated above and models from McCray \& Kafatos (1987) it may be possible to get rough estimates of the number of stars more massive than $10^{7} M_{\odot}$ necessary to create the hole. However, it must be noted that the equations in McCray \& Kafatos (1987) are for holes that have not broken through the disk, which may have occurred with Mrk 178's hole. Combining equations 3 and 6 from McCray \& Kafatos (1987), gives a timescale for the hole to be created in units of $10^{7}$ years $\left(t_{7}\right)$ :

$$
t_{7}=\left(\frac{R}{97}\right)^{5 / 2}\left(\frac{4 \times 10^{49} n_{0}}{E_{0}}\right)^{2} .
$$

With Equation 7 the age of the hole is calculated to be $\sim 8$ Myr. The number of stars $\left(N_{*}\right)$ needed to create the hole can also be calculated using this timescale and equation 6 in McCray \& Kafatos (1987):

$$
N_{*}=\frac{E_{0}}{4.0 \times 10^{49} E_{51} t_{7}}
$$

where $E_{51}$ is the energy emitted by a supernova explosion in units of $10^{51}$ ergs. $E_{51}$ is assumed to be $\sim 1 . N_{*}$ is $\sim 300$ for Mrk 178's hole, which is a large number of massive stars that should be visible in the stellar maps.

The region of high stellar density in the V-band has two peaks (see Figure 15). The northwestern peak of high stellar luminosity in the V-band does look as though it could be in the hole as can be seen in the bottom right of Figure 17 where it appears offset to the east of the hole. Kehrig et al. (2013) finds a WR knot ("knot C") in approximately the same location as this region of high stellar luminosity composed of $\sim 1-2 \mathrm{WR}$ stars. The W-R knot indicates that there has been star formation in the last few Myr at the location of the northwestern stellar peak which may be inside the hole.

The stellar content that is not in the line of sight of the hole is also very interesting. Each frequency in Figure 17 shows a stellar arc that appears to follow the shape of the potential shell around the hole. The V-band, FUV, and H-alpha represent different star 
formation ages: the V-band represents the star formation over the past Gyr, the FUV represents the star formation over the last 100-200 Myr, and the $\mathrm{H} \alpha$ represents star formation over the past $10 \mathrm{Myr}$. It is therefore interesting to note that the V-band has a tightly curled stellar arc with a small radius, while the $\mathrm{H} \alpha$ has the largest radius for its stellar arc and appears to follow the shape of the dense $\mathrm{H}$ I surrounding the hole the best. The FUV stellar arc has nearly the same morphology as the V-band stellar arc. If the dense $\mathrm{H}$ I emission around the hole is a shell, then the stellar arc could be an indication of star formation taking place in the expanding shell. González-Riestra et al. (1988) calculate the ages of the stars in the V-band's bright stellar knot to the southeast to be older than the stellar knot to the northwest, which could indicate that the southeastern knot is forming in the potential shell. However, it is also possible that these stars in the $\mathrm{H} \alpha$ arc and the southeastern stellar knot simply formed later once the dense gas had been used up from the stars in the FUV stellar arc and the northwestern V-band peak.

The northwest extension is not likely gas that has been blown out from the tenuous side of the hole. The side of the hole that is tenuous is the higher velocity side of the shell, yet the northwest extension contains some of the lowest velocities in the galaxy starting at $\sim 245 \mathrm{~km} \mathrm{~s}^{-1}$. Since the lower velocity side of the shell is still intact, the northern extension was not likely created by blowout of the hole.

Another possible explanation for the northwest extension is that it is a tidal feature, however, if that is the case, then with what object has Mrk 178 interacted? The GBT H I data show no companions at the sensitivity level of the maps within the nearby region, making an interaction unlikely. The velocity dispersions are also too low for Mrk 178 to be a merger. The northwest extension may also be the result of intergalactic gas merging with the galaxy from the northwest and fueling the star formation. The mass of the northwest extension in the VLA maps is only $7.5 \times 10^{5} M_{\odot}$ which is a reasonable mass for a intergalactic starless gas cloud (Sancisi et al. 2008). The GBT data also show the same 
general morphology as the VLA data, as seen in Figure 18. However, modeling is required to confirm if such low velocity dispersions could be obtained when a gas cloud is being acquired by a galaxy. The gas cloud may also be in the foreground or background of the galaxy, not actually impacting the disk. A foreground/background cloud is also consistent with the low velocity dispersions in the disk. 
10. Results: VII Zw 403

\subsection{Stellar Component}
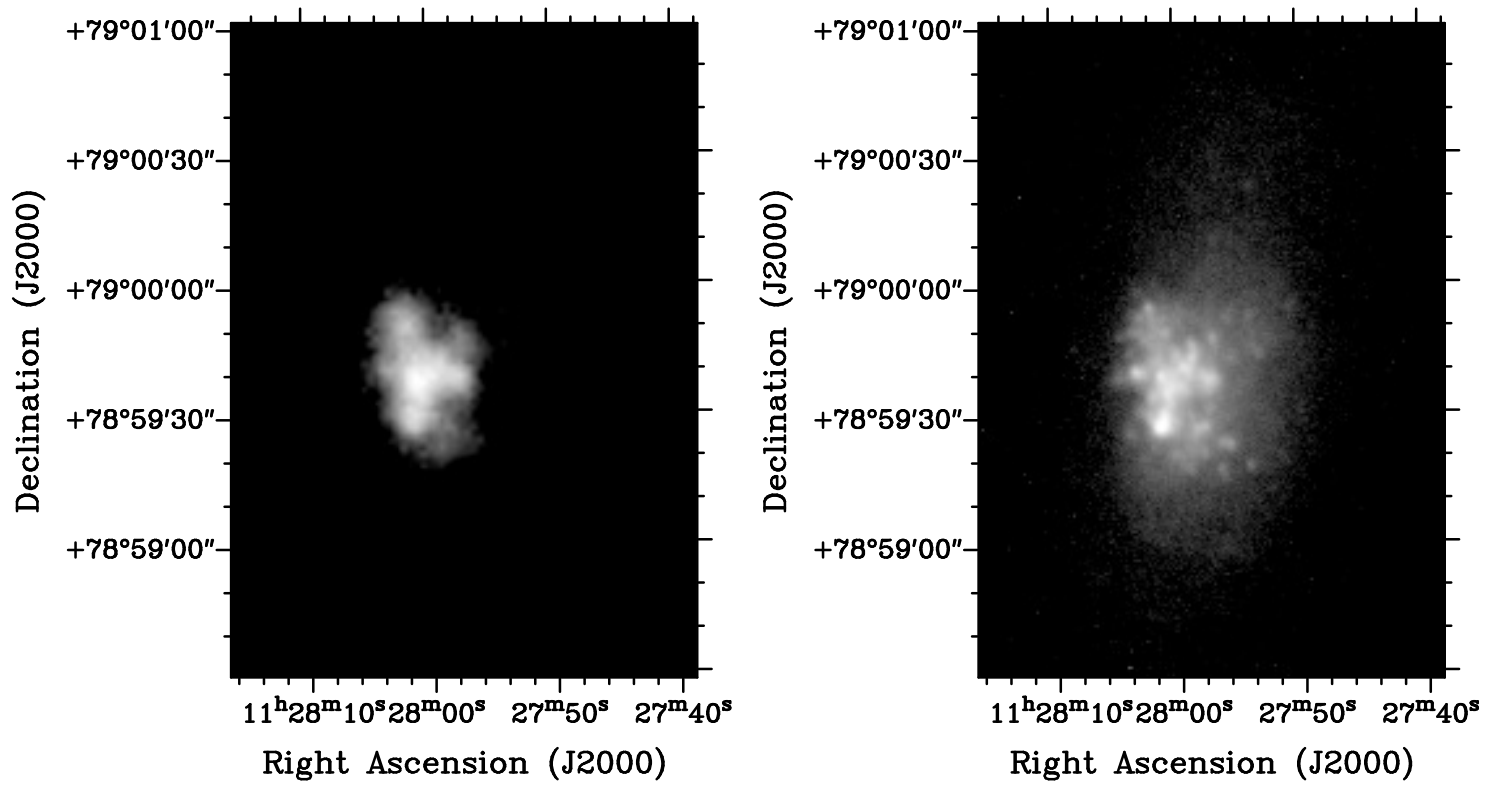

Fig. 20.— VII Zw 403 Left: FUV.; Right: V-band.

VII Zw 403's FUV and V-band data are shown in Figure 20. The FUV data were taken with GALEX and the V-band data were taken with the Lowell Observatory $1.8 \mathrm{~m}$ Perkins Telescope. The FUV morphology is similar to the inner morphology of the V-band.

\subsection{VLA H I Morphology}

VII Zw 403's natural weighted integrated H I intensity map as measured by the VLA is shown in the top-left of Figure 21. The H I emission has a morphological major axis in the north-south direction and is centrally peaked. There is also some detached, tenuous $\mathrm{H}$ I emission just to the south of the main disk that may be associated with VII Zw 403. The robust weighted integrated H I intensity map in the top-left of Figure 22 reveals some structure in the inner region of the $\mathrm{H} \mathrm{I}$; a small piece of emission just north of the densest 
central region that curves toward the east. The FUV and V-band stellar contours are both plotted over the colorscale of VII Zw 403's robust weighted integrated H I intensity map in the top-right and bottom of Figure 22, respectively. The highest isophotes from the FUV and V-band data are located on the highest $\mathrm{H}$ I column density in projection and extend just north of that.

\subsection{VLA H I Velocity and Velocity Dispersion Field}

The VLA velocity field of VII Zw 403 is shown in the top-right of Figure 21. The kinematics of the east side of the galaxy resemble solid body rotation. The kinematics of the west side of the galaxy are generally disturbed with some possible organized rotation in the south. The velocity dispersion field is shown in the bottom of Figure 21 . The dispersions reach near $17 \mathrm{~km} \mathrm{~s}^{-1}$ with the highest dispersions being centrally located.

\subsection{GBT H I Morphology and Velocity Field}

The integrated H I intensity map as measured with the GBT of VII Zw 403 is shown in the left side of Figure 23. The tenuous emission surrounding VII Zw 403 is from the Milky Way. VII Zw 403's velocity range overlaps partially with the velocity range of the Milky Way. These GBT maps were integrated to allow some of that overlap to appear in order to search for any extended emission or companions nearby VII Zw 403. Yet, no matter which velocity range was used in the integration of the data cube, no companions or extended emission from VII Zw 403 were found before confusion ${ }^{18}$ with the Milky Way emission became a problem. Therefore, VII Zw 403 does not appear to have any extra

\footnotetext{
18“Confusion” here refers to VII Zw 403 emission becoming indistinguishable from the Milky Way emission.
} 

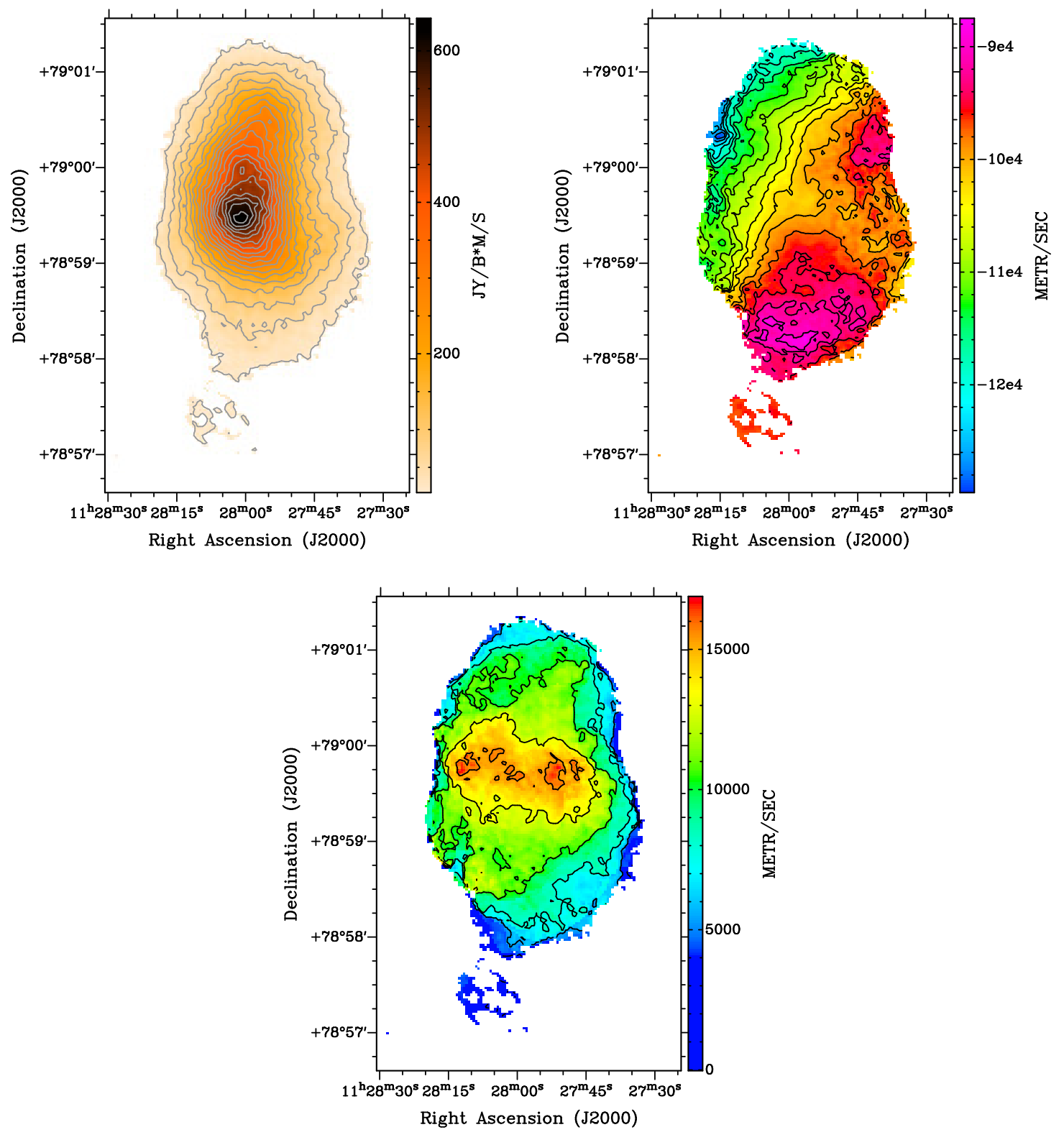

Fig. 21. - VII Zw 403's natural-weighted moment maps. Upper left: Integrated H I intensity map; contour levels are $1 \sigma \times(2,6,10,14,18,22,26,30,34,38,42,46,50,54,58$, $62,66,70)$ where $1 \sigma=3.14 \times 10^{19}$ atoms cm$~^{-2}$. Upper right: Intensity weighted velocity field; contour levels are $90 \mathrm{~km} \mathrm{~s}^{-1}$ to $130 \mathrm{~km} \mathrm{~s}^{-1}$ separated by $2.5 \mathrm{~km} \mathrm{~s}^{-1}$. Bottom: Velocity dispersion field; contour levels are $3 \mathrm{~km} \mathrm{~s}^{-1}$ to $15.5 \mathrm{~km} \mathrm{~s}^{-1}$ separated by $2.5 \mathrm{~km} \mathrm{~s}^{-1}$.

emission or companions nearby at the sensitivity of this map. The GBT H I velocity field is shown in the right side of Figure 23. Here there is no discernible velocity gradient. 

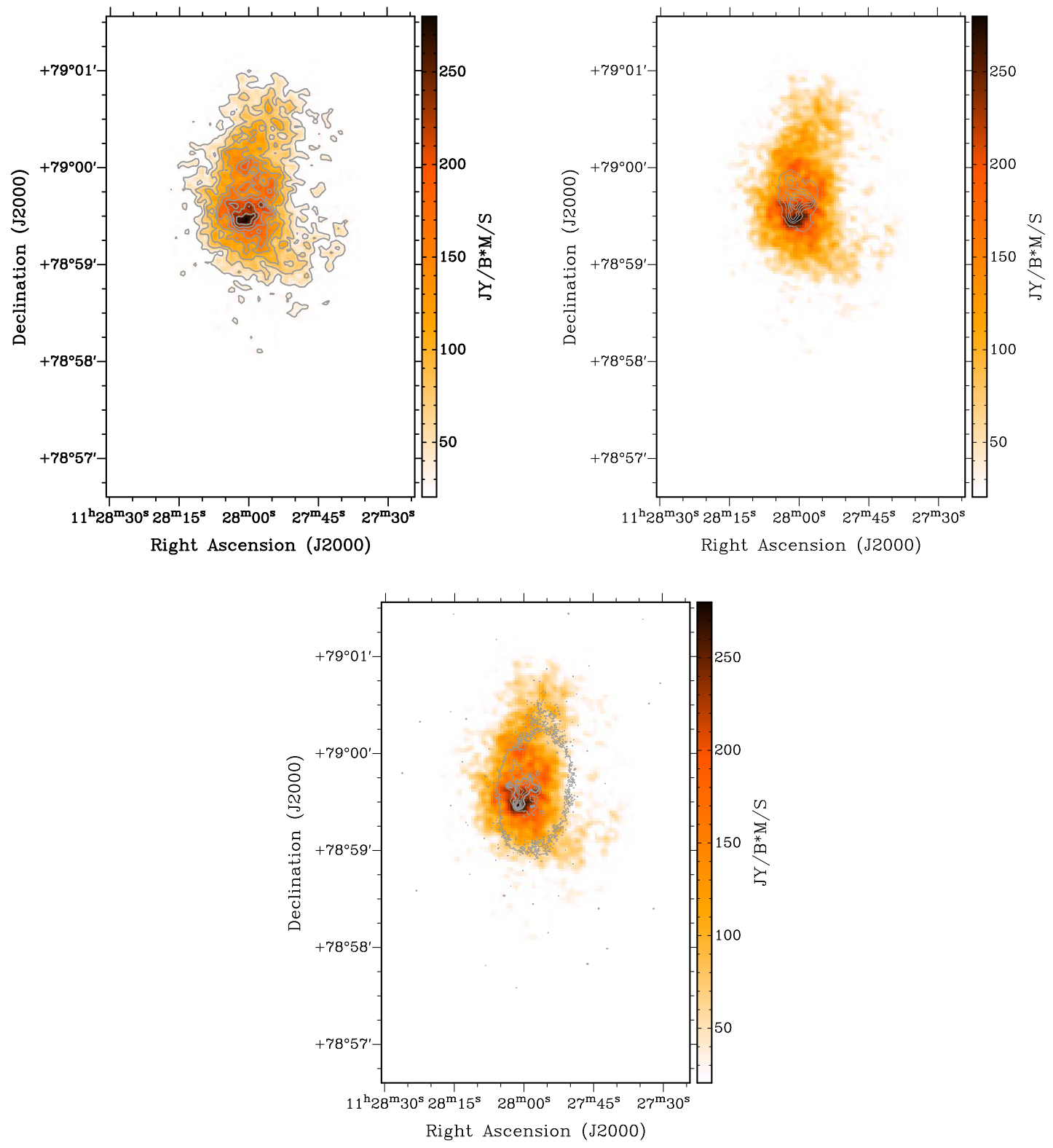

Fig. 22.- VII Zw 403's robust-weighted moment maps. Upper left: Integrated H I intensity map; contour levels are $1 \sigma \times(2,6,10,14,18,22,26)$ where $1 \sigma=1.59 \times 10^{20}$ atoms cm$^{-2}$. Upper right: Integrated H I intensity map colorscale and FUV contours. Bottom: Integrated H I intensity map colorscale and V-band contours.

\subsection{H I Mass}

The total H I mass detected in the VLA natural weighted data is $4.2 \times 10^{7} M_{\odot}$, while the mass from the GBT data is $5.1 \times 10^{7} M_{\odot}$. The Milky Way emission may be contributing 

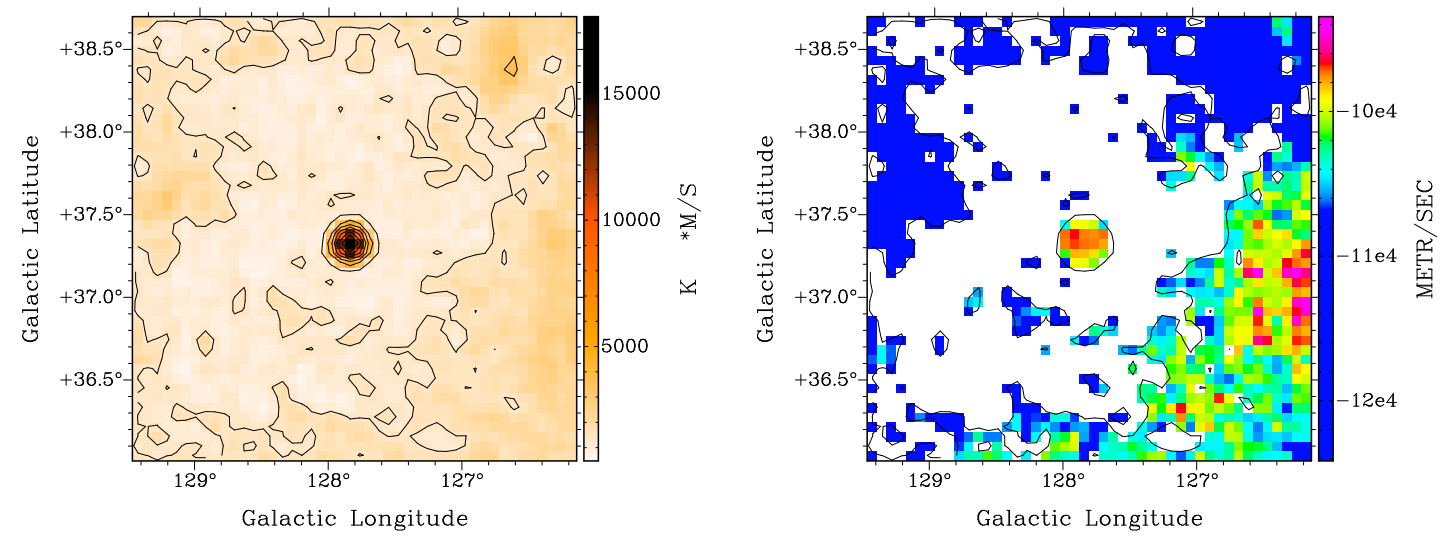

Fig. 23.- VII Zw 403 Left: GBT H I column density; contour levels are $1 \sigma \times(7,17,27$, $37,47,57,67,77,87)$ where $1 \sigma=1.59 \times 10^{17}$ atoms $\mathrm{cm}^{-2}$. Right: GBT H I velocity field.

to some of the mass measured from the GBT data, although it is suspected that the amount is small; the velocity range used in ISPEC was the same as that used to make the integrated $\mathrm{H}$ I intensity map and the box size in which the flux was summed tightly enclosed the VII $\mathrm{Zw} 403$ emission. The VLA was able to recover $82 \%$ of the GBT mass, although the GBT mass could be higher if some emission was missed due to confusion with the Milky Way in some velocity channels, making them unusable for mass calculations.

\section{Discussion: VII Zw 403}

The most noticeable morphological peculiarity in VII Zw 403's VLA data is the detached gas cloud to the south of the disk in the natural weighted integrated $\mathrm{H}$ I intensity map as measured by the VLA. It is unclear if this cloud to the south of the disk is real because it appears at such a low signal-to-noise and could be simply noise. The data are convolved with a $25^{\prime \prime} \times 25^{\prime \prime}$ beam to increase signal-noise, the resulting integrated $\mathrm{H}$ I intensity map is shown in Figure 24. In this map, the southern detached cloud is larger and still there, indicating that it is not likely noise. There is some overlap in the VLA data cube 


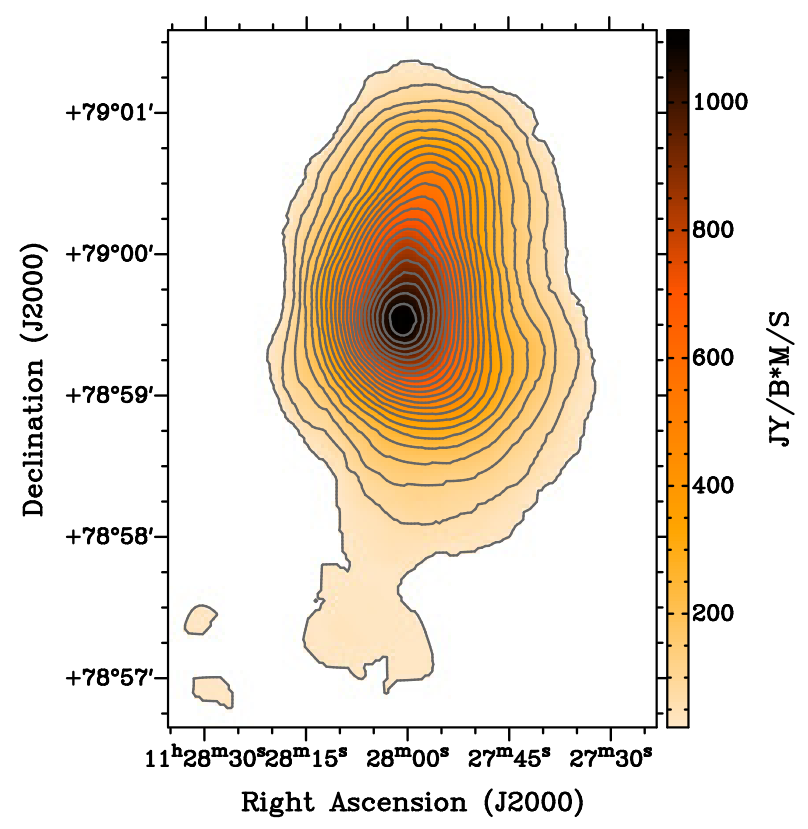

Fig. 24.- VII Zw 403's $25^{\prime \prime} \times 25^{\prime \prime}$ convolved map.

of VII Zw 403's and the Milky Way's velocity range as shown in Figure 25 where VII $\mathrm{Zw} 403$ is in the center of the map and the large clouds surrounding it are from the Milky Way. To make sure that the southern detached cloud is not Milky Way emission in the foreground of VII Zw 403, the channels that contained the southern detached cloud were checked for Milky Way emission. These channels are shown in Figure 26. None of the channels contain obvious Milky Way emission. It is therefore unlikely that the southern detached cloud is a Milky Way cloud in the foreground of VII Zw 403.

Kinematically, VII Zw 403 has two main components: the east side and the west side. The east side of the galaxy has rotation that resembles solid body rotation, while the west side of the galaxy appears to have its velocity field broken into two parts by something in the center declinations. This break in the isovelocity contours is very directional from northeast to southwest. Strikingly, the velocity dispersion map for the natural weighted data also has higher velocity dispersions lining up with the central break in the east side's velocity field. The alignment of the two features can be seen in Figure 27, where the 


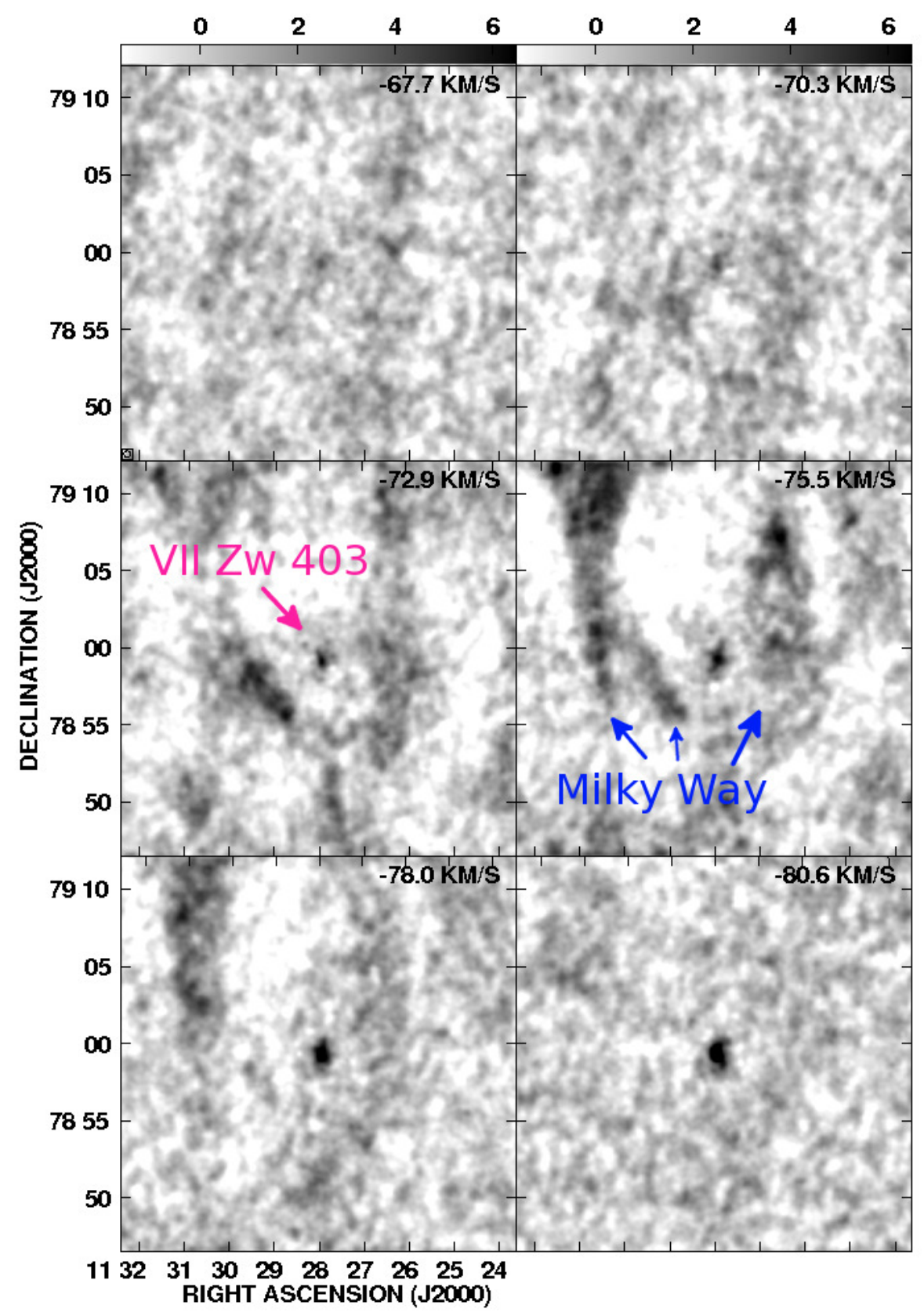

Fig. 25.- VII Zw 403's channel maps from the natural weighted VLA data cube where some overlap of VII Zw 403's and the Milky Way's velocity range occurs.

velocity dispersion field contours have been plotted over the colorscale of the velocity field. 


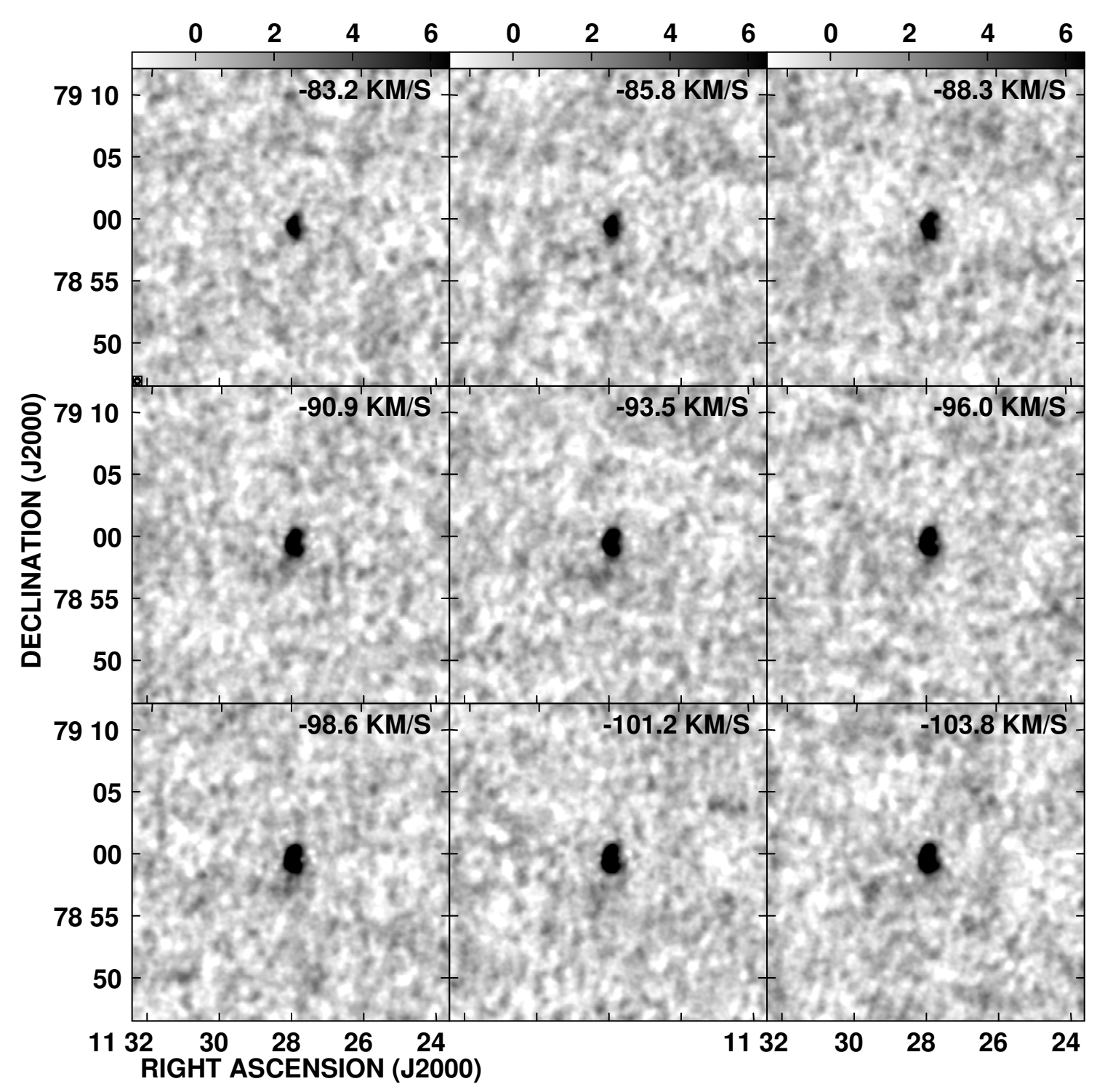

Fig. 26. - The channel maps from the natural weighted VLA data cube from which the extra cloud emission south of VII Zw 403's main body comes.

A P-V diagram through the disturbance using the natural weighted cube is shown in the second row of Figure 28. In this P-V diagram there is a density enhancement from $25^{\prime \prime}$ to $58^{\prime \prime}$ and -110 to $-100 \mathrm{~km} \mathrm{~s}^{-1}$. The rest of the tenuous gas at this angular offset range appears to be at lower negative velocities than $-100 \mathrm{~km} \mathrm{~s}^{-1}$, consistent with the rest of the gas on the west side rotating in a solid body fashion with the east side of the disk. It 

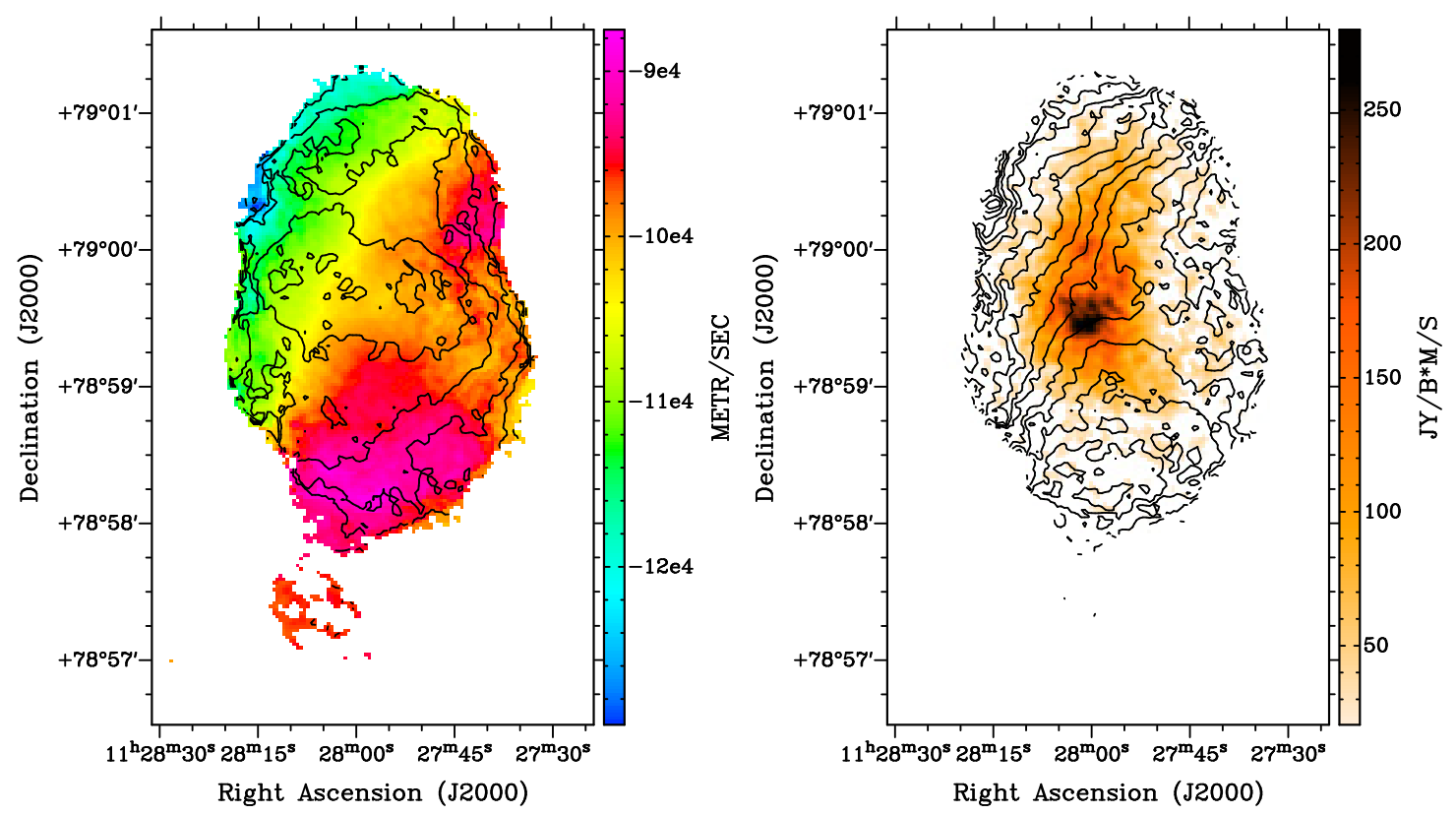

Fig. 27.- Left: The colorscale of VII Zw 403's VLA H I velocity field and contours from the VLA H I velocity dispersion map from Figure 21. Right: The colorscale of VII Zw 403's robust weighted VLA integrated H I intensity map overlaid with the velocity field contours from Figure 21.

is possible that this density enhancement is a foreground gas cloud that is disturbing the velocity field of VII Zw 403.

In an attempt to separate out emission from a potential foreground gas cloud from the rest of the disk, the AIPS task BLANK was used. BLANK allows the user to select which emission to keep in a cube by several methods; the one used here was blanking by hand: a map of each velocity channel in the data cube is displayed and the user then indicates which emission to keep by tracing out that emission. Since the potential foreground gas cloud appears at higher negative velocities than the rest of the gas in the disk in the same line of sight (see Figure 28) and since the potential foreground gas cloud is highly directional, the emission associated with the potential foreground gas cloud morphologically appears to stick out of the side of VII Zw 403's disk (to the west of the 

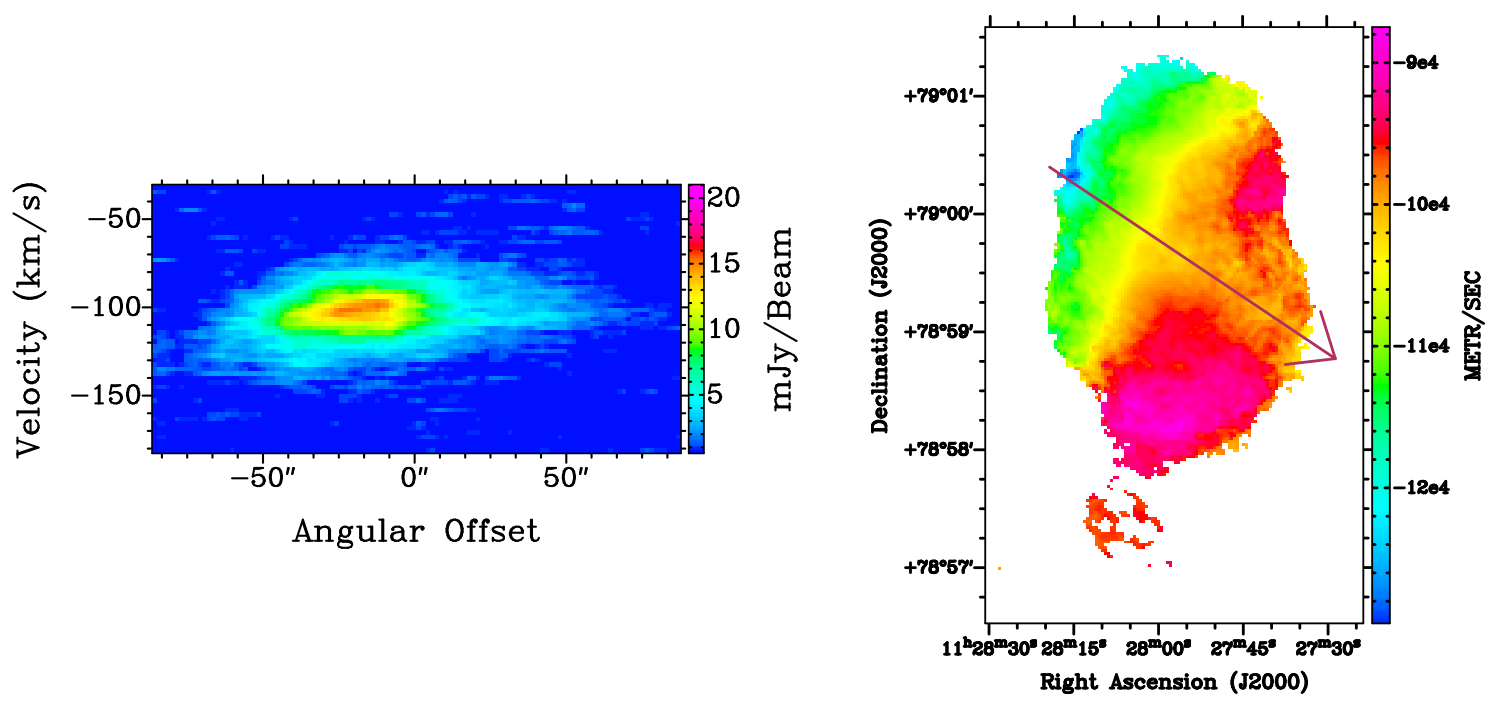

Fig. 28. - VII Zw 403: The left column contains the P-V diagrams and the right column contains the natural weighted integrated H I map with a red arrow indicating the location of the corresponding slice through the galaxy and pointing in the direction of positive offset. The slice goes through the velocity disturbance in VII Zw 403.

disk) in the data cube's individual velocity channels. Therefore, to blank the foreground gas cloud, I went through each individual velocity channel map of the galaxy and selected the emission that morphologically appears to be associated with the foreground gas cloud and not the disk of the galaxy.

The results of blanking are shown in Figure 29. The map on the top-left is the velocity field of the galaxy without most of the emission from the foreground gas cloud, the map on the top-right of the figure is the velocity field of the foreground gas cloud, and the map on the bottom of the figure compares the two by showing the contours of the first and the colorscale of the second. With most of the foreground gas cloud emission removed, the velocity field on the west side of the galaxy now generally follows the solid body trend seen on the east side of the galaxy. The emission from the foreground gas cloud also has a generally smooth transition in velocities. The mass of the foreground gas cloud emission 

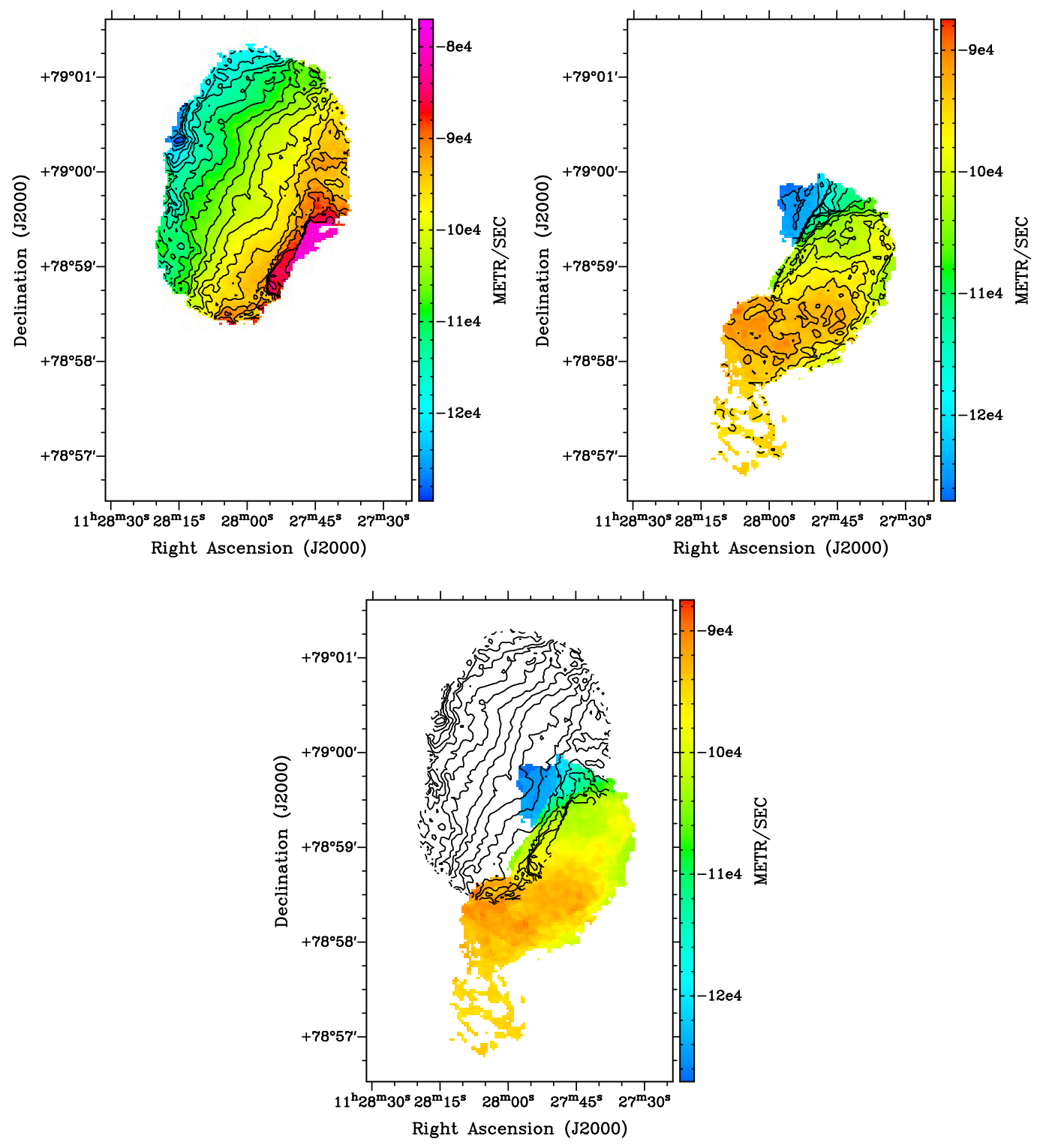

Fig. 29.- VII Zw 403: Upper Left: Intensity weighted velocity field of VII Zw 403 without the emission from foreground gas cloud. The contours are $-127.5 \mathrm{~km} \mathrm{~s}^{-1}$ to $-85.0 \mathrm{~km}$ $\mathrm{s}^{-1}$ in intervals of $2.5 \mathrm{~km} \mathrm{~s}^{-1}$. Upper Right: The intensity weighted velocity field of the foreground gas cloud. The contours are $-125.0 \mathrm{~km} \mathrm{~s}^{-1}$ to $-92.5 \mathrm{~km} \mathrm{~s}^{-1}$ in intervals of 2.5 $\mathrm{km} \mathrm{s}^{-1}$. Bottom: The contours from the velocity field in the upper left and colorscale of the velocity field in the upper right.

is $\sim 7.5 \times 10^{6} M_{\odot}$ or $18 \%$ of the total VLA H I mass measured. The length in projection of the cloud at the distance of VII Zw 403 is $\sim 4.5 \mathrm{kpc}$. 
The beginning of the disturbance of the velocity field also spatially lines up well with the curved feature seen in the robust weighted maps. The alignment of these two features can be seen in the right side of Figure 27, where the robust weighted integrated H I intensity map colorscale has been plotted with the contours of the natural weighted velocity field. Simpson et al. (2011) suggested that the curved structure seen in the robust weighted map may be a stalled hole that has broken out of the disk. If a hole has broken through front of the disk (the side closest to us), then that hole could be ejecting material into the foreground of the western side of the disk. The ejected material could then be distorting the velocity field and the velocity dispersions of the galaxy. The velocity dispersions could also in this case be disturbed on both the west and the east side of the disk since the hole could be expanding into the other side of the disk. The hole would eject material nearly perpendicular to the disk into the foreground of the west side of the galaxy, orienting the disk so that the east side is closest to us and the west side is furthest away. The rough estimates made by Simpson et al. (2011) using the McCray \& Kafatos (1987) show that this cavity would have to be made by 2800 stars with masses greater than $7 M_{\odot}$. Simpson et al. (2011) did not see evidence for this very large number of stars and suggest that the cavity may have instead been made over a long period of time or was made through consumption when the star formation rate was higher around 600-800 Myr ago. If the cavity is a hole that broke through the disk roughly 600-800 Myr ago, and the foreground gas cloud is gas that has been expelled from the disk by a now stalled hole, then the hole would require an outflow velocity of only $\sim 8 \mathrm{~km} \mathrm{~s}^{-1}$ to have moved the gas $\sim 4.5 \mathrm{kpc}$. This is a reasonable rough estimate for an outflow velocity.

The only other potential hole that was found in P-V diagrams is shown in Figure 30. The P-V diagram has a black circle outlining the potential hole. The hole is only $4.2^{\prime \prime}$ wide in diameter or $90 \mathrm{pc}$. If this is a hole, then it is unresolved by the robust synthesized beam which has a linear resolution of $200 \mathrm{pc}$. The front side of the hole (higher negative 

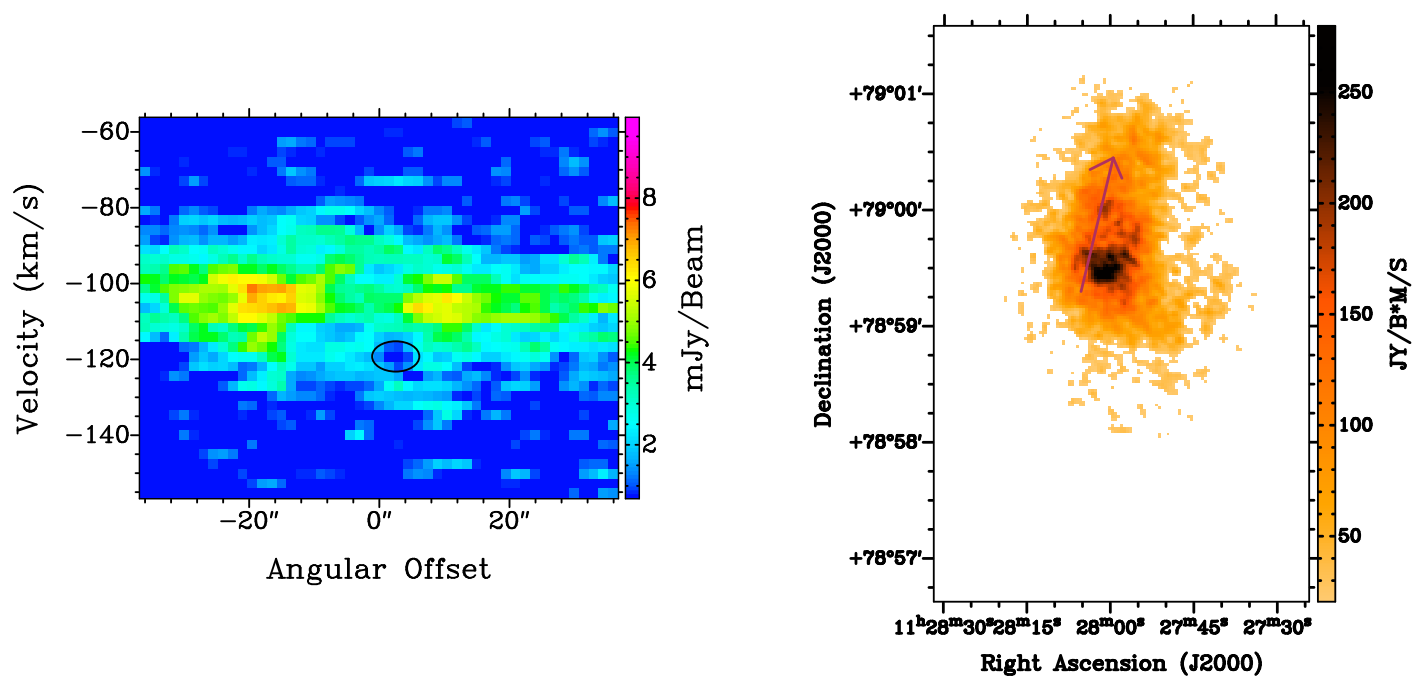

Fig. 30.- VII Zw 403: The left column contains the P-V diagram and the right column contains the robust-weighted integrated $\mathrm{H}$ I map with a red arrow indicating the location of the corresponding slice through the galaxy and pointing in the direction of positive offset. The slice goes through a potential hole (outlined in black).

velocities) appears to have some tenuous emission and does not appear to have clearly broken through the disk, however, this appearance may be the result of the hole being unresolved. Using the models from Chevalier (1974) and McCray \& Kafatos (1987) and the same assumptions as those used for Mrk 178's hole, the energy needed to make this hole is $\sim 9 \times 10^{50} \mathrm{ergs}$, and with 100 stars of stellar mass greater than $7 M_{\odot}$, the models in McCray \& Kafatos (1987) predict an age of only 2 Myr. This is a very short time to create an outflow $4.5 \mathrm{kpc}$ in length and would require very high outflow velocities of $\sim 200 \mathrm{~km}$ $\mathrm{s}^{-1}$. The unresolved hole in Figure 30 has not likely created an outflow of $4.5 \mathrm{kpc}$.

The foreground gas cloud could also be a gas cloud that is impacting the disk of VII Zw 403. With a mass of $\sim 7.5 \times 10^{6} M_{\odot}$, VII Zw 403's foreground gas cloud is a reasonable size for a starless gas cloud (Sancisi et al. 2008). The integrated H I intensity map of the foreground gas cloud is shown in Figure 31 in colorscale with the contours of the remaining disk's integrated H I intensity map. The cloud has an H I peak near central 

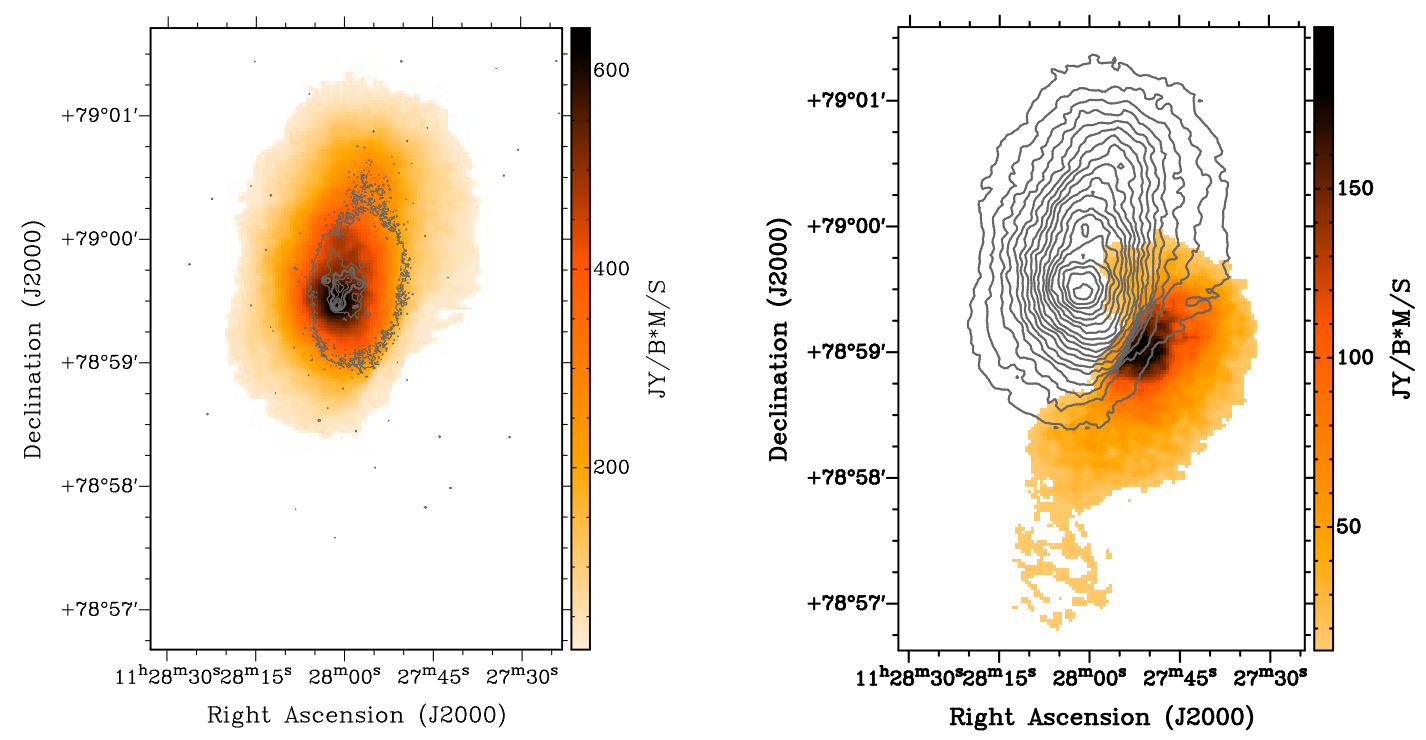

Fig. 31.- VII Zw 403: Left: The colorscale of the integrated H I intensity map of the disk without the foreground gas cloud with contours of the V-band data. Right: The colorscale of the integrated $\mathrm{H}$ I intensity map of the foreground gas cloud with contours of the integrated $\mathrm{H}$ I intensity map of the rest of the disk.

declinations. The northern end of the cloud also aligns well with the curved feature seen in the robust $\mathrm{H}$ I column density maps and also in Figure 31 as a small second $\mathrm{H}$ I peak to the north of the dense $\mathrm{H}$ I region in the remaining disk. The curved feature could then be part of the foreground gas. However, the velocity dispersions that may be associated with the northeast-southwest velocity disturbance are also on the east side of the galaxy and the foreground gas cloud does not appear to be in the foreground of the east side of the galaxy. If it was in the foreground of the east side, then it would likely also disturb the velocity field on that side. Perhaps the gas cloud is starting to run into the disk and is pushing gas on one side of the disk, which in turn, pushes gas on the other side of the disk. Models are needed to see if a gas cloud merging with a galaxy can account for the dispersions seen in this map. 
The remaining velocity field of VII Zw 403's disk is also interesting. The disk does not appear to have a kinematic major axis that is aligned with the morphological major axis of the gaseous or stellar disk. The PA of the kinematic major axis is roughly $55^{\circ}$ (estimated by eye), while the stellar morphological major axis is $-10.8^{\circ}$ (Hunter \& Elmegreen 2006). The stellar morphological axis appears to match the $\mathrm{H}$ I disk morphological axis without the foreground gas cloud well, as can be seen in Figure 31. This misalignment of axes indicates that the gaseous disk of VII Zw 403 is disturbed, possibly from past gas consumption or a past interaction. 
12. Results: IC 10

\subsection{Stellar Component}

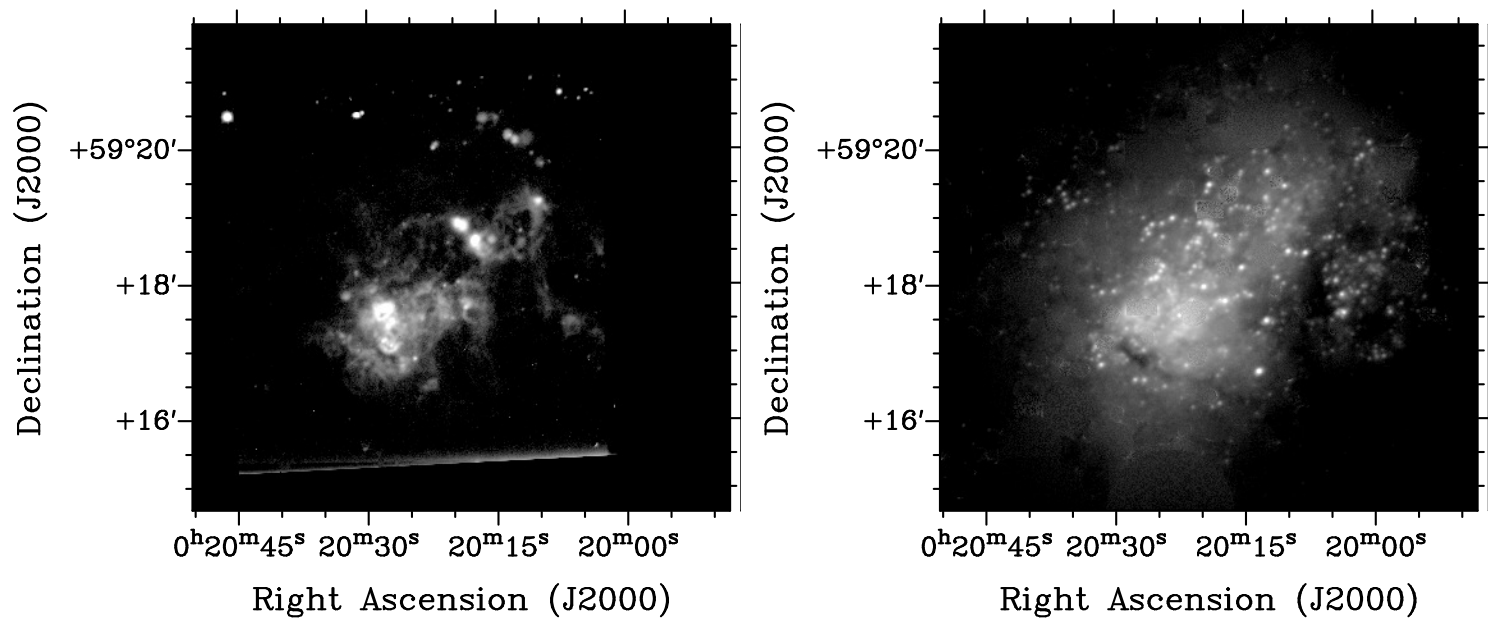

Fig. 32.- IC 10 Left: H $\alpha$; Right: V-band.

IC 10's $\mathrm{H} \alpha$ and V-band maps are shown in Figure 32. The V-band data were taken with the $1.1 \mathrm{~m}$ Hall Telescope at Lowell Observatory and the $\mathrm{H} \alpha$ data were collected with the $1.8 \mathrm{~m}$ Perkins Telescope at Lowell Observatory.

\subsection{VLA H I Morphology}

The natural weighted integrated $\mathrm{H}$ I intensity map as measured from the VLA is shown in the top-left of Figure 33. Some H I features that have been pointed out by previous authors (Wilcots \& Miller 1998; Manthey \& Oosterloo 2008) are the southern plume, the southwestern spur, the western spur, and the eastern spur. The main body of IC 10 is often considered to be the circular region of gas between all of these features. No H I column density contours are given for IC 10's VLA data because the galaxy's close proximity makes the data very high resolution and therefore too detailed for contours to be 

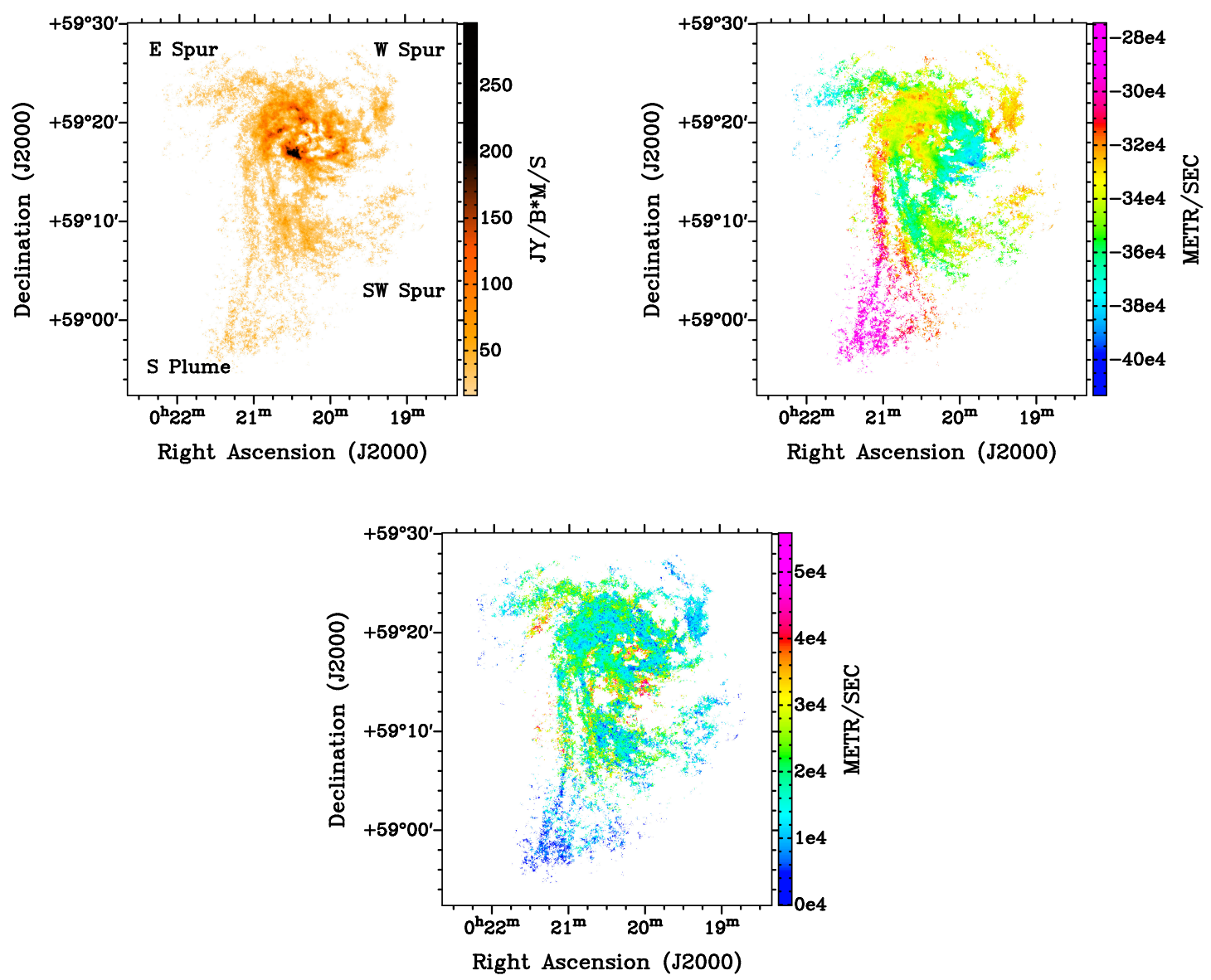

Fig. 33.- IC 10's natural-weighted moment maps. Upper left: Integrated H I intensity map blanked at a $2 \sigma$ level where $1 \sigma=14.7 \times 10^{19}$ atoms $\mathrm{cm}^{-2}$. Upper right: Intensity weighted velocity field. Bottom: Velocity dispersion field.

useful. Figure 34 shows a zoomed in image of the main body of IC 10 with the colorscale of the natural weighted $\mathrm{H}$ I column density and the contours of the stellar components.

\subsection{VLA H I Velocity and Velocity Dispersion Field}

The VLA H I velocity field is shown in the top-right of Figure 33. The main disk of IC 10 (not including the spurs or southern plume) has some regular rotation where the higher negative velocities are in the southwest and the lower negative velocities are in 

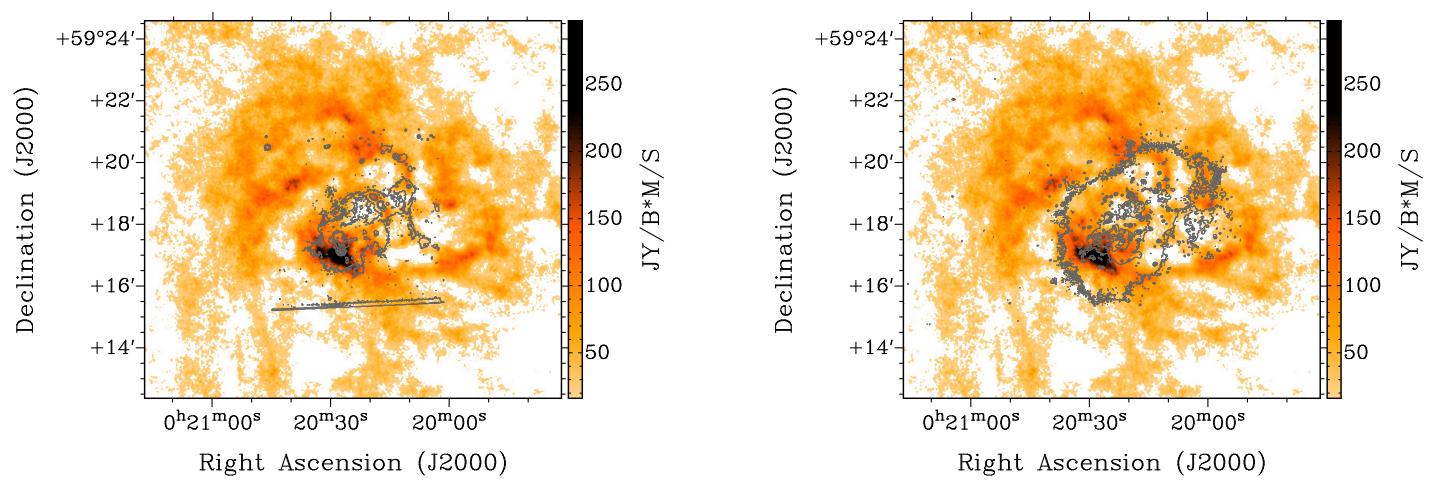

Fig. 34. - A colorscale map of IC 10's natural-weighted, main disk H I column density overlaid with stellar contours (note this map is on a different scale than those in Figure 33): Left: H $\alpha$. Right: V-band.

the northeast. The spurs do not follow the main disk's velocity trend; they have velocity gradients opposite to that of IC 10's main disk. The southern plume appears kinematically distinct from the rest of the galaxy, with the lowest negative velocities in the VLA map. The velocity dispersions, shown in the bottom of Figure 33, vary greatly.

\subsection{GBT H I Morphology and Velocity Field}

The GBT observations of IC 10 cover a $2^{\circ} \times 4^{\circ}$ area, where the longer side was set to follow the north-south direction of the southern plume seen in the VLA integrated H I intensity map (see the top-left map in Figure 33). The integrated H I intensity map as measured with the GBT is shown in the top-left of Figure 35. This first map is shown again in the top-right of Figure 35 with the $2 \sigma$ contour of the VLA data plotted on top for comparison. Another integrated H I intensity map as measured with the GBT is shown in Figure 36, where only the gas within the velocity range of -406.8 to $-373.0 \mathrm{~km} \mathrm{~s}^{-1}$ has been integrated (the full GBT H I column density map of IC 10 was made using the velocity range of -450.3 to $-228.0 \mathrm{~km} \mathrm{~s}^{-1}$ ). This velocity range was chosen to highlight a faint extension to the north of IC 10's main body. The northern extension is a new feature 

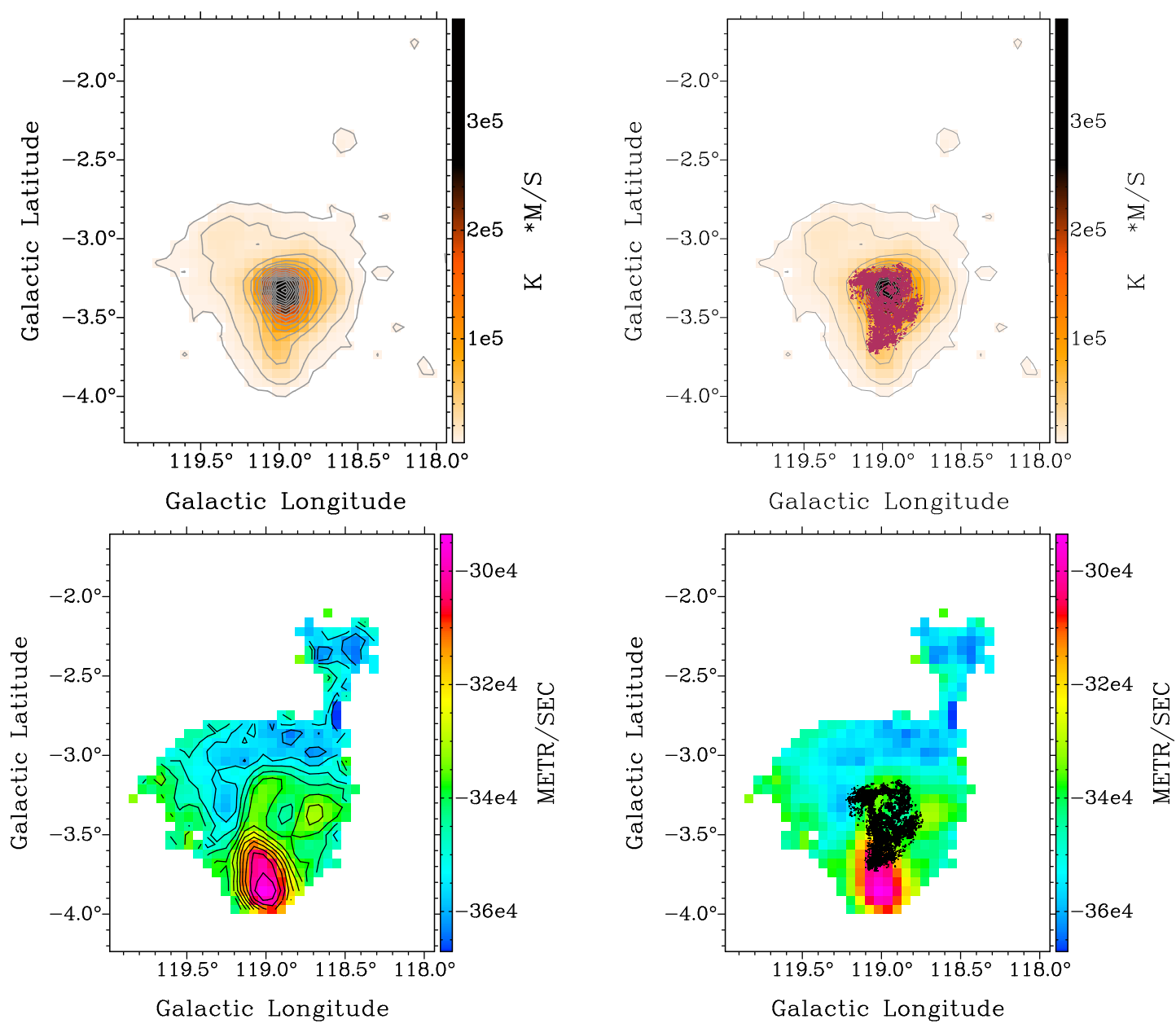

Fig. 35.- IC 10 Upper Left: GBT integrated H I intensity map; contour levels are $1 \sigma \times(8$, $20,60,100,140,180,220,260,300,340,380,420,460,500,540,580,620)$ where $1 \sigma=$ $4.76 \times 10^{17}$ atoms $\mathrm{cm}^{-2}$. Upper Right: GBT integrated $\mathrm{H}$ I intensity map from the upper left with the $2 \sigma$ contour of the VLA natural weighted column density map in red. Bottom Left: GBT H I velocity field. Contours are $-360 \mathrm{~km} \mathrm{~s}^{-1}$ to $-299.5 \mathrm{~km} \mathrm{~s}^{-1}$ separated by $5.5 \mathrm{~km} \mathrm{~s}^{-1}$. Bottom Right: The GBT velocity field in the bottom left with the $2 \sigma$ contour of the VLA natural weighted column density map in black.

not previously detected (see Nidever et al. 2013). It has a projected length of $5 \mathrm{kpc}$, where the endpoints were taken from the most northern tip of the $6 \sigma$ contour to where the disk of the main body of IC 10 and the extension meet. At the most northern end of the new extension, the column density appears to be slightly higher. 

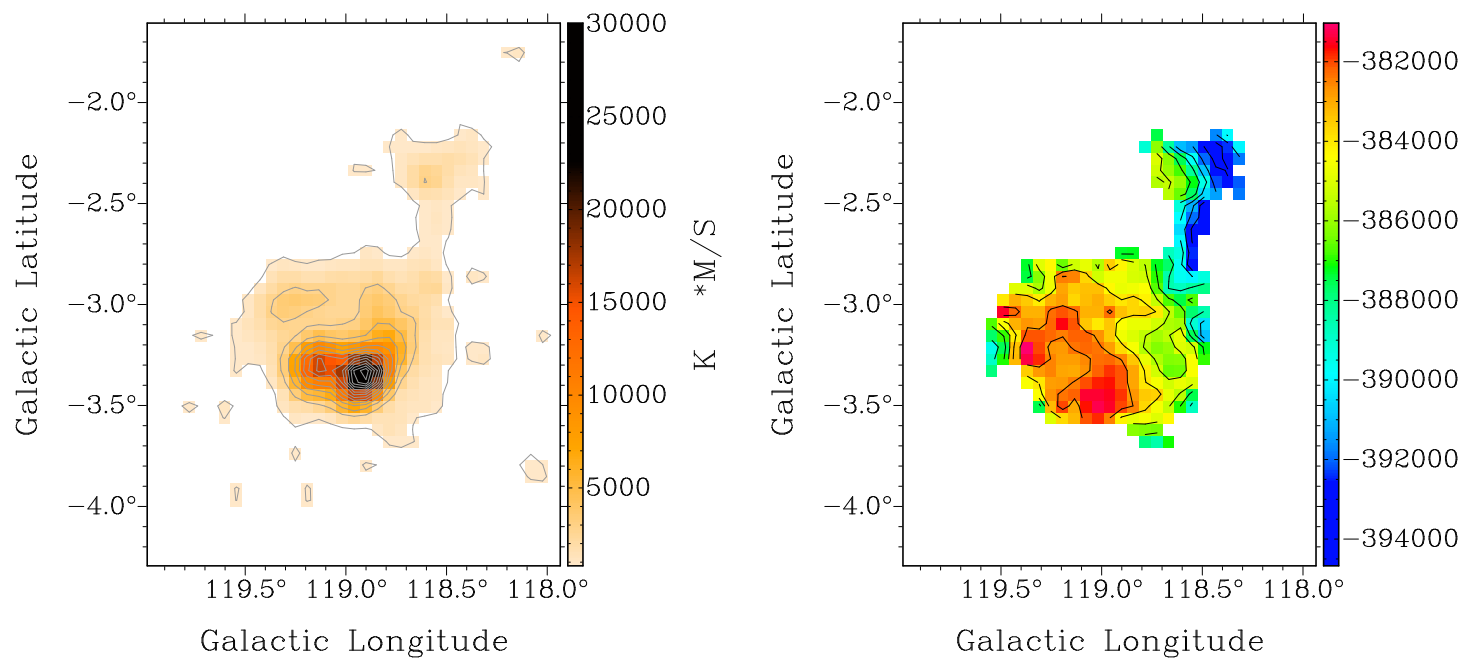

Fig. 36. - IC 10 Left: GBT integrated H I intensity map of the northern extension; the velocity range used for integration is -406.8 to $-373.0 \mathrm{~km} \mathrm{~s}^{-1}$. Contour levels are $1 \sigma \times(6,20$, $35,50,65,80,95,110,125,140,155,170,185,200)$ where $1 \sigma=1.1 \times 10^{17}$ atoms cm $^{-2}$. Right: GBT H I velocity field of the northern extension map. Contours are -393 to -382.5 $\mathrm{km} \mathrm{s}^{-1}$ separated by $1.5 \mathrm{~km} \mathrm{~s}^{-1}$.

The blanked full velocity field of IC 10's emission is shown in the bottom-left of Figure 35. Since the noise in the H I maps are also assigned a velocity, the velocity maps can become very confusing unless the velocities assigned to the noise are removed through BLANKing. The full velocity field was blanked more than once to include the emission from the main body of IC 10 seen in the top-left of Figure 35 and the emission from the northern extension seen in the top-left of Figure 36. A second map is shown in the bottom-right of Figure 35 of the colorscale of the full velocity field with the $2 \sigma$ contour of the VLA H I column density for comparison. The southern plume continues to have a distinct velocity range from the rest of the galaxy in the GBT map. Two regions (one near the center and one in the west side of IC 10's main disk) stand out in the GBT velocity field with velocities of $\sim 330-340 \mathrm{~km} \mathrm{~s}^{-1}$. The northern extension also has a distinct velocity range. Its velocities smoothly transition into the main body starting in the northwest of the main body, then over to the northeast, and finally down the east side of the main body. The northern extension occurs at velocities almost $200 \mathrm{~km} \mathrm{~s}^{-1}$ away from the Milky Way 
emission seen in the GBT maps. This indicates that it is not likely a foreground gas cloud in the Milky Way. It also smoothly transitions into the main body of IC 10 indicating that the northern extension is likely associated with IC 10. The selected velocity ranges that were used to highlight the northern extension have also been mapped and are shown in the right side of Figure 36. The part of the northern extension that is furthest north and has a round appearance has a small velocity gradient in the east-west direction of $\sim 10 \mathrm{~km} \mathrm{~s}^{-1}$. The smooth transition of velocities in the northern extension into the velocities of the main body is also more apparent in this map.

\subsection{H I Mass}

The total H I mass from the VLA data is measured to be $5 \times 10^{7} M_{\odot}$ and the total mass of the GBT data was measured to be $10^{8} M_{\odot}$. The VLA was able to recover $50 \%$ of

the GBT mass. The mass of the northern extension is also measured to be $10^{6} M_{\odot}$ or $1 \%$ of the total GBT mass. The area used to measure the mass of the northern extension is where the northern extension meets the main body of IC 10 in the GBT integrated H I intensity map to the top of the $6 \sigma$ contour level of the H I map in Figure 36.

\section{Discussion: IC 10}

The morphology and kinematics of the gas in IC 10 is complex as shown in both the GBT and VLA images. The new extension seen in the GBT H I data may reveal what has happened to IC 10 in the past. In this section I will discuss three possible explanations for IC 10's H I morphology and kinematics. 


\subsection{A Galaxy Still in Formation}

Shostak \& Skillman (1989) and Wilcots \& Miller (1998) suggested that IC 10 may be a galaxy still in the process of forming by passively accreting the surrounding IGM. Since the northern extension extends well beyond the disk and is associated with the disk, then the northern extension would likely be gas that is being accreted onto IC 10 if it is a galaxy still in formation. In the right side of Figure 36, the velocity field of IC 10's new extension shows that the parts of the northern extension that are further from the main body of IC 10 have progressively larger negative velocities. These velocities are also increasing away from the systemic velocity of IC 10 , which is $-349 \mathrm{~km} \mathrm{~s}^{-1}$. Kereš et al. (2005) model accretion onto low mass galaxies and finds that the main mode of accretion is cold accretion through dense, cold filaments. Brooks et al. (2009) also find that cold accretion is a dominant for. of IGM accretion in low mass galaxies and suggests that the filaments will accrete onto the galaxy essentially in free fall. In this case, gas that is passively accreted onto a galaxy from the IGM would be expected to have velocities that increase as the gas gets closer to the disk.

Energy conservation was used to get a rough estimate of how much the velocity would increase along the length of the northern extension. A point mass potential was assumed as a rough estimate for the gravitational potential well. The mass of IC 10's disk was taken to be the dynamical mass of the LITTLE THINGS VLA data; $2.6 \times 10^{8} \mathrm{M}_{\odot}(\mathrm{Oh}$, et al. in prep). The expected velocity change in the filament was found to be $\sim 10 \mathrm{~km} \mathrm{~s}^{-1}$. This is a small change in velocity across the $5 \mathrm{kpc}$ length of the northern extension. The northern extension is also very elongated north of IC 10's main body. It is therefore possible that if there is an increase in velocity along the extension, then most of the velocity increase will be towards the south, in the plane of view. Therefore, the increase in velocity along the filament may not be measurable because motion in the plane does not produce a Doppler 


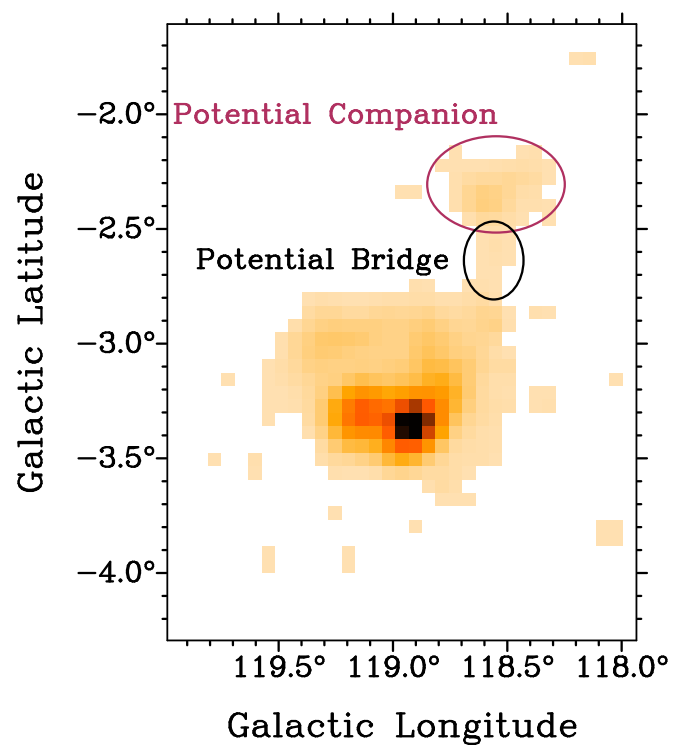

Fig. 37.- A labeled version of Figure 36 showing the location of the features in the case that the northern extension is a bridge and companion galaxy.

shift. It is thus possible that the northern extension is an intergalactic gas filament being accreted onto IC 10.

\subsection{A Galaxy with a Northern Bridge}

One possible explanation for the northern extension is a bridge (Nidever et al. 2013), where the round region at the end of the northern extension is a companion to IC 10 (see Figure 37). If the round region at the end of the northern extension is a companion, then it would likely have a stellar component. DSS ${ }^{19}$ and NED produce no known stellar component in the end of the northern extension. This does not exclude the round region as a companion; the stellar component may be too faint to be picked up in DSS.

If there is a bridge, then did it originate from the potential companion or IC 10? In the bottom-left of Figure 35, the potential bridge material appears to transition into IC 10's

\footnotetext{
${ }^{19}$ The Digitized Sky Survey http://archive.stsci.edu/cgi-bin/dss_form
} 
main body smoothly in a counterclockwise direction. In the VLA map in Figure 33, the spurs have a sharp, curved appearance indicating that the spurs are likely tidal features. The velocity gradients of the spurs are also opposite to that of IC 10's main body in the VLA maps, which suggests that IC 10's main disk in the VLA maps is not likely the parental disk of the spurs since tidal features do not counterrotate to their parental disk. The spurs also morphologically appear to be turning in a counterclockwise direction away from IC 10's main disk. Counterclockwise is the same direction that the northern extension appears to transition into IC 10's main body. Since the spurs did not likely originate from IC 10's main disk, are tidal in appearance, and morphologically turn in the same direction as the potential bridge, it is likely that they are part of the potential bridge. The potential bridge would then also have to be coming from the companion. However, tidal forces are two-sided; if a bridge is created on one side of a galaxy, then a tidal tail will be produced

on the other side (Toomre \& Toomre 1972; Barnes 1988; Mihos \& Hernquist 1996; Mihos 2004; Cox et al. 2008). Yet, there is no tidal tail associated with the potential companion, making this scenario is unlikely.

\subsection{An Advanced Merger}

A detailed investigation of the individual velocity channels of IC 10's GBT data cube, provides another possible explanation for IC 10's morphology and kinematics; IC 10 may be an advanced merger and the northern extension and southern plume may be tidal tails from that ongoing merger (see Figure 38). In Figure 36 there are two central H I peaks in the main body of IC 10, separated by a projected distance of $\sim 3 \mathrm{kpc}$. The distance between the two peaks was calculated from the distance between the two brightest pixels in the peaks from the H I column density map in Figure 36. These two peaks are shown more clearly in Figure 39, where every 5th channel of the GBT data cube has been plotted 


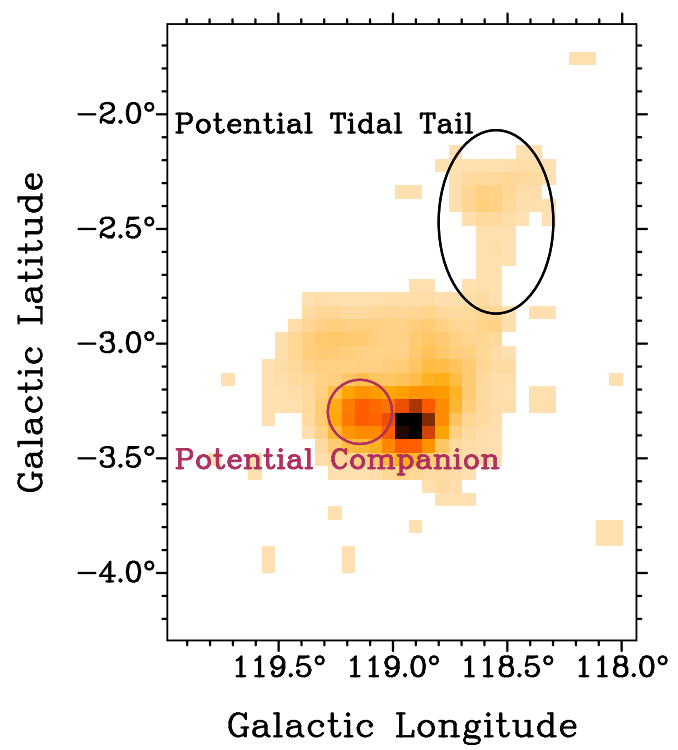

Fig. 38.- A labeled version of Figure 36 showing the location of the features in the case that the northern extension is a tidal tail and the eastern H I peak is a companion.

from $-397.1 \mathrm{~km} \mathrm{~s}^{-1}$ to $-373 \mathrm{~km} \mathrm{~s}^{-1}$. The peak to the west is associated with the $\mathrm{H}$ I main disk imaged by the VLA and the central H I peak seen in the fully integrated H I column density GBT map in Figure 35. The peak to the east, however, does not appear in the VLA map. The eastern peak may be the remnants of a companion galaxy that is merging with IC 10 and the northern extension a tidal tail that has resulted from this merger.

To see if the two peaks are kinematically distinct, a P-V diagram with a slice going through both peaks was made and shown in Figure 40. The western peak is the brightest emission in the $\mathrm{P}-\mathrm{V}$ diagram. The overall trend in the western peak is the same as that seen in the VLA data; lower negative velocities on the left side and higher negative velocities on the right side. While the eastern peak is the faint emission at $-390 \mathrm{~km} \mathrm{~s}^{-1}$ to $-350 \mathrm{~km}$ $\mathrm{s}^{-1}$ and $-1.5^{\prime}$ to $-13^{\prime}$. The eastern peak shows up below the left side of the western peak. This difference in the $\mathrm{P}-\mathrm{V}$ diagram indicates that the eastern peak is not rotating in a solid body manner with the western peak. 


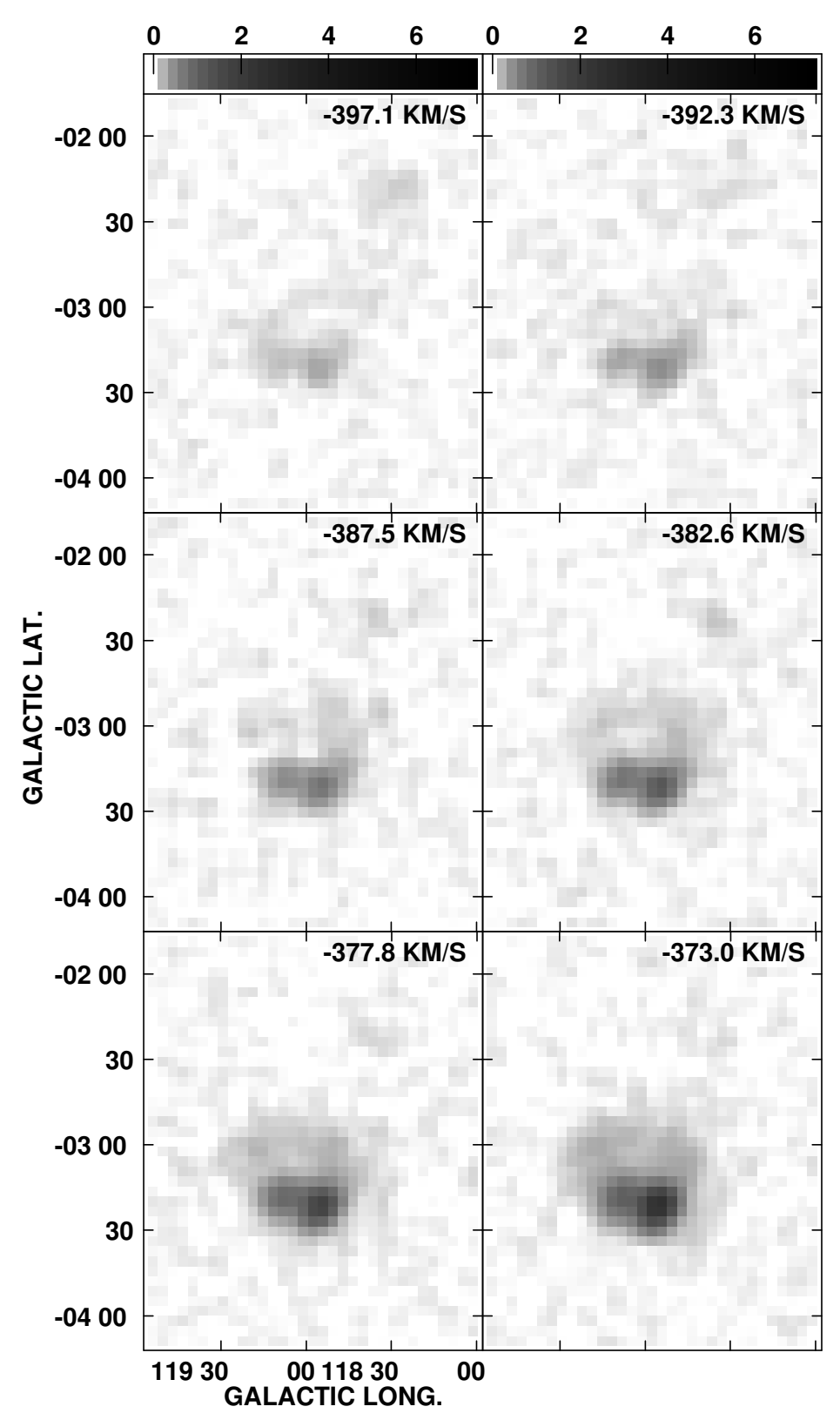

Fig. 39.- Velocity channels from IC 10's GBT data cube that clearly show the eastern peak. In the velocity range shown, these channels are every fifth channel in the data cube.

To investigate if the kinematics of the rest of the GBT H I data support an advanced merger, a new velocity map was made to exclude the western H I peak and the southern plume of IC 10. This was done in two steps: first the data cube was blanked by hand in AIPS using BLANK in an attempt to remove as much of the western peak as possible and 

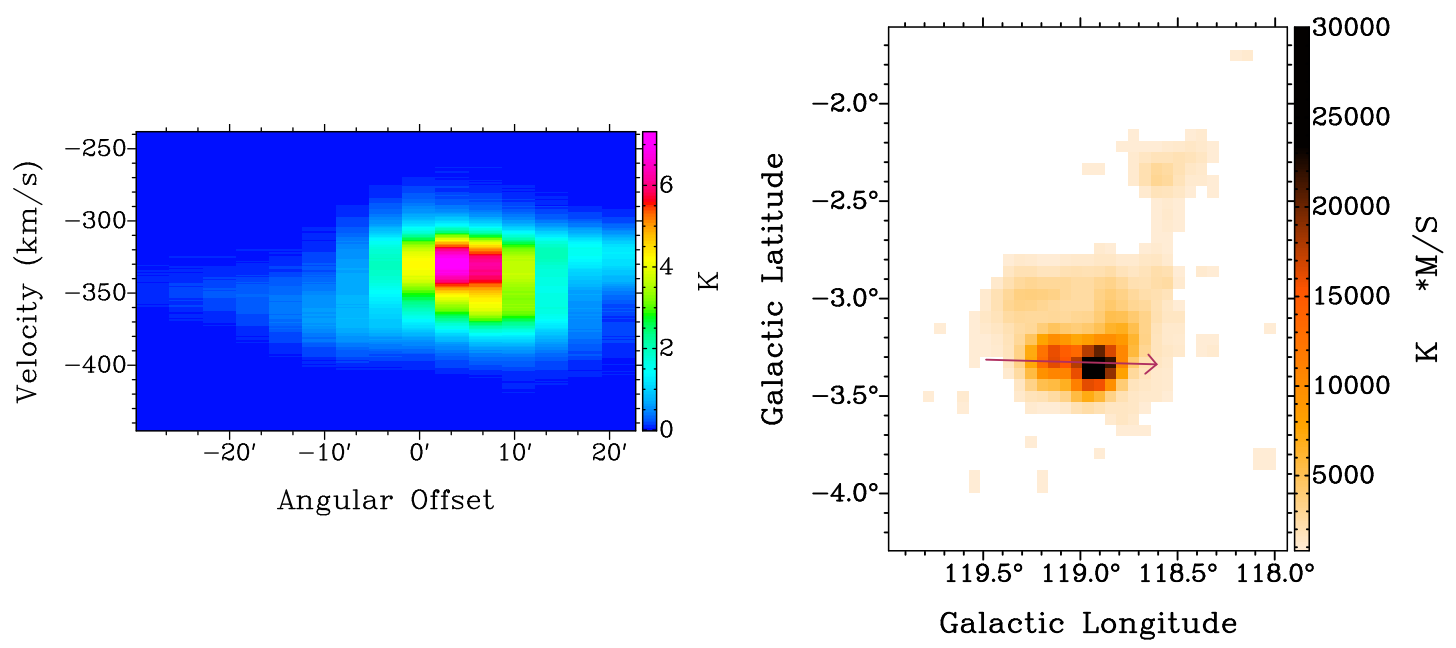

Fig. 40.- IC 10: The left column contains the P-V diagram and the right column contains the GBT integrated H I map from Figure 36 with a red arrow indicating the location of the corresponding slice through the galaxy and pointing in the direction of positive offset. This slice goes through both central peaks seen in the GBT data.

second, a velocity range of $-422.2 \mathrm{~km} \mathrm{~s}^{-1}$ to $-330.4 \mathrm{~km} \mathrm{~s}^{-1}$ was used to exclude the southern plume when integrating the channels to make a new map of the GBT H I data. The first BLANKing step was done by visual inspection of individual velocity channel maps; since the gas associated with the western peak has a distinct velocity gradient in the data cube (see Figure 40) and has a high flux, much of its emission was distinguishable from other emission in the individual velocity channel maps.

The resulting velocity field is shown in Figure 41. In this figure the velocities from the northern extension transition into the main body from the northwest to the northeast, then could spiral counterclockwise in towards the center of the galaxy. Much of the velocity field in Figure 41 could be tracing a tidal tail spiraling in towards one of the two central peaks. Depending on the properties of the interaction and the two galaxies interacting, a tidal tail can appear to be spiraling as a merger occurs. For example, in Cox et al. (2008), their Figure 4 shows the resulting time steps of a merger between two galaxies with a total mass ratio of 2.3:1 resulting in many spiraling tidal features in the gas component. The lower mass galaxy also quickly merges with the larger mass galaxy prior to the tidal tails 


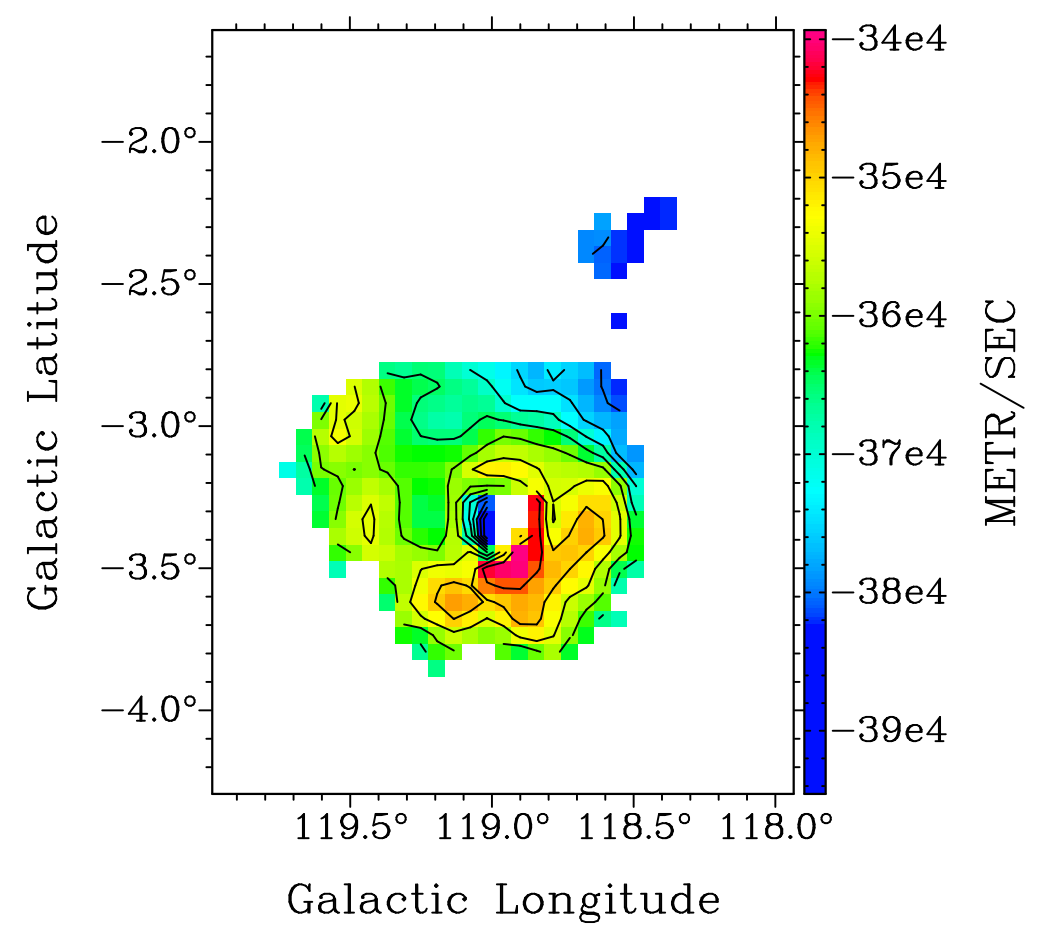

Fig. 41.- Velocity field of IC 10's GBT data without the southern plume or most of the western peak.

dissipating. It is therefore possible that IC 10 is in the process of merging with a low mass companion, and the second peak in the GBT data is what is left of IC 10's companion.

The spurs seen in IC 10's data may then be a result of the tidal tail passing closely around the western peak (the main disk of IC 10 seen in the VLA data). The spurs have velocity gradients opposite of IC 10's VLA main disk, and are therefore unlikely to have come from it. Together they also appear to morphologically point in a counterclockwise direction towards the eastern peak. Their morphological appearance and distinct velocity gradients indicate that the spurs could be part of a tidal tail that begins in the northern extension and spirals inward, originating from IC 10's potential companion in the eastern peak.

The gradient across the top round part of the northern extension can also be explained through a tidal tail. The gradient at the end of the northern extension shows a turn around 


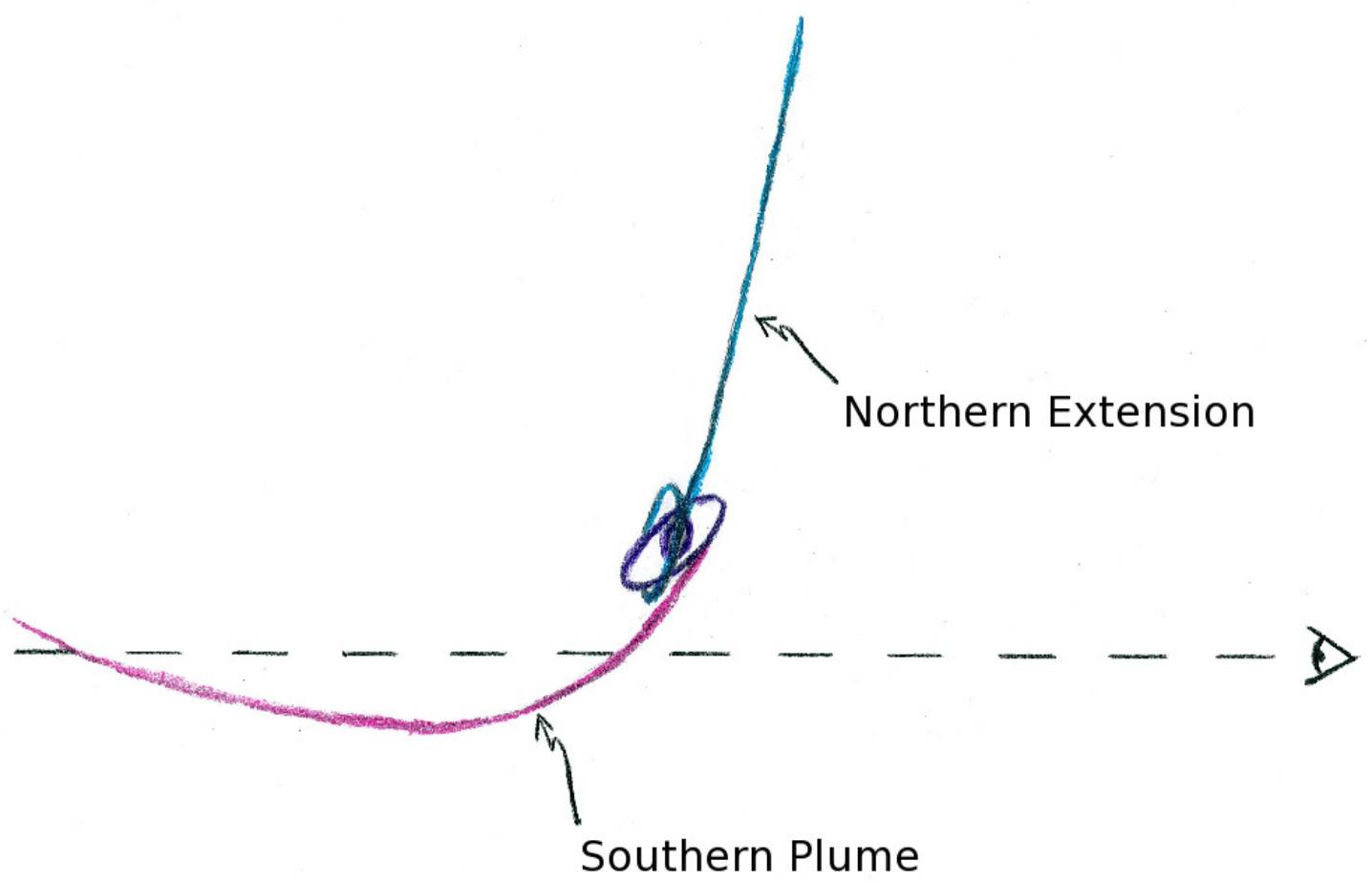

Fig. 42.- A schematic of a potential viewing angle of IC 10.

in velocity back towards lower negative velocities. The systemic velocity of IC 10 is also at a lower negative velocity of $-348 \mathrm{~km} \mathrm{~s}^{-1}$. The gradient at the end of the northern extension could be the gas at the end of the tidal tail beginning to fall back towards the galaxy.

The southern plume could also be a second tidal tail seen at an extreme projection angle. A schematic is shown in Figure 42 to demonstrate a possible viewing angle of the configuration. If the southern plume is a tidal tail that is extending along the line of sight, then it will also likely have a large velocity gradient. In Figure 43, every twelfth velocity channel containing obvious southern plume emission from the GBT data cube has been plotted. The channels plotted here cover a large amount of velocity space; $42 \mathrm{~km} \mathrm{~s}^{-1}$. The southern plume grows in vertical length in the first three channels plotted, and then becomes shorter in length in the last three channels. It also has a lower column density in 
the last two plotted channels. These features are consistent with a curved elongated feature extending along the line of sight. As seen in the VLA velocity map in the top-right of Figure 33, the velocities of the southern plume transition well into the main disk of IC 10; as the southern plume gets closer to the main disk in IC 10's VLA velocity map, its velocities become higher negative numbers and it reaches the velocities of the redshifted, east side of IC 10's main disk as it meets it in the line of sight. Therefore, if the southern plume is a tidal tail, it is likely to have originated from IC 10's VLA main disk (the western peak in the GBT map). This would make IC 10 and its possible companion galaxy similar to the antennae and mouse galaxies where each galaxy has produced a tidal tail, but at a different observing angle.

The H I velocity dispersion map, as measured by the VLA fits well with the theory of IC 10 being an advanced merger. There are generally high velocity dispersions of $\sim 20$ $\mathrm{km} \mathrm{s}^{-1}$ throughout the main disk of IC 10 indicating that the galaxy is quite disturbed, as would be expected for an advanced merger. The southern spur also has significantly higher velocity dispersions (20-30 $\mathrm{km} \mathrm{s}^{-1}$ ) where, as seen in the schematic in Figure 42, the southeastern spur would likely be crossing behind the southern plume. A jump in velocity dispersion would be expected for two features of different velocity ranges crossing each other in the line of sight. The southern spur then has lower velocity dispersions as it gets further from the main disk of IC 10. The spurs have this same pattern of low velocity dispersions further from the main disk. Low velocity dispersions are not unusual for tidal tails. Therefore, the higher velocity dispersions in the spurs closer to the disk could be caused by the gas in the spurs closer to the main disk overlapping with some of the extended emission from the main disk.

The stellar kinematics of IC 10 were observed by Gonçlaves et al. (2012) by measuring the motion of planetary nebulae $(\mathrm{PNe})$ with ages of $\sim 8 \mathrm{Gyr}$. They concluded that the kinematics of these PNe followed roughly the kinematics of the VLA H I maps 


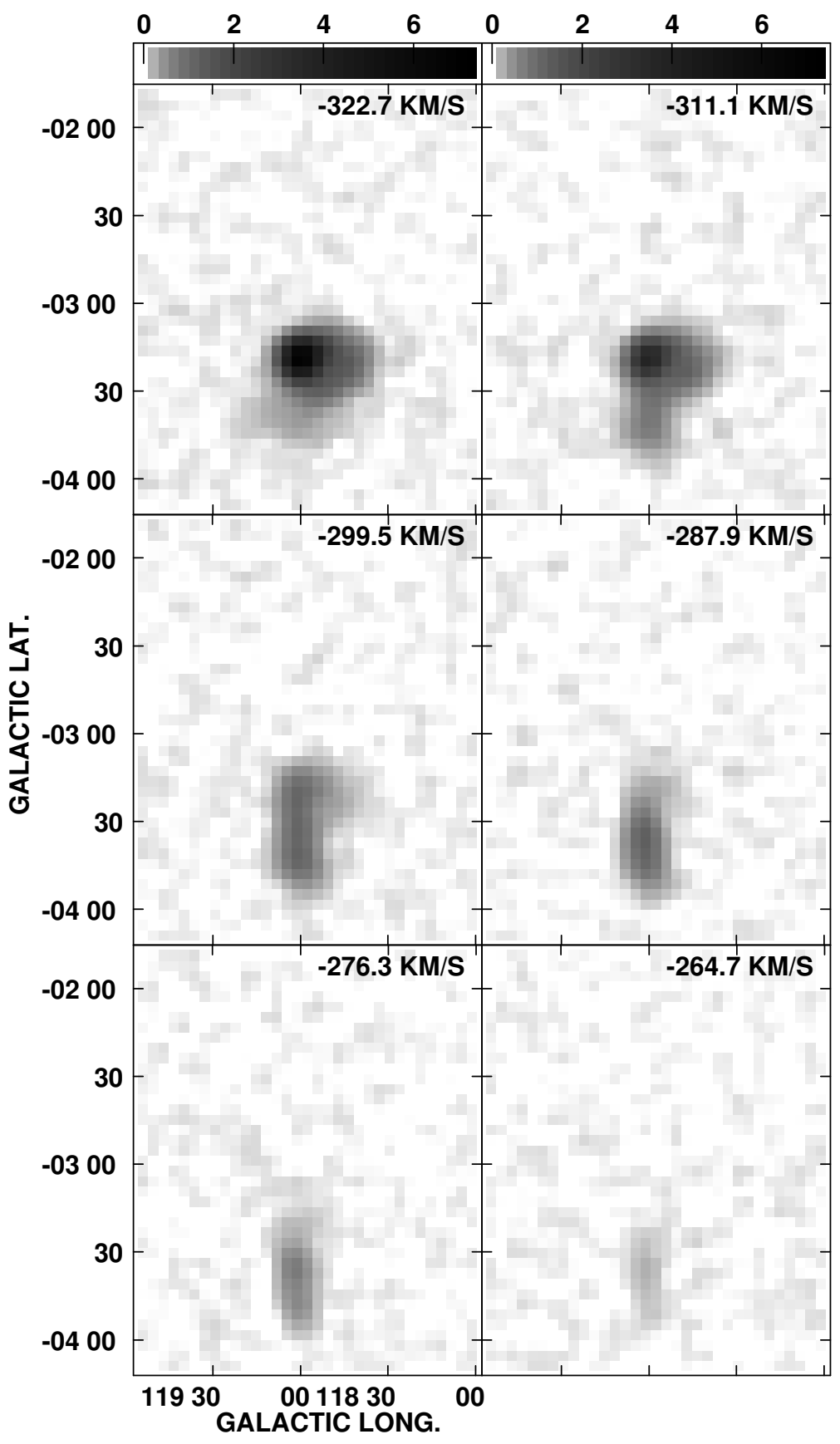

Fig. 43.- Velocity channel maps for IC 10's southern plume at intervals of 12 channels.

from Wilcots \& Miller (1998), including the western spur. Some of these PNe at large radii may therefore be a part of the spiraling tidal feature where the PNe are stars that have been pulled from the outskirts of the potential companion in the eastern peak. The only 
stellar components found in DSS and NED near the eastern peak were labeled as part of IC 10. However, no obvious stellar disk was seen in the location of the eastern peak in DSS. Deeper stellar images of this region are needed to confirm if there is a stellar component to the eastern peak. 
14. Results: NGC 3738

\subsection{Stellar Component}
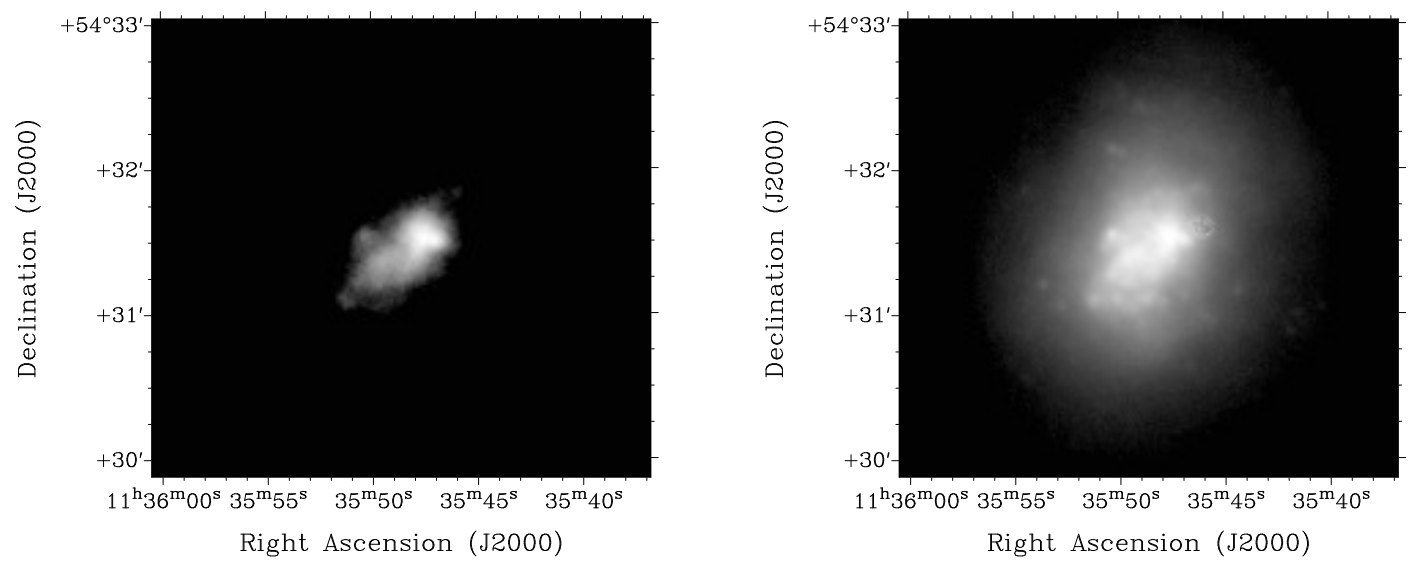

Fig. 44.— NGC 3738 Left: FUV.; Right: V-band.

The FUV and V-band data for NGC 3738 are shown in Figure 44. The V-band data were taken with the $1.1 \mathrm{~m}$ Hall Telescope at Lowell Observatory and the FUV were taken with GALEX. The FUV and V-band data have very similar morphology.

\subsection{VLA H I Morphology}

NGC 3738's natural weighted integrated H I intensity map as measured by the VLA is shown in the top-left of Figure 45. There are several pieces of emission surrounding the disk. The robust weighted map is shown in the top-left of Figure 46. The top-right and bottom of Figure 46 show the colorscale of the robust weighted, integrated H I intensity map and contours of the FUV and V-band data, respectively. The brightest FUV region coincides with the region of high $\mathrm{H}$ I column density. The V-band data stretch beyond the gaseous disk in the natural and robust weighted maps. 

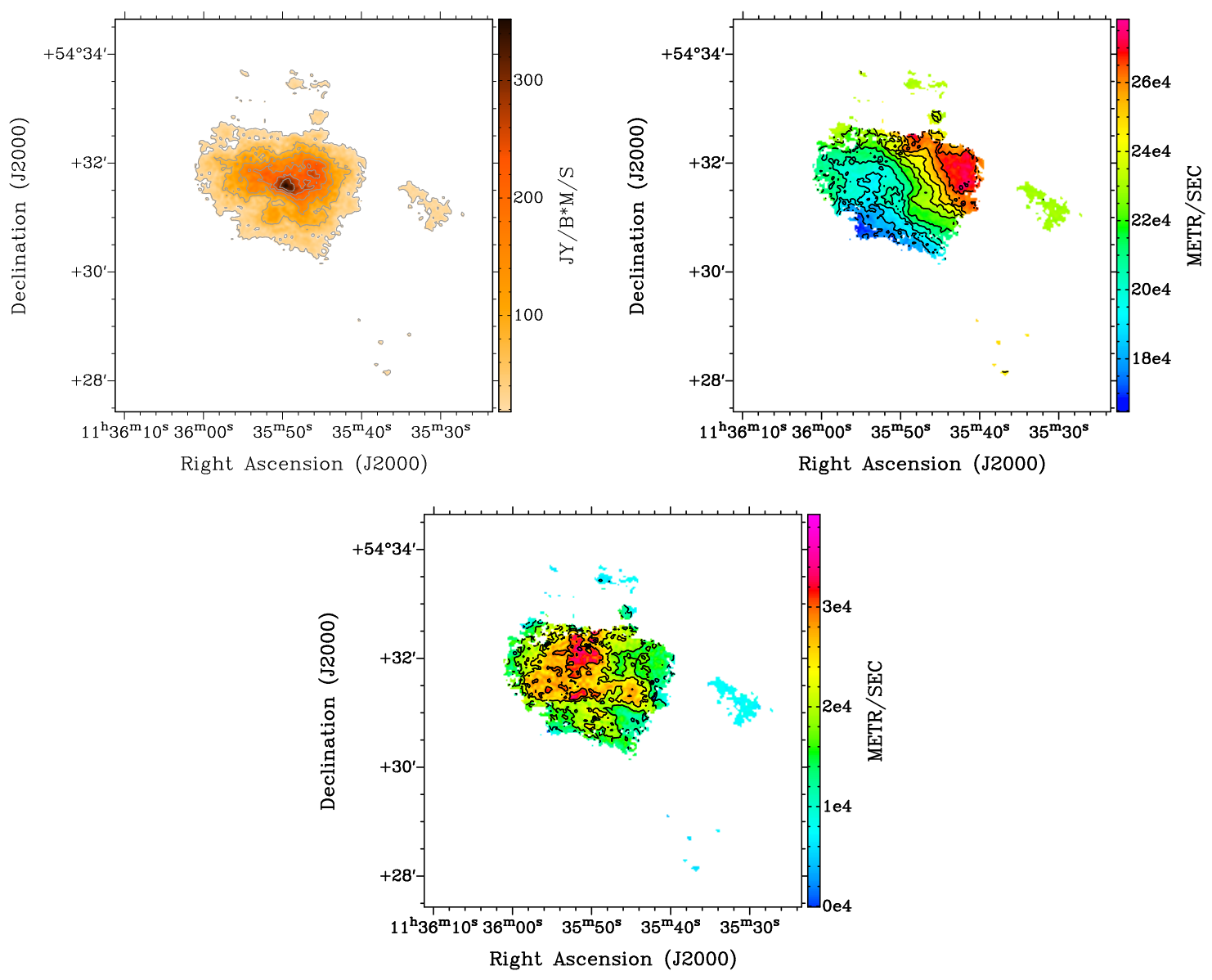

Fig. 45.—NGC 3738's natural-weighted moment maps. Upper left: Integrated H I intensity map; contour levels are $1 \sigma \times(2,8,14,20,26,32)$ where $1 \sigma=1.01 \times 10^{20}$ atoms cm $\mathrm{cm}^{-2}$. Upper right: Intensity weighted velocity field; contour levels are $165 \mathrm{~km} \mathrm{~s}^{-1}$ to $275 \mathrm{~km} \mathrm{~s}^{-1}$ separated by $10 \mathrm{~km} \mathrm{~s}^{-1}$. Bottom: Velocity dispersion field; contour levels are $5 \mathrm{~km} \mathrm{~s}^{-1}$ to $35 \mathrm{~km} \mathrm{~s}^{-1}$ separated by $5 \mathrm{~km} \mathrm{~s}^{-1}$.

\subsection{VLA H I Velocity and Velocity Dispersion Field}

The velocity field of the $\mathrm{H} \mathrm{I}$ is shown in the top-right of Figure 45. The disk appears to be participating in solid body-like rotation, with some small kinks in the velocity field. The velocities of the separate pieces of emission that surround the disk are all near the systemic velocity of the galaxy of $229 \mathrm{~km} \mathrm{~s}^{-1}$ indicating that they are likely associated with NGC 3738. The velocity dispersion field is shown in the bottom of Figure 45. Velocity dispersions reach $\sim 20 \mathrm{~km} \mathrm{~s}^{-1}$ and above throughout most of the disk. 

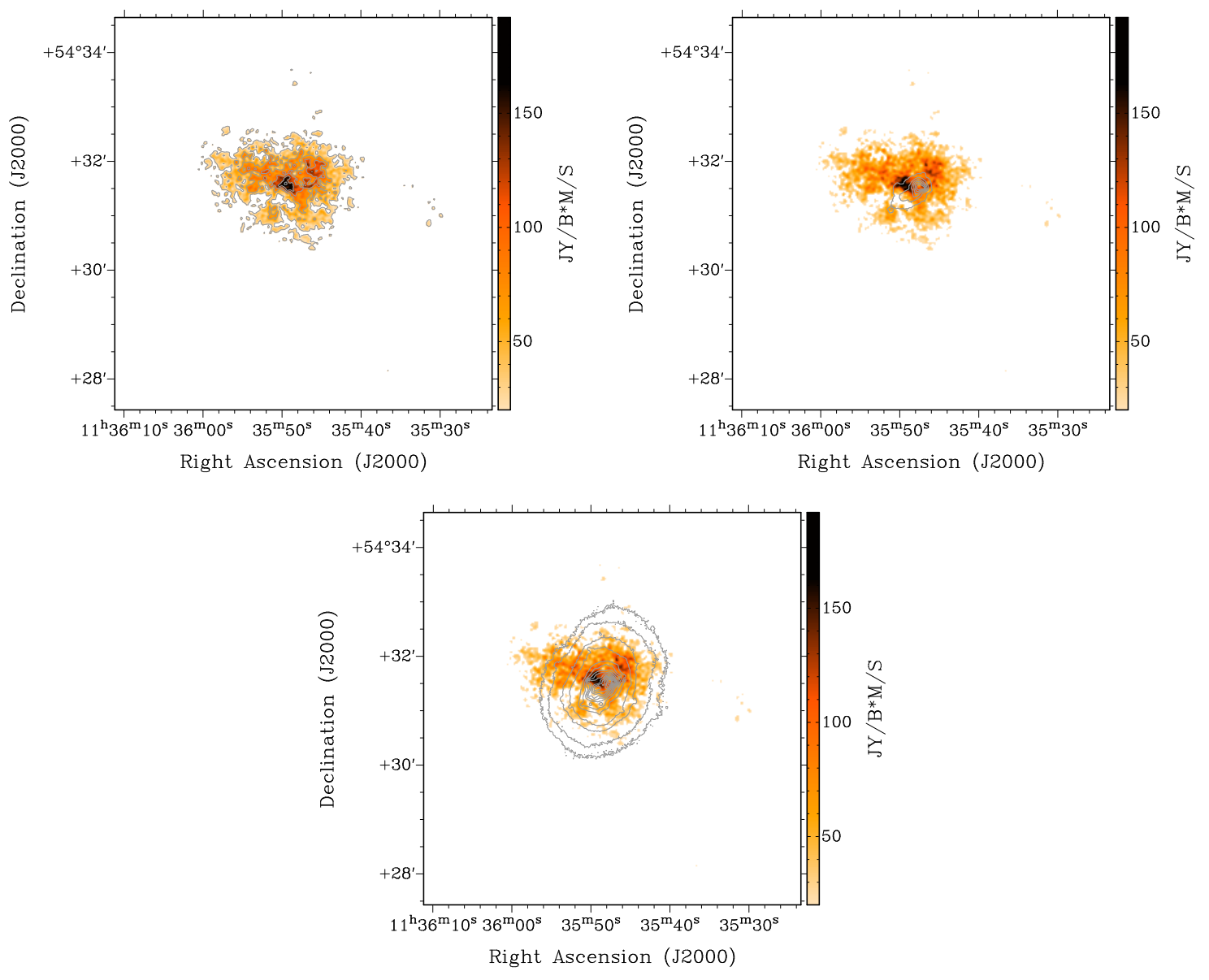

Fig. 46. - NGC 3738's natural-weighted moment maps. Upper left: Integrated H I intensity map; contour levels are $1 \sigma \times(2,6,10,14,18)$ where $1 \sigma=3.27 \times 10^{20}$ atoms cm $^{-2}$. Upper right: Integrated $\mathrm{H}$ I intensity map colorscale and FUV contours. Bottom: Integrated H I intensity map colorscale and V-band contours.

\subsection{GBT H I Morphology and Velocity Field}

The integrated H I intensity map as measured with the GBT is shown in Figure 47. NGC 3738 is at the center of the map surrounded by some noise and part of a galaxy in the top left of the map. The velocity field of the GBT H I data is shown in the right side of Figure 47. There is a gradient evident in NGC 3738's emission from bottom left to top right in this coordinate system, which matches the gradient direction seen in the 

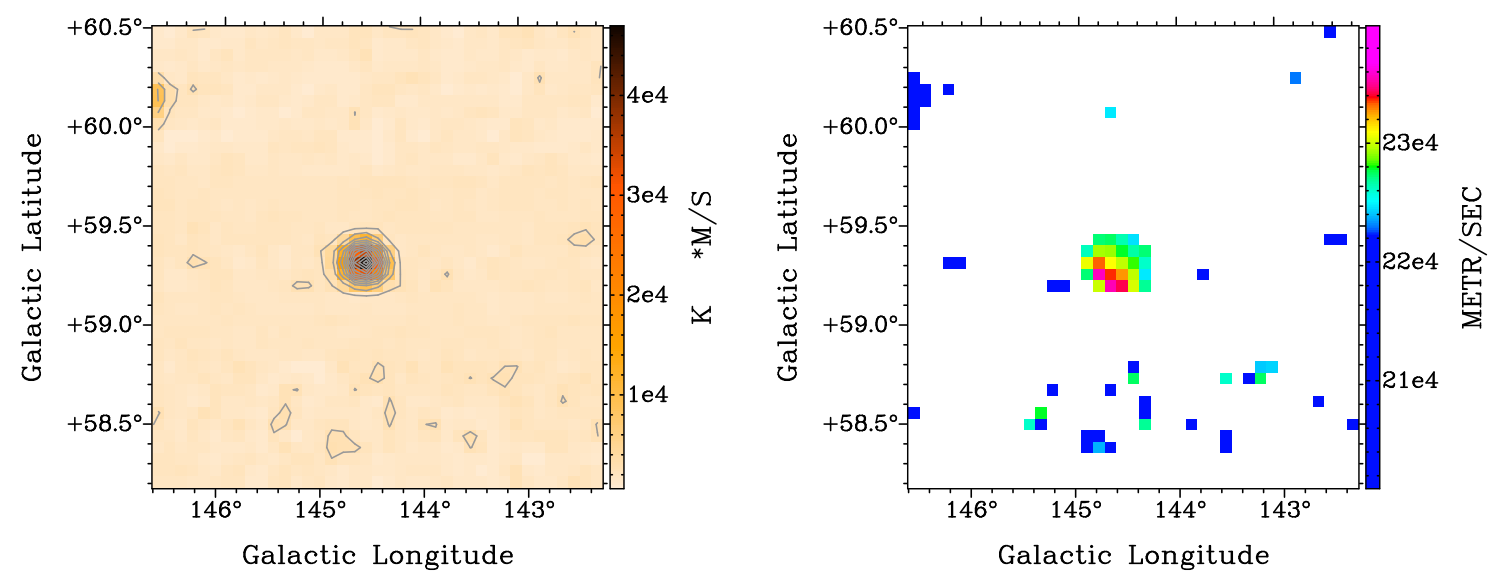

Fig. 47.- NGC 3738's GBT moment maps. Left: Integrated H I intensity map; contour levels are $1 \sigma \times(7,17,27,37,47,57,67,77,87,97,107,117,127)$ where $1 \sigma=2.731 \times$ $10^{17}$ atoms $\mathrm{cm}^{-2}$. Right: Intensity weighted velocity field.

VLA maps which are in RA and Dec. NGC 3738 does not appear to have any nearby companions.

\subsection{H I Mass}

The total H I mass of NGC 3738 measured from the VLA emission, including the separate pieces of emission, is $9.5 \times 10^{7} M_{\odot}$, and the total $\mathrm{H}$ I mass measured from the GBT emission for NGC 3738 is $1.7 \times 10^{8} M_{\odot}$. The VLA was able to recover $56 \%$ of the GBT mass. The masses of the individual pieces of gas external to the disk in NGC 3738's VLA data add up to $1.3 \times 10^{7} M_{\odot}$ or $14 \%$ of the total VLA mass.

\section{Discussion: NGC 3738}

From the VLA data, NGC 3738 is morphologically and kinematically disturbed, including high velocity dispersions throughout most of the disk. The small pieces of emission around the disk of NGC 3738 could be noise in the map. A map convolved with 


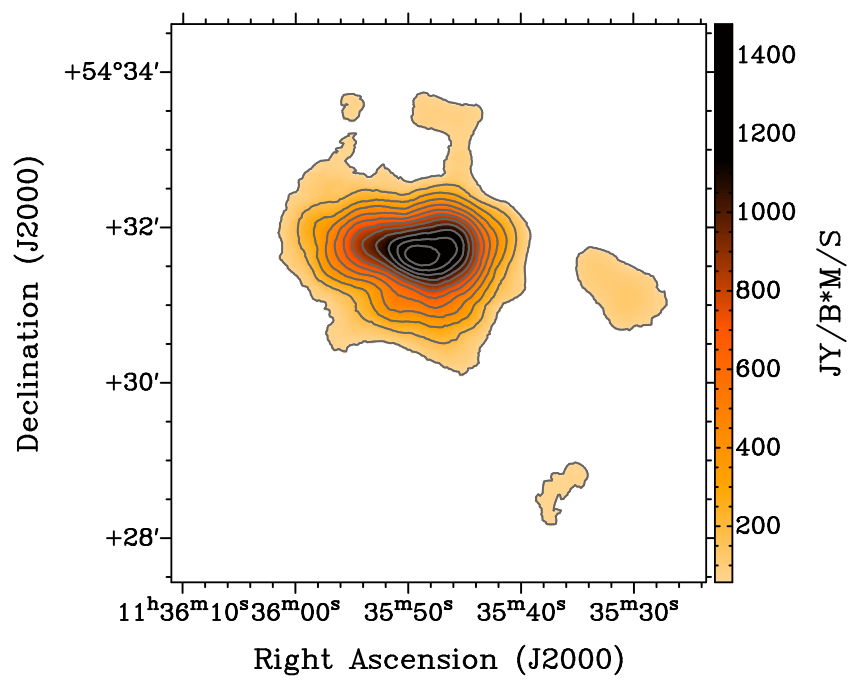

Fig. 48. - NGC 3738's $25^{\prime \prime} \times 25^{\prime \prime}$ convolved map.

a $25^{\prime \prime} \times 25^{\prime \prime}$ beam was made to see if any more faint emission from these clouds could be picked up by boosting the signal-to-noise. The resulting integrated $\mathrm{H}$ I intensity map is shown in Figure 48, where the clouds are still present and larger. These clouds are likely real emission.

With several pieces of separate emission surrounding the disk, it is possible that the high dispersions are due to a foreground gas cloud(s) projected onto the disk of NGC 3738. P-V diagrams were made to search the disk for kinematically distinct clouds. A P-V diagram showing the one kinematically distinct gas cloud that was found is shown in Figure 49. The overall trend seen in the gas through this slice is an increase in velocity moving towards positive offsets. However, at an angular offset range of $8^{\prime \prime}$ to $25^{\prime \prime}$ there is gas that has a decreasing velocity from $\sim 220 \mathrm{~km} \mathrm{~s}^{-1}$ to $190 \mathrm{~km} \mathrm{~s}^{-1}$ (the emission circled in the P-V diagram and located on the white segment of the red slice in Figure 49). The counter-rotating gas cloud could be a foreground/background gas cloud or counter-rotating gas in the disk. It has a large velocity range just below the velocities of the surrounding separate pieces of emission also seen in the VLA map. The counter rotating gas in 

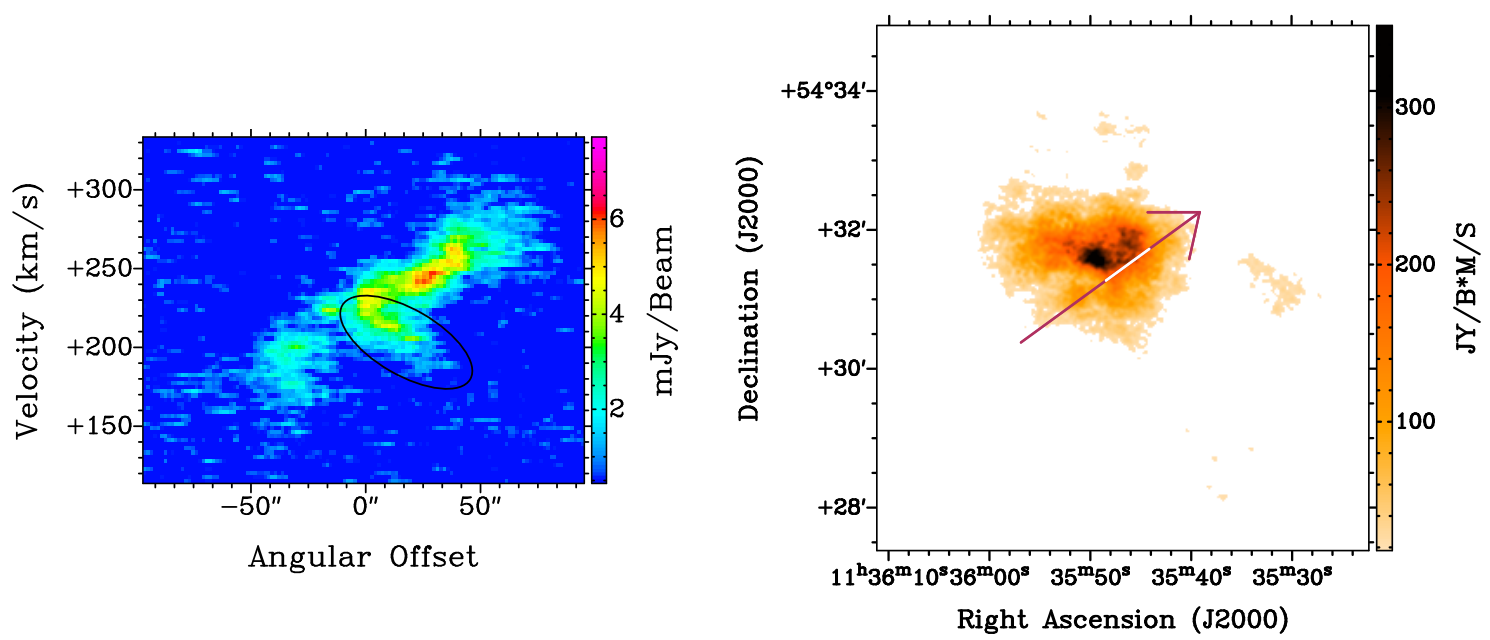

Fig. 49.- NGC 3738: The left column contains the P-V diagram and the right column contains the natural-weighted integrated H I map with a red arrow indicating the location of the corresponding slice through the galaxy and pointing in the direction of positive offset. This slice shows a counter rotating gas cloud found in the line of sight of NGC 3738's body. The location of the counter-rotating gas is indicated on the red arrow by a white line and circled in the P-V diagram.

Figure 49 cannot however account for the high velocity dispersions seen in the northeast side of the galaxy.

It is unclear what has happened to leave gas clouds surrounding NGC 3738, a counter-rotating gas cloud in the line of sight of NGC 3738's main body, and high velocity dispersions throughout the H I disk of NGC 3738. 14\% of the VLA H I mass lies outside of NGC 3738's disk and the VLA only recovered $56 \%$ of the mass recovered by the GBT. This indicates that a lot of emission may lie outside of the disk seen in NGC 3738's VLA H I maps. The excess emission could be from NGC 3738 or another source. The other source could be a galaxy or a large gas cloud that ran into NGC 3738, which left behind gas in the line of sight. However, NGC 3738 does not appear to have an extended, tenuous outer disk in the VLA data like those seen in the other BCDs in this dissertation, the surrounding gas clouds in the VLA data have velocities close to the systemic velocity of NGC 3738, and NGC 3738's stellar disk extends beyond the natural weighted H I 
emission. This could be evidence that the gas surrounding NGC 3738 once belonged to it. Also, the stellar morphological major axis as measured by Hunter \& Elmegreen (2006) is $-0.4^{\circ}$, which is offset from the kinematic major axis, measured to be about $120^{\circ}\left(-60^{\circ}\right.$; estimated by eye). A gas cloud alone running into IC 10 would not likely have enough force to explain the major axes' offset unless the gas cloud was of particularly high mass and/or moving at a large speed. The high velocity dispersions of the VLA H I emission also appear to indicate that the gas in the disk is quite disturbed. With loss of gas and high velocity dispersions, ram pressure stripping could be taking place in NGC 3738; however, NGC 3738 is in the Canes Venetici I group of galaxies. In such a loosely gravitationally bound group, it is unlikely that there is enough IGM to strip NGC 3738 of its gas. With no companion nearby and no obvious signatures of a merger, it is difficult to discern what has happened to NGC 3738. It is possible that it ran into another galaxy which has been left stripped of most of its gas. Deep stellar observations of the surrounding area could be used to look for a stellar companion nearby which may have lost its gas to NGC 3738 . 


\section{Conclusion}

The six BCDs in the LITTLE THINGS sample all appear to have kinematic and morphological disturbances. Each BCD appears to have different disturbances and different reasons for these disturbances:

- Haro 29's VLA H I column density has a curved, tenuous outer morphology that is rotating on a different kinematic axis than the dense inner region, which is rotating like a solid body. There are no companions near Haro 29 with which it may have interacted, as seen in the GBT H I column density maps (within the sensitivity of the maps). The outer tenuous gas may be gas that has recently been obtained by Haro 29, but if that is the case, it would be a large intergalactic gas cloud or several intergalactic gas clouds that Haro 29 has acquired. Haro 29 may also be an advanced merger that has had enough time for the dense inner $\mathrm{H}$ I region to settle into a regular rotation pattern, but not the outer tenuous gas.

- Haro 36 is a galaxy with a likely H I tidal tail. Tidal tails are signatures of interactions or mergers, however, there are no nearby companions with which it may have interacted seen in the GBT H I column density maps (within the sensitivity of the maps). The lack of companions near Haro 36 indicates that Haro 36 may instead be a merger. The stellar kinematics of this galaxy should be observed to look for signatures of a merger such as counter rotation and velocity dispersions twice the rotational velocity.

- Mrk 178 is a galaxy with a likely hole and possible shell structure that appears to dominate the morphology of most of the disk. There is also an extension of gas to the northwest that has a kinematic major axis nearly perpendicular to the kinematic major axis of the rest of the gas in Mrk 178. The northwest extension is also seen in the GBT data. The northwest extension does not appear to be tidal, from ram 
pressure stripping, or outflow from the hole. It is possible that it is a gas cloud that is being acquired by Mrk 178, but the low velocity dispersions may be indicating that this is a foreground/background gas cloud.

- VII Zw 403 is a galaxy that has a disturbed velocity field in the western side of its disk. The velocity disturbance is likely from a gas cloud in the foreground of the west side of the disk, which is disturbing the velocity field along the line of sight. The gas cloud may be outflow from a stalled hole just north of the densest $\mathrm{H} \mathrm{I}$ in the galaxy. It could also be a gas cloud falling onto VII Zw 403's disk. The remaining disk under the gas cloud has a kinematic major axis of with a PA that is offset by $\sim 55^{\circ}$ from the $\mathrm{H}$ I morphological axis and $\sim 66^{\circ}$ from the stellar morphological axis. This offset in major axes indicates that VII Zw 403 has likely experienced a external disturbance in its past.

- IC 10 is a galaxy with a complex morphology and velocity field. A new feature has been discovered in the GBT H I data of this galaxy; a northern H I extension. The northern extension could be a IGM gas filament that is being accreted onto IC 10 with increasing velocities in the plane of view. The northern extension is not likely a bridge, where the end of the northern extension is a companion galaxy to IC 10 , since there is no visible tidal tail associated with the potential companion galaxy. Another possibility is that the northern extension is a tidal tail and that IC 10 is an advanced merger. A potential remnant of a second galaxy can be seen in the GBT data to the east of the VLA data main body. The northern extension also has a turn around in velocity which would indicate, that if it is a tidal tail, then it may be beginning to fall back into the galaxy. Higher resolution $\mathrm{H}$ I images of the northern extension and its connection to IC 10's main body, along with deep optical images of the two potential locations for a companion galaxy are necessary to help determine what has happened to IC 10. 
- NGC 3738 is surrounded by small H I clouds in the VLA data. It also has counter-rotating gas in the line of sight of its disk. The mass of the emission outside of the main disk in the VLA data appears to be significant, with 14\% of the VLA measured mass being outside of the main disk and the VLA only detecting $56 \%$ of the mass seen in the GBT. It is therefore possible that NGC 3738 has a significant amount of tenuous gas surrounding it. The VLA H I data suggest that something may have run into NGC 3738, but with no known objects nearby, and no H I companion seen in the GBT data, it is unclear as to what could have disturbed NGC 3738 .

Each BCD in this sample has likely experienced an external disturbance. Potential dwarf-dwarf mergers in the sample include: Haro 29, Haro 36, and IC 10. If Haro 29 is a dwarf-dwarf merger, then its tidal tails may have already dissipated or may be less prominent depending on how the merger took place. For example, retrograde interactions create very small tidal features (Toomre \& Toomre 1972). If Haro 29 is a dwarf-dwarf merger that once had prominent tidal tails, then it is likely a more advanced merger than IC 10 and Haro 36, which still have prominent potential tidal tails. BCDs potentially consuming intergalactic gas include: Haro 29, Mrk 178, VII Zw 403, and IC 10. VII $\mathrm{Zw}$ 403's underlying $\mathrm{H}$ I disk has an offset kinematic axis from the $\mathrm{H} \mathrm{I}$ and stellar morphological axis and had a peak in star formation several hundred Myr ago. Therefore, if it is consuming intergalactic gas, then it may have also done so in the past to fuel its star formation. Mrk 178 has also had a recent increase in star formation which appears to have blow a hole in the disk. Its increased star formation in the past Gyr may also have been fueled by past gas accretion.

With such a small sample it is not possible to extrapolate and make statements about $\mathrm{BCDs}$ as a whole. However, within this sample, it is clear that these BCDs that were thought to be isolated are not likely to have evolved in isolation. Even the galaxies in the 
low galaxy density Canes Venetici I group of galaxies are not evolving in isolation. Haro 29, Mrk 178, and NGC 3738 are all in this loosely gravitationally bound group of galaxies and all appear isolated with respect to other galaxies. Yet, Mrk 178 still appears to have a large gas cloud nearby that is probably in the foreground or background of the galaxy. Haro 29 is a likely merger or may have accreted several large gas clouds that surrounded it. NGC 3738's data show a significant amount of evidence for an external disturbance. Therefore, even in a loosely gravitationally bound, low galaxy density group like the Canes Venetici I group of galaxies, there is still evidence for external disturbance in these galaxies. This indicates that the space surrounding these galaxies is not currently or has not always been empty.

Each of these six BCDs appears to have different H I morphologies and kinematics. However, the overall theme seen in each BCD is a disturbed H I morphology and disturbed H I kinematics. The H I data suggests that in each case, a large external disturbance such as a dwarf-dwarf merger or consumption of external gas is needed in order to create the H I structure seen today. This could be indicating that even in the most isolated BCDs, the starburst may have been triggered through gaining fuel from outside sources (IGM or another galaxy). 


\section{List of References}

Ashley, T., Simpson, C. E., \& Elmegreen, B. G. 2013, AJ, 146, 42

Banerjee, A., Jog, C. J., Brinks, E., \& Bagetakos, I. 2011, MNRAS, 415, 687

Barnes, J. E. 1988, ApJ, 331, 699

Begum, A., Chengalur, J. N., Karachentsev, I. D., Sharina, M. E., \& Kaisin, S. S. 2008, MNRAS, 386, 1667

Bekki, K. 2008, MNRAS, 388, L10

Benson, A. J. 2010, PhR, 495, 33

Braatz, J. 2009, Calibration of GBT Spectral Line Data in GBT IDL v2.1, http://www.gb.nrao.edu/GBT/DA/gbtidl/gbtidl_calibration.pdf

Brinchmann, J., Kunth, D., \& Durret, F. 2008, 485, 657

Brooks, A. M., Governato, F., Quinn, T., Brook, C. B., \& Wadsley, J. 2009, ApJ, 694, 396

Brunthaler, A., Reid, M. J., Falcke, H., Henkel, C., \& Menten, K. M. 2007, A\&A, 462, 101

Chevalier, R. A. 1974, ApJ, 188, 501

Chynoweth, K., Holley-Bockelmann, K., Polisensky, E., \& Langston, G. 2011, AJ, 142, 137

Cornwell, T. J. 2008, IEEE Journal of Selected Topics in Signal Processing, 2, 793

Cox, T. J., Jonsson, P., Somerville, R. S., Primack, J. R., \& Dekel, A. 2008, MNRAS, 348,386

Dale, D. A., Cohen, S. A., Johnson, L. C., et al. 2009, ApJ, 703, 517

de Blok, W. J. G. Walter, F., Brinks, E. et al. 2008, AJ, 136, 2648

Gil de Paz, A. \& Madore, B. F. 2005, ApJS, 156, 345 
Gonçlaves, D. R, Teodorescu, A. M, Alves-Brito, A., Méndez, R. H., \& Magrini, L. 2012, MNRAS, 425, 2557

González-Riestra, R., Rego, M., \& Zamorano, J. 1988, A\&A, 202, 27

Guseva, N. G., Izotov, Y. I., \& Thuan, T. X. 2000, ApJ, 531, 776

Högbom, J. A. 1974, ApJS, 15, 417

Hunter, D. A. \& Elmegreen, B. G. 2004, AJ, 128, 2170

Hunter, D. A. \& Elmegreen, B. G. 2006, ApJS, 162, 49

Hunter, D. A., Elmegreen, B. G., \& Ludka, B. C. 2010, AJ, 139, 447

Hunter, D. A., Ficut-Vicas, D., Ashley, T. et al. 2012, AJ, 144, 134

Huchtmeier, W. K. 1979, A\&A, 75, 170

Ibata, R. A., Lewis, G. F., Conn, A. R., et al. 2013, Nature, 493, 62

Izotov, Y. I., Thuan, T. X., \& Lipovetsky, V. A. 1997, ApJS, 108, 1

Jarrett, T. H., Chester, T., Cutri, R., Schneider, S. E., \& Huchra, J. P. 2003, AJ, 125, 525

Kaisin, S. S. \& Karachentsev, I. D. 2008, A\&A, 479, 603

Karachentsev, I. D., Dolphin, A. E., Grisler, D., et al.2002, A\&A, 383, 125

Karachentsev, I. D., Sharina, M. E., Dolphin, A. E., et al.2003, A\&A, 398, 467

Kehrig, Pérez-Montero, E., Vílchez, J. M., Brinchmann, J., et al. 2013, MNRAS, 432, 2731

Kennicutt, R. C., Jr., Lee, J. C., Funes, J. G., Sakai, S., \& Akiyama, S. 2008, ApJS, 178, 247

Kereš, D., Katz, N., Weinberg, D. H., \&, Davé 2005, MNRAS, 363, 2

Lee, J. C., Gil de Paz, A., Kennicutt, R. C., Jr. et al. 2011, ApJS, 192, 6 
Lelli, F. 2013, PhD Thesis, Starbursts and Gas Dynamics in Low-Mass Galaxies, http://www.astro.rug.nl/ lelli/Lelli.PhDthesis.pdf

Leroy, A. K., Walter, F., Brinks, E., et al. 2008, AJ, 136, 2782

Lira, P., Lawrence, A., \& Johnson, R. A. 2000, MNRAS, 319, 17

Lynds, R., Tolstoy, E., O’Niel, E. J. Jr., Hunter, D. A. 1998, AJ, 116, 146

Mac Low, M.-M. \& Ferrara, A. 1999, ApJ, 513, 142

Manthey, E., \& Oosterloo, T. 2008, The Evolution of Galaxies Through the Neutral Hydrogen Window, 1035, 156

Marlowe, A. T., Heckman, T. M., Wyse, R. F. G., \& Schommer, R. 1995, ApJ, 438, 563

Martínez-Delgado, D., Romanowsky, A. J., Gabany, R. J. et al. 2012, ApJ, 784, L24

Massey, P. \& Armandroff T. E. 1995, AJ, 109, 2470

Mazzarella, J. M., Bothun, G. D., \& Boroson, T. A. 1991, AJ, 101, 2034

McCray, R. \& Kafatos, M. 1987, ApJ, 317, 190

Mihos, J. C. 2004, IAU Symposium Series, The Evolution of Tidal Debris, 217, 390

Mihos, J. C. \& Hernquist, L. 1996, ApJ, 464, 641

Nicholls, D. C., Dopita, M. A., Jerjen, H, \& Meurer, G. R.2011, AJ, 142, 83

Nidever, D. L., Ashley, T., Slater, C. T., et al.. 2013, ApJ, 779, L15

Noeske, K. G., Iglesias-Páramo, J., Vílchez, J. M., Papaderos, P., \& Fricke, K. J. 2001, A\&A, 371, 806

Noguchi, M. 1988a, A\&A, 201, 37

Noguchi, M. 1988b, A\&A, 203, 259

Ott, J., Walter, F., \& Brinks, E. 2005, MNRAS, 358, 1423 
Papaderos, P., Fricke, K. J., Thuan, T. X., \& Loose, H.-H. 1994, A\&A, 291, L13

Papaderos, P., Loose, H.-H., Thuan, T. X, \& Fricke, K. J. 1996, A\&AS, 120, 207

Perley, R. A., Chandler, C. J., \& Wrobel, J. M. 2011, ApJ, 739, L1

Paturel, G., Theureau, G., Bottinelli, L., et al.2003, A\&A, 412, 57

Peterson, S. D. 1979, ApJS, 40, 527

Pustilnik, S. A., Kniazev, A. Y., Lipovetsky, V. A., \& Ugryumov, A. V. 2001, A\&A, 373, 24

Richer, M. G., Bullejos, A., Borissova, J. et al.2001, A\&A, 370, 34

Roychowdhury, S., Chengalur, J. N., Begum, A., \& Karachentsev, I. D. 2009, MNRAS, 397, 1435

Sancisi, R., Fraternali, F., Osterloo, T., \& van der Hulst, T. 2008, A\&A Rev., 15, 189

Sanna, N., Bono, G., Stetson, P. B., et al. 2010, ApJ, 722, L244

Schulte-Ladbeck, R. E., \& Hopp, U.1998, AJ, 116, 2886

Schulte-Ladbeck, R. E., Hopp, U., Greggio, L., \& Crone, M. M. 2000, AJ, 120, 1713

Schulte-Ladbeck, R. E., Hopp, U., Greggio, L., Crone, M. M., \& Drozdovsky, I. O. 2001, AJ, 121, 3007

Schwartz, C. M., Martin, C. L., Chandar, R., et al. 2006, ApJ, 646, 858

Shostak, G. S., \& Skillman, E. D. 1989, A\&A, 214, 33

Simpson, C. E. \& Gottesman, S. T. 2000, AJ, 120, 2975

Simpson, C. E., Hunter, D. A., Nordgren, T. E., et al. 2011, AJ, 142, 82

Stevens, I. R. \& Strickland, D. K. 1998, 294, 523

Stil, J. M. \& Israel, F. P. 2002, A\&A, 389, 29 
Tajiri, Y. Y. \& Kamaya, H. 2002, A\&A, 389, 367

Tamburro, D., Rix, H.-W., Leroy, A. K. et al. 2009, AJ, 137, 4424

Taylor, C. L. 1997, ApJ, 480, 524s

Taylor, C. L., Brinks, E., Grashuis, R. M., \& Skillman, E. D. 1995, ApJS, 99, 427

Thuan, T. X. \& Martin, G. E. 1981, ApJ, 247, 823

Thuan, T. X., Hibbard, J. E., \& Lévrier, F. 2004, AJ, 128, 617

Thilker, D. A., Braun, R., Walterbos, R. A. M., et al. 2004, ApJ, 601, L39

Toomre, A. \& Toomre, J. 1972, ApJ, 178, 623

Tully, R. B., Boesgaard, A. M., Dyck, H. M., \& Schempp 1981, ApJ, 246, 38

Tully, R. B. 2013, Nature, 493, 31

Vaduvescu, O., Richer, M. G., \& McCall, M. L. 2006, 131, 1318

van Zee, L., Salzer, J. J., \& Skillman, E. D. 2001, AJ, 122, 121

Viallefond, F. \& Thuan, T. X. 1983, ApJ, 269, 444

Warren, S. R., Skillman, E. D., Stilp, A. M. et al. 2012, ApJ, 757, 84

Westmeier, T., Brüns, C., \& Kerp, J. 2008, MNRAS, 390, 1691

Wilcots, E. M. \& Miller B. W. 1998, AJ, 116, 2363

Yin, J., Magrini, L., Matteucci, F., Lanfranchi, G. A., Gonçlaves, D. R, \& Costa, R. D. D. 2010, A\&A, 520, 10 


\section{Appendices}

\section{The Size of Radio Telescopes}

Radio telescopes are mirrors for the radio regime of the spectrum. For telescopes, angular resolution $\sim \lambda / \mathrm{D}$, where $\lambda$ is the wavelength being observed and $\mathrm{D}$ is the diameter of the telescope. Radio wavelengths are long (radio wavelength are $1 \mathrm{~mm}-100 \mathrm{~km}$ while optical wavelengths are on the order of $10^{-9} \mathrm{~m}$ ) and therefore radio telescopes require large diameters to obtain data of reasonably good angular resolution. There are two ways to deal with the large diameters necessary to obtain reasonable angular resolution in radio telescopes: one is to build very large single dish telescopes and the other is to build interferometers (discussed in Appendix 2). The size of large, single dish telescopes can quickly become troublesome structurally and scientifically; the telescope must be built to not only withstand weather, but also to withstand its own weight. Depending on the position of the telescope, gravity will deform the dish to different shapes which must be accounted for in data collection. There have been technical feats in making large single dish radio telescopes, but even the largest fully steerable telescope in the world, the GBT, only has an angular resolution of $\sim 9^{\prime}$ for $\mathrm{H}$ I observations. So although these large single dish telescopes have high sensitivity, they are not able to resolve objects that are angularly small. Interferometers have been used to overcome this problem.

\section{Interferometry}

Radio interferometry is a technique that allows the combination of signals from many antennae to work together as one large telescope. Each antenna pair creates a baseline pair where a baseline (not the same baseline as mentioned in the data reduction 
techniques of the GBT data) is the distance between the two antenna. Together, all of the antennae synthesize a large radio telescope with a diameter the size of the longest baseline. Interferometers are a unique solution to the problem of a radio telescope dish needing to be large to obtain a reasonable angular resolution (see the discussion of radio telescope sizes in Appendix 1) in that each dish can be built to be structurally sound (not too large) but all of the dishes can then work together to synthesize as large of a telescope as is desired.

Interferometers however, do not have the benefit of the filled spacing in between the individual dishes as single dish telescopes do. The earth's rotation helps to account for some of this loss because the configuration of the dishes, although stationary on Earth, will appear, to the object that is being observed, to be rotating as Earth rotates. This rotation acts as a way for interferometers to synthesize a more filled dish. To see this, imagine two dishes in an interferometer that create a baseline that runs parallel to the lines of longitude on the earth. If there is an observer watching them from outside of the earth, they will appear to have the shortest distance between them when they are at the projected edges of the earth and the longest distance between them when they are at the projected center of their line of longitude. This can be seen in Figure 50, where two antennae (the small blue and purple circles) are drawn rotating on earth. The projected distance between the dishes is indicated by the white line. The distance appears larger when they are at the projected center of earth. These projection effects create new baseline lengths that can at least partially fill the synthesized dish. 


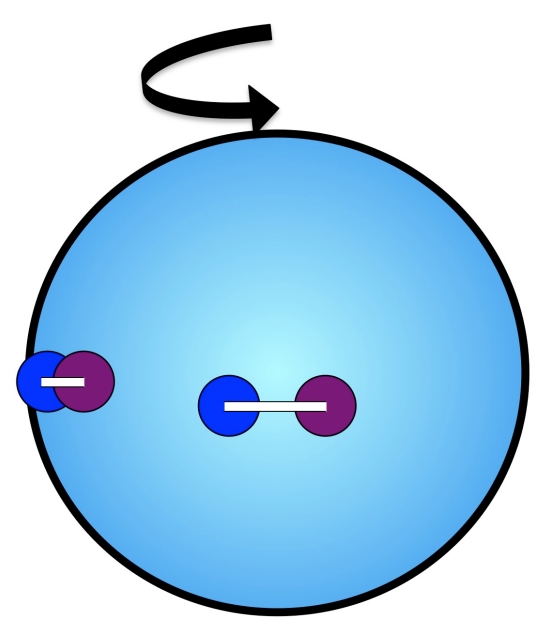

Fig. 50. - The Earth is represented by the largest circle. Two antennae, the blue and purple circles, have different baselines (the white line) as the earth rotates.

Table 6. VLA Configuration Information

\begin{tabular}{lccc}
\hline \hline Configuration & $\begin{array}{c}\text { Max. Baseline } \\
(\mathrm{km})\end{array}$ & $\begin{array}{c}\text { Beamwidth }^{\dagger} \\
(\operatorname{arcsec})\end{array}$ & $\begin{array}{c}\text { Max. Size of Feature } \\
\text { Detectable }(\operatorname{arcsec})\end{array}$ \\
\hline A & 1 & 1.3 & 36 \\
$\mathrm{~B}$ & 3.6 & 4.3 & 120 \\
$\mathrm{C}$ & 10 & 14 & 970 \\
$\mathrm{D}$ & 36 & 46 & 970 \\
\hline
\end{tabular}

${ }^{\dagger}$ These numbers are for observations taken at $21 \mathrm{~cm}$. 


\subsection{The VLA}

The VLA is an interferometer that consists of 27 antennas placed in a Y-shaped configuration. Each dish in the VLA is 25 meters in diameter. The dishes are configured into four main configuration s (which can then be altered as needed to account for objects at low elevations) named by letters A through D. Information about each configuration can be found in Table 6. The A-configuration is the largest configuration and thus has the smallest beam (for more information about beams, see Appendix 3 below), giving the A-configuration the highest resolution and lowest sensitivity. The D-configuration is the smallest synthesized telescope and thus has the largest beam, giving the D-configuration the lowest resolution and highest sensitivity.

\section{Spatial Resolving Power: "Beams"}

Radio telescopes collect information via antennae. Since Maxwell's equations of electro-magnetism can be written without any time dependence (Maxwell's equations give the same results if time is run backwards or forwards) antennas can then be thought of as receivers or transmitters mathematically. The results of our observations will therefore be the same if we think of the antenna as transmitting radiation to the source of interest or the radiation from the source of interest being received by the antenna. This concept gives rise to what radio astronomers call a beam.

A beam is the power pattern that a radio telescope will transmit if it is thought of as a transmitter. A telescope dish of larger diameter will produce a smaller beam size which will observe at a higher resolution and lower sensitivity than a dish of smaller diameter. For a radio telescope, the shape of the beam is important because you want to transmit the most power to the source of interest, not the surrounding area, but an antenna does not 
transmit a perfect collimated beam of radiation. Antennas that are using a dish to transmit power will typically have a main beam that has a three dimensional elongated tear drop shape and then several tear drop shaped side lobes. These side lobes are power patterns, typically with lower maximum transmitting power than the main beam, that do not point towards the region of interest. Sidelobes thus collect data from the ground if they point towards the ground or from regions of the sky that do not contain the object of interest. These side lobes are often reduced as much as possible in the construction of the telescope and their effects are then modeled for later removal in the data processing steps.

\section{Weighting Schemes}

Two of the most common weighting schemes used for mapping interferometry data are natural weighting and robust weighting. These two weighting schemes are used to map the data at different resolutions and sensitivities by giving weight to the data coming from each baseline pair, thus adjusting the synthesized beam size. Higher sensitivity and lower resolution maps are made with natural weighting; all of the baseline pairs will have the same weight. Since there are more short baselines in the configurations than there are long baselines (more pairs of dishes close together can be made than pairs of dishes far apart), the short baselines will end up with more total weight in the image, creating a larger synthesized beam. Robust weighting is essentially a way of tuning the amount of weighting that is given to longer baselines. The robust parameter can be set from -5 to 5 , where 5 is similar to natural weighting and -5 gives the most weight to the longer baselines. So a robust parameter of -5 creates a small synthesized beam, resulting in maps of high resolution and low sensitivity, this also increases the noise in maps. 


\section{Nyquist Sampling}

Nyquist Sampling is a mathematical theory that states that a wave must be sampled at a rate of at least twice the highest frequency contained within the wave in order to completely reconstruct the wave from the data obtained. To understand the reason for this, imagine a single frequency sinusoidal wave (the black wave in Figure 51) being sampled once every one and a half wavelengths, as shown by the blue circles in Figure 51. Assuming the simplest fit, you could then fit a sinusoidal wave to the data collected with wave that has a wavelength of 3 times the wavelength of the original data (the blue line in Figure 51). This would be an undersampled data set; higher frequency data could be interpreted as lower frequency data. This will alias spurious low frequency data into the resulting data, meaning that high frequency data is being mis-interpreted as low frequency data. If we sample according to Nyquist theory (at least twice the highest frequency or half a wavelength) as shown by the pink circles in Figure 51, we should not have any aliased signals if all fits are kept to their simplest form (fit given by the pink dotted line).

\section{Smoothing}

The Hanning smoothing done for the GBT data is a technique that uses three frequency channels of a spectrum at a time to smooth. Each pixel in the first channel (in velocity/frequency space) used will have its intensity value multiplied by one-fourth, the second channel will have its intensity value multiplied by one-half, and the third channel will have its intensity value multiplied by one-fourth. The adjusted values in the channels are then added together to create a new intensity value for the second channel. The smoothing function will then move over by only one channel, so that the original second channel is now the first channel and the original third channel is now the second channel. This smoothing function will only decrease the total number of channels by two and keep 


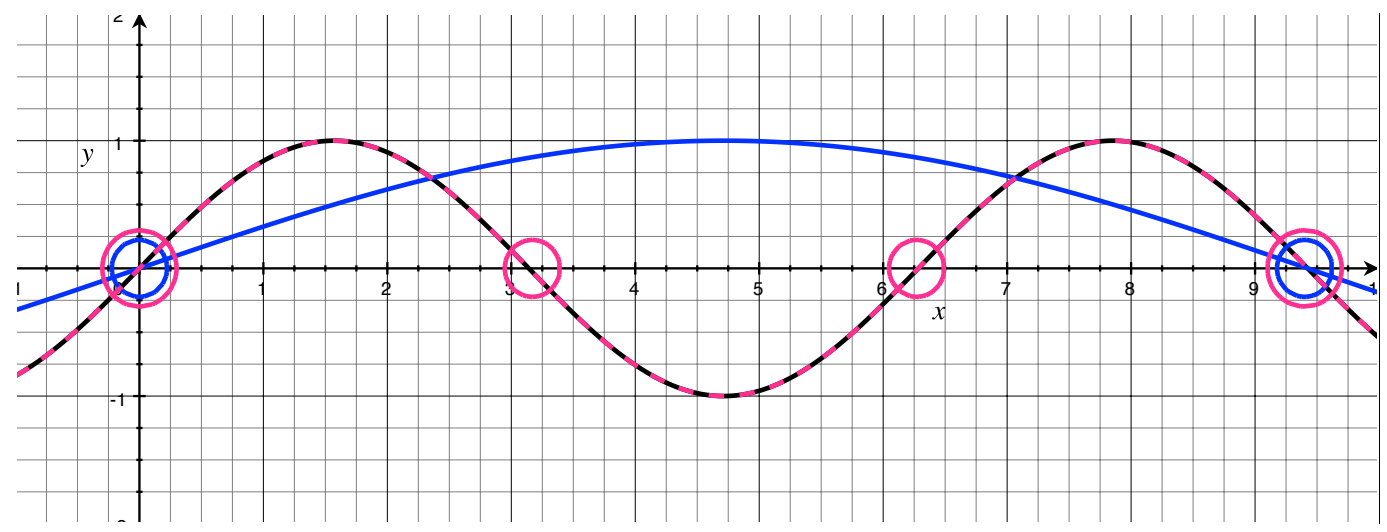

Fig. 51.- Sine wave sampling rate example, where the pink circles represent Nyquist sampling and the blue circles represent undersampling. The simplest fits to these sampling rates are shown in the corresponding colors of the circles and the black sine wave represents the original sine wave.

the same velocity resolution (one channel from each end of the spectrum is lost since the first and last channels are never replaced by a value).

The boxcar smoothing done for the GBT data is smoothing by a simple average of a given number of channels. The number of channels to be averaged is set by the user. By using this smoothing function the number of channels is decreased: number $_{\mathrm{nc}}=\left\lfloor\right.$ number $_{\mathrm{oc}} /$ number $\left._{\text {boxcar }}\right\rfloor$, where number ${ }_{\mathrm{nc}}$ is the number of new channels, number $_{\mathrm{oc}}$ is the number of original channels before smoothing, and number boxcar $_{\text {is }}$ the number of channels that will be averaged in the boxcar function. For example, a boxcar 
averaging of 3 is done by averaging three channels at a time and replacing all three channels with a single channel. Therefore if you have 180 channels prior to smoothing, you would be left with 60 channels after smoothing. Your velocity resolution in this case will also be three times lower since three velocity channels were averaged together.

\section{NGC 3738's baseline fitting}

Two sources were located outside of the target frequency range in NGC 3738's data and with calibration, have been reflected into NGC 3738's target frequency range. The real emission from these sources is very far away from NGC 3738 in velocity space (about $1000 \mathrm{~km} \mathrm{~s}^{-1}$ ). It is therefore very unlikely that these sources are interacting with NGC 3738.

One of these sources is located to the bottom-right of NGC 3738 in the GBT maps shown in Figure 47. The spectra of the approximate space that it covered were summed and averaged to get a clear picture of where it is in frequency space; the resulting labeled spectrum can be seen in Figure 52. This spectrum has not had a baseline fit to it or the RFI removed from it, which is why the spectrum appears to have no zero line of emission and there are also spikes at $\sim 1.414 \mathrm{MHz}$ and $\sim 1.420 \mathrm{MHz}$. The Milky Way and its reflection have been labeled as such. The source with emission located at $1414.3-1415.3 \mathrm{MHz}$ is the source that has been reflected into the target frequency range at 1417.8-1418.8 MHz. The frequency range that this source occupies in the target frequency range can now no longer be used as zero emission space in a baseline fit. If it is used or partially used, then this can result in a fake source at its location and generally poor fits since a low order polynomial is being fit to the baseline. An example of a fit using the frequency range that includes the reflections of these sources can be seen in the top of Figure 52. A second example of a 


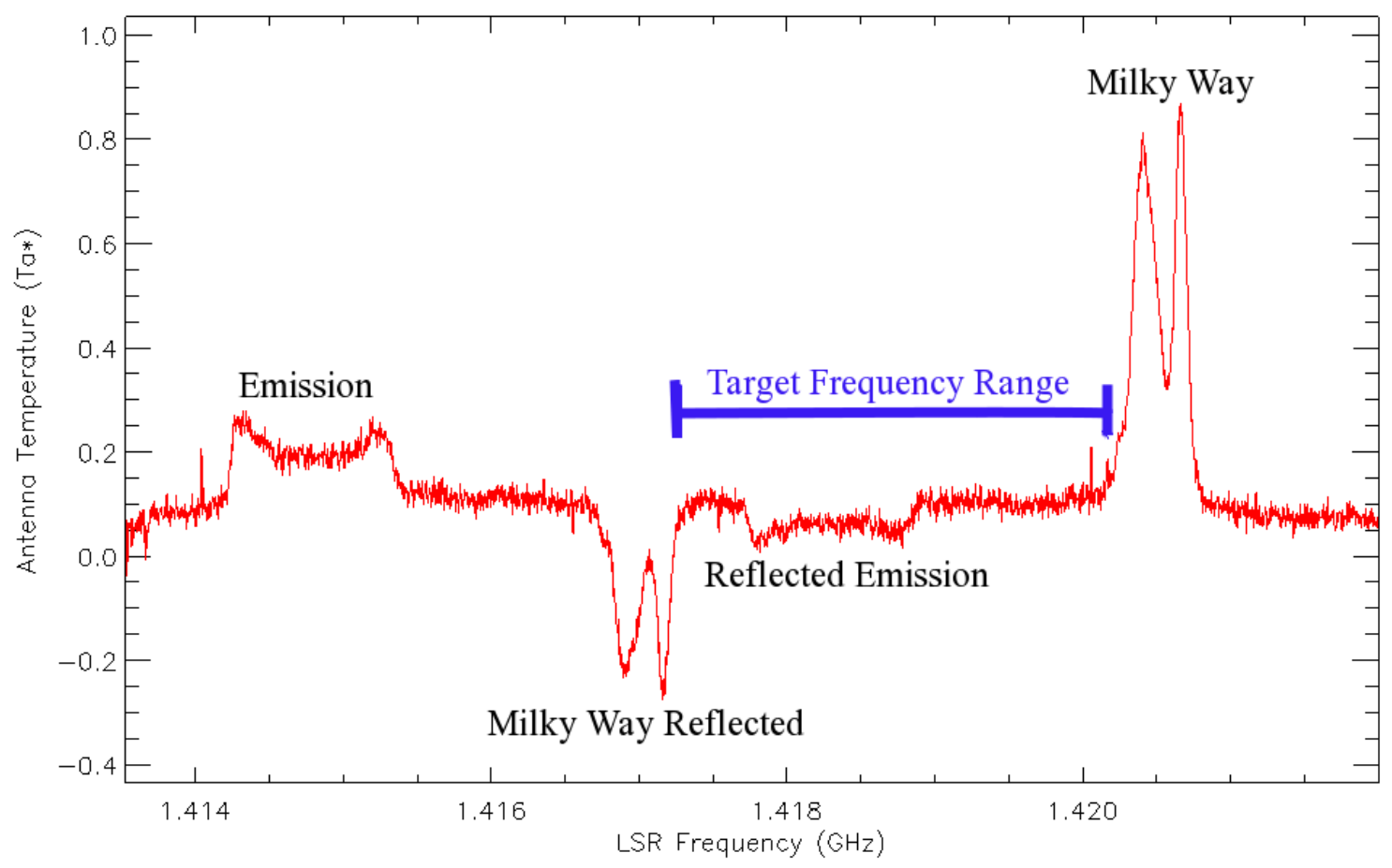

Fig. 52.- The emission from a source that was reflected into NGC 3738's GBT target frequency range.

fit using frequency ranges that do not overlap with the reflected source can be seen in the bottom of Figure 52.

An averaged spectrum of NGC 3738's emission is shown in Figure 54. In this spectrum, NGC 3738 peaks at a frequency of $1419.3 \mathrm{MHz}$, and its reflection appears at a frequency of 1415.8 MHz. The reflection now occurs outside of the target frequency range. The emission of NGC 3738 and the reflection of the other source now consume most of the target frequency range, leaving very little room to fit to a baseline. Some of the emission and reflection-free space outside of the target frequency range can be used for baseline fitting when the baseline appears continuous, but using a significant portion of the target frequency range is necessary for a good fit. There is also the added problem of another source in the maps of NGC 3738 being reflected into the target frequency range. In the 

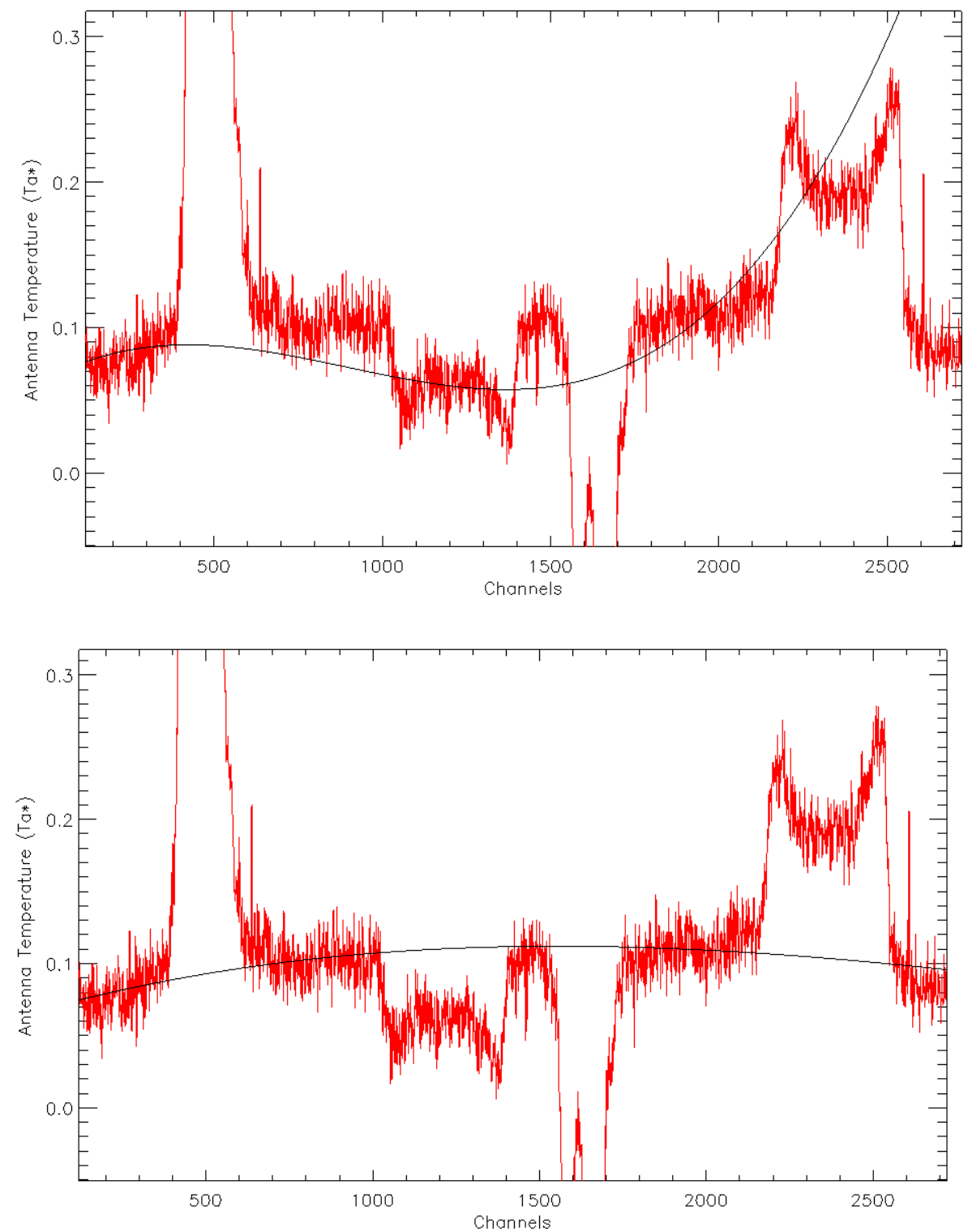

Fig. 53. - Top: An example of a bad baseline fit using channels (0-250; 674-720; 10701380 ; 1820-2062). Bottom: An example of a good baseline fit using channels (0-250; 674900; 1820-2062).

GBT maps, a third, faint source appears just above (southwest) NGC 3738. Its averaged spectrum is shown in Figure 55. In this figure, the top source has emission at $1415 \mathrm{MHz}$ and has a reflection at $1418.5 \mathrm{MHz}$. 
With the two reflected sources and NGC 3738 on the GBT maps stretching throughout the target frequency range, it is impossible to fit one single baseline to all three sources. Luckily, the two reflected sources and NGC 3738 are all separated in Galactic Latitude. Therefore, the solution to the baseline problem was to split the GBT map into four spatial regions, with different baseline fit parameters for each region. The first region included the raster scan rows (in Galactic Latitude) that contained only the first reflected source. The second region included only the raster scan rows that contained NGC 3738. The third region included the reflected source above NGC 3738. The fourth region included everything else. The baseline fit used for NGC 3738 was also used for this fourth region so that any sources close to NGC 3738 in velocity could be fit properly. The rows with the reflected sources also had their emission free pixels searched for any possible emission that could be related to NGC 3738 or a companion of NGC 3738 's. This was done by averaging 10 spectra at a time (the approximate number of spectra that made up each of the sources in the map) to look for possible emission in the resulting averaged spectrum. The exceptions to this 10 spectra averaging were the spectra close to the reflected sources and the last four spectra of each row, where fewer spectra were available for averaging, but still averaged for inspection. No other sources were found in the target frequency range. This method gives the four different regions slightly different noise levels, but the effect is small. 


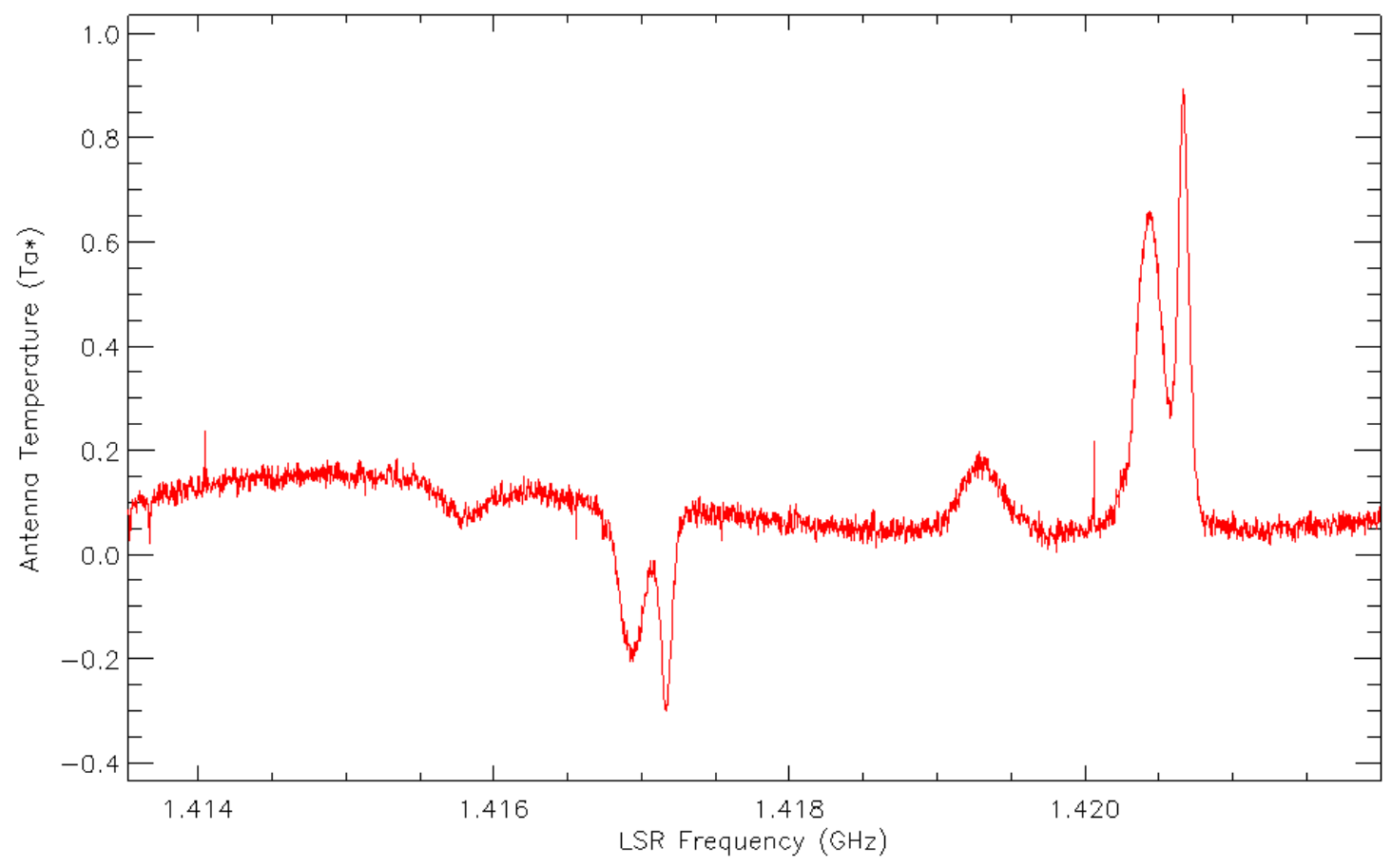

Fig. 54. - The averaged spectra of NGC 3738 from the GBT data. 


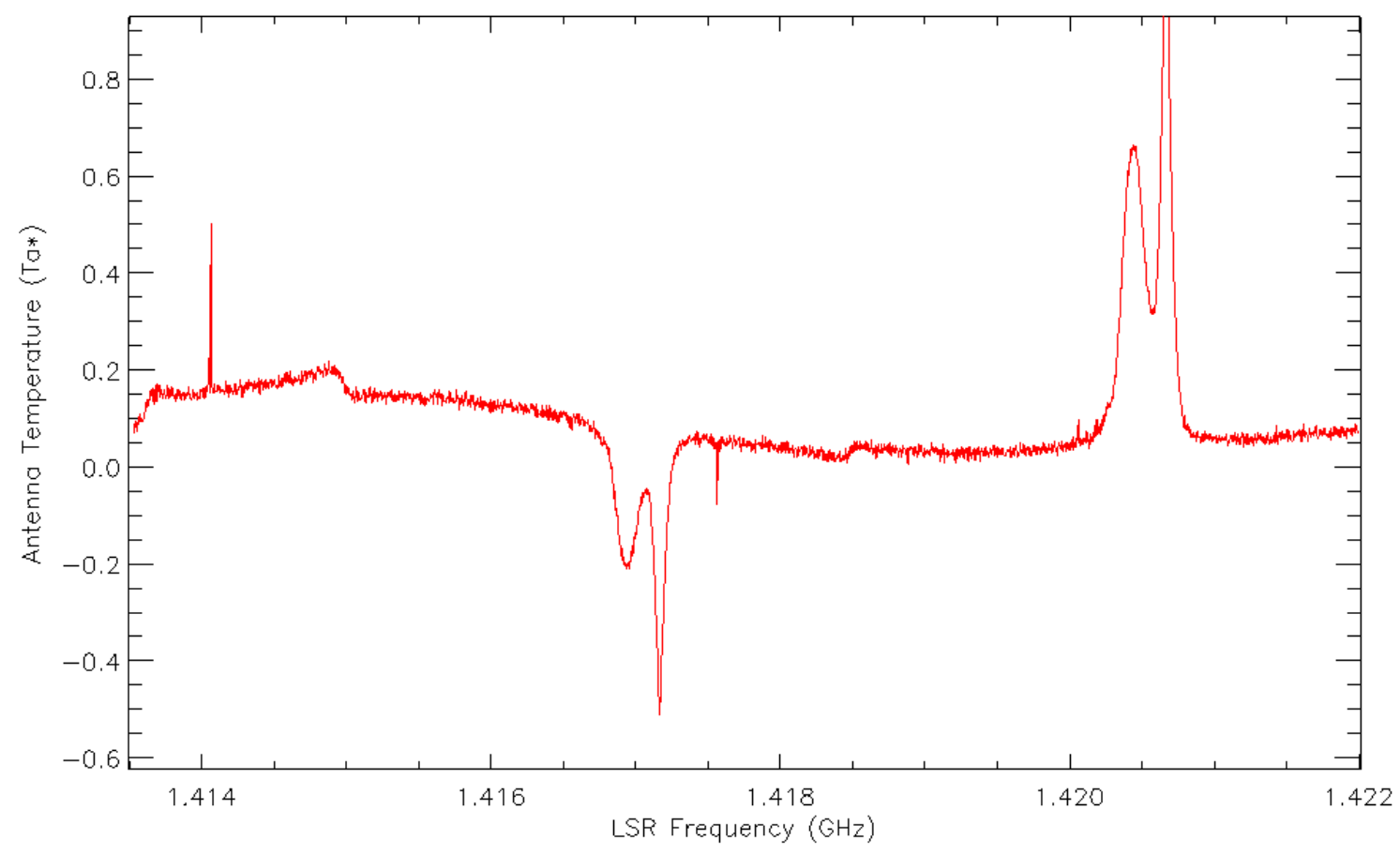

Fig. 55.- The averaged spectra of a second galaxy that is reflected into the target frequency range of NGC 3738 . 
VITA

\section{TRISHA ASHLEY}

Born, Rochester, New York

2004-2008 Bryn Mawr College: Physics Tutor, Physics Peer-Led

Instructor, Physics Laboratory Teaching Assistant

Summer 2007 SARA Research Experience for Undergraduates (REU) program, mentored by Caroline Simpson, FIU

2008

A.B. in Physics and Astronomy

Bryn Mawr College, Bryn Mawr, PA

2008-2013 FIU: Graduate Research Assistant, Teaching Assistant

2013-2014 FIU Dissertation Year Fellowship

April 2013 First Place: Graduate Student Research Competition, FIU Physics Department

March 2014 Second Place: GSAW Scholarly Forum, FIU

\section{PUBLICATIONS AND PRESENTATIONS}

Ashley, T., "External Perturbations in the Blue Compact Dwarf Galaxy Haro 36,"

Research Competition, Department of Physics, Florida International University, May 2013

Ashley, T., "External Perturbations in the Blue Compact Dwarf Galaxy Haro 36" (modified to include new results), Galactic Gas Supply Workshop, Green Bank Observatory, Green Bank, WV, May 2013

Ashley, T., “The H I Chronicles of LITTLE THINGS BCDs,” FIU Graduate Student Appreciation Week Scholarly Forum, Florida International University, March 2014

Ashley, T. \& Simpson, C. E., "VII Zw 403, Structure Resolved in a Blue Compact Dwarf Galaxy," 2008, Journal of the Southeastern Association for Research in Astronomy, 2, 30 (5pp)

Ashley, T. \& Simpson, C. E., “VII Zw 403, Structure Resolved in a Blue Compact Dwarf Galaxy,” 2008, American Astronomical Society Meeting Abstracts, 211, \#13.10; Bulletin of the American Astronomical Society, Volume 39, 756

Ashley, T. \& Simpson, C. E., “The H I Chronicles of LITTLE THINGS BCDs,” AAS Meeting, Washington, D.C., January 2014 
Ashley, T., Simpson, C. E., \& Elmegreen, B. G., "The H I Chronicles of LITTLE THINGS BCDs: Evidence for External Perturbations in the Morphology and Kinematics of Haro 29 and Haro 36," 2013, The Astronomical Journal, 146, 42 (17pp)

Basu, S., Ashley, T., Simpson, C. E., Hunter, D. A., \& the LITTLE THINGS Team, "The LITTLE THINGS Survey,” 2009, Assembly, Gas Content and Star Formation History of Galaxies, The Fourth North American ALMA Science Center Conference, http://www.cv.nrao.edu/php/meetings/galaxies09/posterabstracts.php

Hunter, D. A., Ficut-Vicas, D., Ashley, T., Brinks, E., Cigan, P., Elmegreen, B. G., Heesen, V., Herrmann, K. A., Johnson, M., Oh, S.-H., Rupen, M. P., Schruba, A., Simpson, C. E., Walter, F., Westpfahl, D. J., Young, L. M., \& Zhang, H.-X., "LITTLE THINGS," 2012, The Astronomical Journal, 144, 134 (29pp)

Hunter, D. A. \& the LITTLE THINGS Team, “The LITTLE THINGS Survey,” 2012, American Astronomical Society Meeting Abstracts, 219, \#148.01

Nidever, D. L., Ashley, T., Slater, C. T., Ott, J., Johnson, M., Bell, E. F., Stanimirović, S., Putman, M., Majewski, S. R., Simpson, C. E., Burton, W. B., Juette, E., \& Oosterloo, T., "Evidence for an Interaction in the Nearest Starbursting Dwarf Irregular Galaxy IC 10," 2013, The Astrophysical Journal, 779, L15 (6pp)

Simpson, C. E., Hunter, D. A., \& Ashley, T., “VII Zw 403: A Blue Compact Dwarf Case Study," 2008, Proceedings of the International Astronomical Union, Volume 4, Symposium S255, DOI: http://dx.doi.org/10.1017/S1743921308025155

Simpson, C. E., Hunter, D. A., Nordgren, T. E., Brinks, E., Elmegreen, B. G., Ashley, T., Lynds, R., McIntyre, V. J., O’Neil, E. J., Östlin, G., Westpfahl, D. J., \& Wilcots, E. M., "VII Zw 403: H I Structure in a Blue Compact Dwarf Galaxy," 2011, The Astronomical Journal, 142, 82 (21pp) 